\title{
Passenger Acceptance of Alignments with Frequent Curves in Maglev or Other Very-High-Speed Ground Systems
}

\author{
10/31/95 12:58:30 PM
}

John K. Pollard

Leonore Katz-Rhoads

Peter Mengert

Robert DiSario

E. Donald Sussman

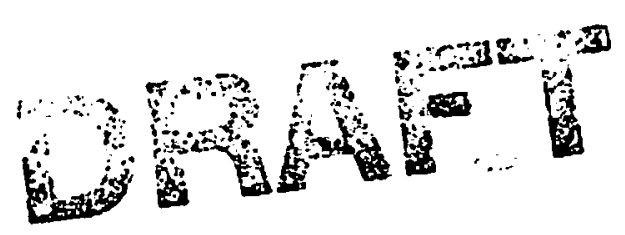




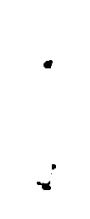

$-$ 


\section{PREFACE}

This report was prepared by the Operator Performance and Safety Analysis Division of the Office of Research and Analysis at the Volpe National Transportation Systems Center (VNTSC). The authors would like to thank all of the individuals who contributed to this study. Among these were John Harding, Chief Scientist of the Maglev Technology Development Staff in the Federal Railroad Administration, and James Milner of Mitre Corp., who served as Technical Monitor for this project. At the Volpe Center, Robert Dorer, Chief of the High-Speed Ground Transportation Special Projects Office and Michael Coltman, Project Manager, supervised execution of the work.

Our major collaborators in this undertaking were staff members of Gumman Aerospace (now Northrop Grumman) in Bethpage, New York. Paul Shaw was Project Manager and Phil Danley was chiefly responsible for the development of the simulation software and computer-generated graphics. Dick Gran supervised the entire undertaking. Pilots Bill Patterson and Jim Dowd helped develop the experimental procedures and flew the pilot tests. John Eng, Dennis Brooks and Mike Yamond ran the simulator.

The New York State Energy Research and Development Authority provided some of the funding for the conversion of Gumman's X-29 simulator to Maglev use and for a study of the effects of vibration on passenger comfort. Richard Drake, its Program Manager for Transportation led that effort. Berger, Lehman Associates, under contract to Will Ristau of the New York State Thruway Authority, developed the alignment, which was the basis for the flight paths used in the experiments described in this report. Donald Baker and the New York State Department of Transportation provided additional technical assistance in this effort.

United Beechcraft Inc. of Farmingdale, New York provided the 1900C aircraft and crew used in the final series of flights. Bill Dolan made the arrangements, Ray Marciano supervised the equipment installation and flew the check flight, while Wayne Demming and John Bonuch, Jr. flew the nine experimental flights.

Carol Preusser and Bill Nissen of Preusser Research Group did their usual fine job of recruiting and managing subjects.

M. J. Griffin supplied expert advice and consultation on development of the ride-quality model, based upon his seminal work in the field.

Finally, we would like to thank our experimental subjects, whose responses form the basis of this entire report. We are especially grateful to those who experienced some symptoms of motion-sickness, but stuck with the experiment to the end. 


\section{Table of Contents}

1. Executive Summary 1

2. Introduction 5

2.1 Background and Objective 5

2.2 Motion Sickness Dose Value $\quad 8$

3. Approach 10

3.1 Modeling the Hypothetical Route 10

3.2 Selection of Test Vehicles $\quad 12$

3.3 The Flight Experiment 13

3.4 The Simulator Experiment 15

$\begin{array}{lc}3.5 \text { Subject Selection } & 18\end{array}$

3.6 Experimental Procedure $\quad 18$

3.7 Aircraft Instrumentation $\quad 26$

3.8 Simulator Instrumentation $\quad 29$

4. Analysis of Data $\quad 30$

4.1 Description of motion-sickness dosage value $\quad 30$

4.2 Analysis of Flight Data 33

4.3 Discussion of lack of correlation between MSDV \& subject ratings 37

4.4 Discussion of Correlation between Duration and Subject Ratings 44

4.5 Comparison of Flights with Simulations $\quad 45$

$\begin{array}{ll}\text { 5. Conclusions } & 49\end{array}$

Appendix A. COMBO.BAS: Overview and Explanation 53

Purpose $\quad 53$

Overview $\quad 53$

Description of Input $\quad 53$

Description of Output $\quad 54$

Introduction to Program Architecture 54

Description of COMBO.BAS: Program Flow Control and Algorithms 55

$\begin{array}{ll}\text { Appendix B. COMBO.BAS Annotated Code } & 60\end{array}$

Appendix C. KINCALC.SAS: Brief Description and Program 78

Appendix D: ALIGNMENT.BAS and New York State Data 83

Appendix E: Plot of bank angle and roll rate vs. time for the worst case $\left(28^{\circ}\right.$ and $\left.8^{\circ} / \mathrm{sec}\right) \quad 88$

Appendix F. Subject Consent Form 91 


\section{List of Figures}

Figure 3-1. Exterior view of the test aircraft.

Figure 3-2. Interior view of the test aircraft.

Figure 3-3. Interior view of the Northrop-Gumman Maglev simulator.

Figure 3-4. Exterior view of the Northrop-Grumman Maglev simulator.

Figure 3-5. Examples of first two pages of subject rating booklet.

Figure 3-6. Partial aeronautical chart showing the warning area where tests were conducted.

Figure 3-7. Attitude display temporarily installed in the cockpit of the Beechcraft 1900C.

Figure 3-8. Notebook computers used for data acquisition and generation of the cockpit display.

Figure 3-9. Example of the correspondence between the desired bank angles and the actual bank angles during a three-minute period. Desired bank angle (DBA) is shown in black, while actual bank angle (CBA) appears in gray. The deviations in the actual are primarily the results of turbulence and pilot actions in this example.

Figure 4-1. Motion-sickness dosage for 27 hypothetical combinations of bank-angle and roll-rate limits for the New York State Thruway route.

Figure 4-2. Passenger ratings of ride comfort during the flight experiments as "heutral" or worse (4 or greater on the 7-point scale).

Figure 4-3. Passenger ratings of ride comfort during the flight experiments as "Somewhat comfortable" or worse ( 3 or greater on the 7-point scale).

Figure 4-4. Passengers reporting "Slight queasiness" or worse (3 or greater on the 7-point scale). 35

Figure 4-5. Roll rates and accelerations for Flight 8.

Figure 4-6. Spectrum of the vertical acceleration record shown in Figure 4-5. The vertical scale is expressed in decibels.

Figure 4-7. Passenger ratings of ride comfort summed across all flights(1=very comfortable, 7=very uncomfortable).

Figure 4-8. Passenger ratings of motion sickness summed across all flights. 39

Figure 4-9. Motion sickness ratings by cumulative dose. 43

Figure 4-10. Scatter plot of motion sickness ratings for individual intervals. . 44

Figure 4-11. Motion sickness ratings by interval, summed across flights. 45

Figure 4-12. Comparison of motion sickness ratings between the airplane and the simulator. $\quad 46$

Figure 4-13. Comparison of ride-comfort ratings for flights vs. simulator trips. 47

Figure 4-14. Roll rates and accelerations experienced in the simulator for the worst case. $\quad 48$ 


\section{List of Tables}

Table 3-1. Combinations of limits on bank angles and roll rates.

Table 4-1. Characteristics of the nine test trajectories: Bank angle, roll rates, MSDVz (actual and desired), average speed and transit time for $277 \mathrm{~km}$ (172 miles) of the New York State Thruway.

Table 4-2. Comparison of two linear models fitted to the motion sickness ratings.

Table 4-3. Dosages and subject responses for the nine flights.

Table 4-4. Correlation matrix for dosages and subject responses. 


\section{Executive Summary}

Proposed high-speed ground transportation systems, such as Maglev, may have motion characteristics affecting passenger comfort which set them apart from anything previously experienced. Operating at aircraft speeds along rights-of-way established for conventional ground vehicles, Maglevs may subject passengers to significantly larger vertical accelerations and roll rates than they have ever felt on existing common-carrier modes. If the design limits for guideway curvature are set too high in the interest of achieving the shortest travel times and/or maximum utilization of existing, short-radius right-of-way, substantial numbers of passengers may find the ride quality unacceptable because of excessive vertical acceleration and roll rates. In that case, speed would be reduced, resulting in moderately longer trip times. In areas where new right-of-way is unavailable, the question becomes how can a Maglev guideway be optimally fitted to it and what speeds should be used.

Previous research carried out by the Volpe Center for the National Maglev Initiative demonstrated that more than $95 \%$ of the public would accept isolated Maglev maneuvers involving bank angles up to $37^{\circ}$ and roll rates up to $7 \%$ sec. Since these limits were higher than those contemplated in most Maglevsystem-design proposals, passenger acceptance did not appear to impose any significant constraints. However, further reflection on motion sickness as experienced in other modes suggests that the frequency of occurrence of motions, as well as their power spectra, are as important as their magnitude and that what passengers see may strongly influence their likelihood of becoming ill. Hence this study was undertaken to explore comfort and motion-sickness effects of Maglev travel in corridors characterized by frequent curves.

Four segments of the New York State Thruway, totaling $277 \mathrm{~km}$ (172 miles), were chosen as the hypothetical route for evaluating passenger acceptance for the following reasons:

- these segments are representative of a great deal of the hilly terrain found in the United States, - their length of $277 \mathrm{~km}$ ( 172 miles) is typical of the distance between several major city pairs which would be good candidates for Maglev service, - the State of New York was willing to supply detailed maps containing the required data to construct the hypothetical route, and

- the State of New York provided significant financial support to the construction of the simulator used for part of this study.

Route alignment data from the aerial photos and engineering drawings were coded and published by Berger, Lehman Associates. These were input to a set of computer models which generated files containing the exact bank angles at intervals of 0.1 second of a hypothetical Maglev following the Thruway. Alternative files were generated for various assumptions about maximum allowable bank angle, maximum allowable roll rate and the longitudinal acceleration and deceleration characteristics of 
the vehicle. These various sets of assumptions implied travel times over the $277 \mathrm{~km}$ (172 mile) route of 39 to 49 minutes. Bank angles as high as $40^{\circ}$ and roll rates as high as $12 \% \mathrm{sec}$ were considered.

To facilitate both the experimental design process and subsequent data analysis, a procedure was developed for estimating the propensity of a given set of ride motions to induce motion sickness. This procedure is based upon the work of M. J. Griffin and British Standard 6841:1987 for ride quality. It generates a number called the Motion Sickness Dosage Value (MSDV), from which the proportion of passengers who will experience nausea can be estimated. The model predicts the incidence of kinetosis from the magnitude and duration of exposure to low-frequency $(0.1-0.5 \mathrm{~Hz})$ vertical accelerations. For the hypothetical route, 27 alternative sets of design limits for bank angle, roll rate and longitudinal acceleration and deceleration were initially considered, which had MSDV scores ranging from less than 2 to 13 . British Standard 6841 provides an approximate method for convenient interpretation of these figures. In a "mixed population of unadapted male and female adults"BS 6841 gives the estimate:

$$
\text { Percentage of persons who may vomit }=1 / 3 * \text { MSDV. }
$$

Also, the scores may be used for comparative purposes; motions leading to high MSDV scores may be expected to produce more motion sickness than motions leading to low scores.

The only means of simulating trips with realistic accelerations at reasonable cost is through the use of an airplane. In turning, aircraft naturally bank at just the right angle to eliminate.lateral forces on the passenger, just as a Maglev would. Conventional ground vehicles would produce unpleasant and unrealistic lateral accelerations in rounding turns at high speeds, since they are restricted to low amounts of super-elevation and generally lack tilt-body suspensions.. The principal disadvantage of using an airplane as a simulator is that it cannot provide a realistic out-the-window view a future Maglev passenger would see. Only a laboratory simulator can safely expose passengers to the visual effects of scenery rushing by at 400 kilometers per hour (about 250 miles per hour) at ground level. The laboratory simulator can also add realistic amounts of vibration.

To provide facilities for testing subjects in both the airliner and laboratory simulations, a contract was awarded to Grumman Aerospace Inc. (now Northrop Grumman Corp.). This contract supported the development of computer-generated-imagery of the New York State Thruway right-of-way, use of the simulator and staff for testing subjects, and use of a 21-seat Gulfstream I and crew for flight experiments. Due to the merger with Northrop and the ensuing downsizing of the corporate fleet, this aircraft was replaced by a Beechcraft $1900 \mathrm{C}$.

An experimental apparatus was constructed to facilitate flying an airliner through a series of several dozen roll maneuvers which would subject passengers to the same vertical accelerations and roll rates they would experience in a Maglev built to a given set of design standards. This apparatus was based upon two notebook computers linked to a roll-rate gyro and a three-axes accelerometer. It generated a cockpit display showing what the aircraft's bank angle was supposed to be at any given time, what its actual bank angle was, and the direction of the next maneuver. The pilot's job was simply to keep the two bars on the display parallel. The apparatus also recorded the outputs of the accelerometers and 
rate gyro at 0.1 second intervals, thus allowing MSDV and other measures of ride quality to be calculated.

After training the crew to fly the experimental procedures and securing use of restricted airspace, two preliminary tests were conducted using government and contractor personnel as subjects. These tests exposed subjects to two intervals of flying with relatively high bank angle limits, consistent with making the $277 \mathrm{~km}$ ( 172 mile) trip in about 38 minutes. More than half the subjects began feeling queasy at these higher limits. As a result, a decision was reached to restrict the exposure of subjects drawn from the general public to bank-angles of less than $30^{\circ}$ and roll rates of less than $9 \%$ sec.

The final experimental design specified nine flights with 14 subjects each. Each flight simulated a 277 $\mathrm{km}$ trip made with one of the nine possible combinations of limits for bank angle and roll rate. The limits for bank angle were 14, 21 and 28 degrees while those for roll rate were 4,6 and 8 degrees per second. Since the laboratory simulator seated only four subjects, two sessions were conducted with each combination of limits, allowing more than half of the persons who had flown to take the simulator trip as well. Subjects were required to rate ride comfort and their own tendency to motion sickness (both on seven-point scales) five times during both trips and to read magazine articles and answer questions about them.

Analysis of the data from the subject rating sheets and the instrumentation lead to the following conclusions:

1. Cumulative dosage and duration of exposure showed significant correlation with motionsickness ratings. The implication of this finding is that average values for bank-angle and rollrates should be lower on longer routes than on short ones.

2. Within the bank-angle and roll-rates limits tested, the vast majority found the plane ride comfortable and felt no motion-sickness. These limits were consistent with those required of the Maglev Systems Concepts Developers.

However, a significant minority, $8 \%$, felt intermittently nauseous or worse during some portion of the flight and two subjects vomited. $23 \%$ felt slightly queasy at some time during the flight. Differences between subjects in their perceptions of ride quality and propensity for motion sickness appear to have swamped the physical effects of the differences in bank-angle and rollrate limits for different flights. Ratings of ride comfort and motion sickness were not significantly correlated with bank-angle or roll-rate limits.

The percentages of passengers showing signs of motion sickness in the flight experiments are probably greater than the percentages who would do so aboard an actual Maglev, because the flights subjected them to somewhat larger doses of vertical acceleration than they would have received aboard a Maglev with the same nominal bank and roll limits. Furthermore, the limited 
views through the small airplane windows and/or anxiety about the flight may have contributed to the onset of nausea in some subjects. Hence the foregoing conclusions are conservative.

3. The MSDV Model predicted that 1.92 subjects would vomit, given the accelerometer data from their flights. That two subjects actually vomited suggests that the model is useful in evaluating route alignments.

4. In the laboratory simulation, no subjects vomited and only one of 71 reported even intermittent nausea. Thus the visual effects of scenery rushing by at $400 \mathrm{kph}$ ( $250 \mathrm{mph})$ do not appear to present a problem when that view is limited to a side window, even as large as the 89 $\mathrm{cm}\left(35^{\prime}\right)$ video monitors used in the experiment. 


\section{Introduction}

\subsection{Background and Objective}

The development and evaluation of proposed Maglev transportation systems have been predicated upon the use of existing rights-of-way for some of the system route mileage. This constraint was expressed by Congress in the Intermodal Surface Transportation Efficiency Act Of 1991 (ISTEA), which states:

It is the policy of the United States to establish in the shortest time practical a United States designed and constructed magnetic levitation transportation technology capable of operating along Federal-aid highway rights of way, as part of the national transportation system of the United States.

The assumption of use of existing right-of-way is also a reflection of the fact that in the corridors between large cities, which are the primary candidates for Maglev routes, land values may be so high as to make it impractical to acquire large amounts of new right-of-way.

Because the existing rights-of-way were laid out for speeds below $160 \mathrm{kph}$ (about $100 \mathrm{mph}$ ), the radii of curves and the lengths of spirals (segments of guideway where radii are changing from infinity to those of the curved segments) are sub-optimal for Maglevs or other very high-speed, fixed-guideway systems, operating at more than twice the maximum speed of existing ground systems. To negotiate curves at Maglev speeds, the vehicles must bank as aircraft do for reasons of both passenger comfor and to minimize lateral forces on the suspension and guideway structure. The centrifugal force developed in these curves and spirals will be resolved and experienced by passengers as positive vertical acceleration ( $\mathrm{g}$ loading) just as in airplanes. For a curve of given radius, the faster the design speed for a Maglev guideway, the greater the bank angle must be and the greater the extra vertical g-force acting on the vehicle and passengers. For a spiral of given length, the greater the Maglev's speed, the greater the roll rate it will experience in traversing the spiral. Roll rate can be perceived as the rate of change in vertical acceleration. Since centrifugal force increases as the square of velocity, it becomes apparent that while it may be hardly noticed on the curves of Interstate Highways at normal passenger-car speeds, at $400-500$ $\mathrm{kph}(250-300 \mathrm{mph})$ it can amount to several tenths of a $\mathrm{g}$.

Recognition of these implications of guideway alignment leads to the following questions:

- What are the comfort limits for acceleration (lateral, vertical and longitudinal)?

- What are the comfort limits for roll rates and bank angles? 
- What are the effects on comfort of sustained exposure to various accelerations and roll maneuvers (as opposed to situations in which such forces and maneuvers are encountered only in brief, isolated segments of a trip)?

- Does the visual environment which would be experienced by Maglev passengers introduce any additional concerns?

All of these questions are related to system design and economics in terms of right-of-way alignment constraints, forces acting on various components of the vehicles and guideways, average speeds attained, and a host of other issues.

Humans differ greatly in their perceptions of what constitutes a "comfortable" ride. Various aspects of ride quality, e.g., vertical acceleration and roll rates, seem to act synergistically in degrading perceived ride comfort. Existing tests and standards for ride quality were developed for other modes and translate poorly or not at all into a $500 \mathrm{kph}$ ground environment.

In attempting to answer these questions, the staffs of the National Maglev Initiative and the Volpe Center chose to begin with simplest ones:

- What are the tolerance limits of the public for individual, isolated maneuvers which generate positive or negative vertical acceleration alone?

- What are the tolerance limits for separated, coordinated tuming maneuvers, which generate both positive vertical acceleration and a rolling sensation, in terms of maximum bank angle and maximum roll rate?

These questions were addressed in the Study to Establish Ride Comfort Criteria for High Speed Magnetically Levitated Transportation Systems (Ref. 1). That study concluded that fewer than $5 \%$ of the public would hesitate to ride on a system in which maximum bank angles were limited to $37^{\circ}$ and roll rates were limited to $7 \% \mathrm{sec}$. Since these values were higher than those specified in most of the concepts then being developed, it seemed that on the basis of the experiments described in the report, ride-quality considerations might not constrain system design significantly.

However, the fact that many people experience kinetosis (motion sickness) under a variety of conditions on vehicles which are not violating the aforementioned limits, suggests the necessity of looking beyond comfort ratings for isolated maneuvers. ("Isolated" means that maneuvers were separated in time by at least one minute, with an average period between moments of peak acceleration of nearly two minutes.)

Furthermore, as many have learned through personal experience in recent years, simulators and virtualreality devices can produce symptoms of motion sickness in some individuals, even when there is little or no actual motion occurring. The authors of this report and staff from the National Maglev Initiative 
were provided with an opportunity to get a pilot's eye view of the world from an F-14 fixed-base simulator. At a simulated speed of $500 \mathrm{kph}$ and an altitude of about $20 \mathrm{~m}$ while observing combat maneuvers, most of us began to feel a bit queasy in just a few minutes while viewing the giant, $180^{\circ}$ field-of-view screen. Although we found we could mitigate nausea by restricting our fields of view to small portions of the total projected image, we recognized the need to conduct tests to quantify the effects of cumulative exposure.

The literature (Ref. $2,3,4$ ) shows that the development of motion sickness depends not only on the magnitude of the accelerations experienced but also on their frequency characteristics and duration. Hence, for a given speed, it is the angle of tilt of the guideway (plus any additional tilt developed in the vehicle's suspension system) which determines the magnitude of the vertical acceleration. The length of the spiral determines the roll rate and hence the spectral distribution of the acceleration. Accelerations with periods in the range of 0.06 to $0.5 \mathrm{~Hz}$ are the primary contributors to motion sickness. Accelerations with shorter periods are sensed as vibration. They may be uncomfortable, but seldom induce motion sickness.

There are certain important insights to be gained from the literature which have served to guide the design of this study:

1. Motion sickness develops when there is some incongruity among sensory inputs from the visual, vestibular and kinesthetic systems. One may experience frequent accelerations and rolling movements of the head in many sports, for example, without any fear of sickness. Yet if a subject were sitting in a motion simulator and were exposed to the same accelerations, he might quickly become ill. Conversely, the phenomenon of "simulator sickness" has been widely reported (Ref. 5, page 282-283). Subjects in simulators who are feeling little or no actual motion, but are exposed to a visual field which suggests rapid movement, frequently develop one or more symptoms of motion sickness.

2. Controlling one's vehicle is a powerful preventative for motion sickness. Thus drivers virtually never become car sick, while passengers may. The best cure for sea sickness is taking the helm. If an individual is not actively controlling a vehicle, looking out the window, especially at the horizon, helps ward off illness because it helps establish congruity between the various sensory inputs. Unfortunately, when passengers direct their visual focus toward reading, writing, operation of computers, etc., they effectively enhance whatever tendency they may have to motion sickness. Hence, common carriers catering to business travelers must provide smoother rides than user-operated modes.

3. Vertical motions with frequencies in the range from $0.06 \mathrm{~Hz}$ to $0.5 \mathrm{~Hz}$ are the primary ones of significance for motion sickness. More rapid motions (sensed as vibration) may cause discomfort and annoyance, but do not bring on nausea. Vertical accelerations induce more motion sickness than lateral or longitudinal accelerations of the same magnitude. 
4. The longer passengers are exposed to motions with characteristics which induce motion sickness, the higher the proportion of them that will develop symptoms. For motions that might realistically be encountered in Maglev systems, symptoms could begin to develop in the most sensitive individuals in less than 15 minutes, while others would remain symptom-free for hours longer than the transit time for any foreseeable Maglev corridor. For a constant motion characteristic, proportion of subjects experiencing vomiting is approximately proportional to the square root of the travel time up to about two hours.

\subsection{Motion Sickness Dose Value}

Motion sickness dose value (MSDVz) refers to a methodology for quantifying the motion sickness potential of a sequence of vertical accelerations. This internationally accepted measure is described in the new ISO 2631 (Annex C) on Mechanical vibration and shock - Evaluation of human exposure to whole-body vibration (Ref. 6). The method involves computing a weighted root-mean-square (vertical) acceleration. The weighting is designed to attenuate accelerations that are not in the frequency range from 0.06 to $0.5 \mathrm{~Hz}$. MSDVz was derived from British Standard 6841 .

A body of literature exists supporting the use of MSDVz as a measure of motion sickness potential relevant discussion can be found in Handbook of Human Vibration by M.J. Griffin (Ref. 5). Several studies have investigated MSDVz on ships. While motion sickness often occurs in planes, cars and other vehicles, the low-frequency vertical accelerations captured by MSDVz have been most common only in the marine environment. Thus the measure is of limited use in quantifying motion-sickness potential of aircraft and even less utility with respect to conventional ground vehicles. The causes of motion sickness are varied, and MSDVz is designed to assess a particular, known cause. In fact, ISO 2631 warns "The methods ... should be primarily applicable to motion in ships and other sea vessels".

Unlike traditional steel-wheel, steel-rail passenger systems which generate relatively low levels of vertical acceleration, modern high-speed, fixed-guideway systems could potentially produce substantial low-frequency vertical acceleration while traversing a sequence of curves. Through tilt technology, banked guideways or a combination thereof, the accelerations experienced by a passenger may be resolved through the vertical axis. A question of interest is whether the MSDVz, which takes as input data only the magnitude and duration of accelerations in the $0.1-0.5 \mathrm{~Hz}$ range, would be an appropriate tool for such a system.

The present study makes use of the MSDVz measurement technique in two ways. First, to aid in designing the study, MSDVz was estimated for each condition. Second, the MSDVz is used in data analysis: MSDVz was used as a predictor of the subjects' ratings. Furthermore, details of the calculation of the MSDVz, based on a measured sequence of accelerations and also based on a hypothetical route, are given in the Appendices. 
It is important to realize that ISO 2631 provides no absolute guidance regarding the MSDVz measure, only relative guidance. Only with regards to the percentage of people who would vomit is there any absolute basis for evaluating the measure. The ISO reports that "...for a mixed population of unadapted male and female adults" the percentage of people "who may vomit" is $1 / 3 \mathrm{MSDVz}$. This prediction was investigated in the current study. 


\section{Approach}

Because of the large number and complexity of the variables which influence the development of motion sickness, and because of the large differences among individuals in terms of susceptibility to that illness, an experimental design which attempted full-factorial treatment of variables would be impossibly expensive. Very early in course of this study, a decision was reached to test subject responses to sets of motions which resemble as closely as possible those of hypothetical Maglev vehicles operating over actual terrain. All tests would simulate passage through the same terrain, but the limits for maximum bank angle and roll rate would be varied. As higher limits for these variables are allowed, higher average speeds through turns are achieved. Thus the results of the test could be expressed essentially as a tradeoff between travel time over an actual route and passenger comfort.

\subsection{Modeling the Hypothetical Route}

The ride quality alignment model was developed to provide an aircraft pilot with a sequence of maneuvers which will simulate during flight, ride quality typical of a Maglev vehicle operating over a realistic guideway. In designing the model and selecting the route, the following criteria had to be met:

1) a realistic Maglev guideway alignment of more than $160 \mathrm{~km}$ (100 miles) in length including detailed descriptions of guideway vertical and horizontal curvature at a scale of $l^{\prime \prime}=500$ or finer;

2) including multiple terrain types;

3) following an existing right-of-way; and

4) output from the model in a form which could be readily converted into a cockpit display.

The State of New York commissioned a study (Ref. 7), in which a Maglev guideway geometry was developed along four sections of the New York Thruway and fit within the existing right of way. The four sections reported as appendices in the report are:

$1 \& 2$ ) The Thruway main line (1-87/90) (Appendix $F$ begins between interchange \#16 and \#17 and ends between interchange $\# 20$ and $\# 21$ ) (Appendix $G$ begins between interchange $\# 30$ and $\# 31$ and ends just beyond interchange \#34A ),

3) I90 from Manchester to Rochester (Appendix $H$ begins at about interchange $\# 42$ and ends at just before interchange \#47) and 
4) The Berkshire Section (I-90) to the Massachusetts State Line (Appendix I begins at about interchange \#B1 and ends just before interchange \#B3).

These four sections represent multiple demographic, topographic and terrain types. The guideway geometry was available as engineering drawings $\left(1^{\prime \prime}=500^{\prime}\right)$ and as data in the final report. However, neither the drawing, nor the New York report specified individual spiral lengths or spiral start/end locations.

The Ride Quality Alignment Model reconstructed two dimensional spirals based on a combination of information supplied by the New York Thruway Authority, Berger Lehman Associates, the design drawing and the report. Output from the model was in a form which could readily be displayed in the cockpit.

The Ride Quality Alignment Model has a coherent, fully extensible modular architecture. The six functional sections are: Alignment, Balance, Acceleration, Deceleration, Roll Rate, Bank Angle (as a function of arc length), Travel time (reparameterize in time) with smoothing. Modular subsections are arranged to automatically report constraint(s) which modify speed.

It was assumed that the study aircraft would fly at constant speed using smoothly transitioning maneuvers. No explicit vehicle characteristics (aerodynamic or propulsion technology) were considered. Acceleration and deceleration values were assumed equal. It was assumed that the radii of curvature reported in the New York Study and input to the model described the radius at the apex of each curve. The four sections of guideway developed by the New York State Study were input as one continuous set.

The modeling approach was to first "build" the guideway and then "move" backward and forward over the entire track while computing the speed which satisfies physical laws and human-factors constraints. Backward and forward movement over the route ensures that interactions among sequences of curves will be fully modeled.

Locally the program "looks ahead" to the next piece of track, to ensure smooth speed transitions from one small piece of track to the next. The resulting speed profile was assumed smooth, and jerk was not explicitly modeled.

A speed profile for the entire route was computed separately for unconstrained or balanced lateral forces, for constrained lateral forces, for the induced vertical forces, and for longitudinal forces. As each force was computed the value of speed which satisfies that constraints was compared to the previous lowest computed speed value at that point in space. If the new speed value was more constraining i.e., lower, speed was adjusted. The final speed profile satisfied all the considered constraints. Each time the speed profile was adjusted, the constraint which led to reduced speed was noted. 
By taking advantage of symmetry, the Acceleration and Deceleration Modules and Reverse 1 and Reverse 2 Modules contained duplicate code. By choosing to implement code as a function of space rather than the traditional parameterization in time, the code was simplified. Outputs were the values of: (1) speed at every point on the horizontal guideway as a function of space, (2) speed at every point in time and (3) the most influential speed constraint at every point. After these values were calculated for the hypothetical Maglev trip profiles, they were used as inputs to a program which computed desired bank angles and roll rates for an aircraft flying at constant speed, which would subject passengers to the same amounts of vertical acceleration and roll at each moment of the trip.

The appendices contain a more detailed description of the model and a code listing.

\subsection{Selection of Test Vehicles}

The only available test vehicle which can come close to simulating the ride characteristics of a Maglev is an airplane flying through smooth air. As noted in the previous section, traversal of a curving rightof-way like the New York State Thruway at speeds of around $400 \mathrm{kph}(250 \mathrm{mph})$ generates centrifugal forces ranging up to about $0.2 \mathrm{~g}$. To avoid unpleasant lateral forces on passengers and excessive lateral loads on suspension, a Maglev can be designed so that it always banks at an angle which produces a coordinated turn, i.e., one in which the lateral force seems to disappear. Airplanes do this naturally; hence objects remain on tray tables, drinks do not spill, and passengers perceive no side forces as airliners bank and turn.

No practical ground-based simulator can reproduce the accelerations acting on passengers in a Maglev, because nearly all of them are positive. Thus the simulator would need to be miles high in order to generate an hour-long sequence of realistic, positive vertical accelerations. A wheeled vehicle following an appropriate, steeply banked course at the correct speed could generate the required vertical accelerations, but the guideway would be expensive to build and several versions would be required in order to test various speed profiles. Existing test tracks and race tracks would not produce the required pattern of $g$-forces, nor could a bus-like vehicle be driven fast enough to generate them.

The visual effects of seeing scenery at ground level rushing by at $500 \mathrm{kph}$ were regarded as a potential problem of significant proportion for Maglev passengers. Since there was no feasible and safe method to test for visual effects at the same time subjects were experiencing realistic accelerations in an aircraft, a separate series of experiments in a ground-based simulator was devised. These would provide roll motions, visual effects and even simulated longitudinal accelerations based on the characteristics of the New York State Thruway route at various limits for roll rate and bank angle. Their prime objective was to determine whether the out-the-window view would induce kinetosis in any subjects. 


\subsection{The Flight Experiment}

The principal disadvantages of the airplane as a Maglev simulator are that it can introduce unwanted motions (e.g., turbulence effects), that it can not be safely flown at an altitude that produces a realistic out-the-window view, that it is noisy, that some persons find it inherently frightening, and that it cannot provide longitudinal acceleration or mid-frequency vibrations. The first of these can be minimized by flying only in calm air, generally above 3657 meters (12,000 feet) when no storms are present. There is no practical solution to the second problem, other than a separate experiment (described below in Section 3.4). The third can be mitigated by using a turbine-powered airliner, as opposed to smaller, piston-powered craft.

Since a contract was being negotiated with Grumman Aerospace for the use of its Maglev simulator and staff for the experiments described below, it was efficient to include rental of a Grumman corporate aircraft, a Gulfstream I 21-seat aircraft for the flight experiments. The contract was written for this aircraft and initial crew training flights and pilot tests were conducted with this plane.

After the training one of Grumman's corporate air crews for about six hours (two hours in a simulator and four hours in flight), two preliminary test flights were conducted using employees of the U.S. Department of Transportation, State of New York, other Federal agencies and contractors as subjects. These took place on March 11 and April 12, 1994.

These tests consisted of a series of ten-minute intervals in which bank angle limits and roll rates were raised to progressively higher values. By the intervals in which the limits reached $30^{\circ}$ and $12 \% \mathrm{sec}$, most of the subjects felt queasy or worse. There was general agreement in the debriefing sessions that members of the general public should not be subjected to rides as unpleasant as those the preliminary test subjects had experienced. Lower limits, described below, were selected by the project team, based on these preliminary-test reactions.

Due to Grumman's merger with Northrop and subsequent corporate restructuring in the summer of 1994 , the Gulfstream I was sold. Gumman arranged to rent a Beechcraft $1900 \mathrm{C}$, pictured in Figure 31 , as a replacement. Figure 3-2 shows the interior of this aircraft. United Beechcraft, Inc. of Farmingdale, NY supplied the crew, who were trained in the course of two flights of about two-hours duration each in August, 1994. 
Figure 3-1. Exterior view of the test aircraft.

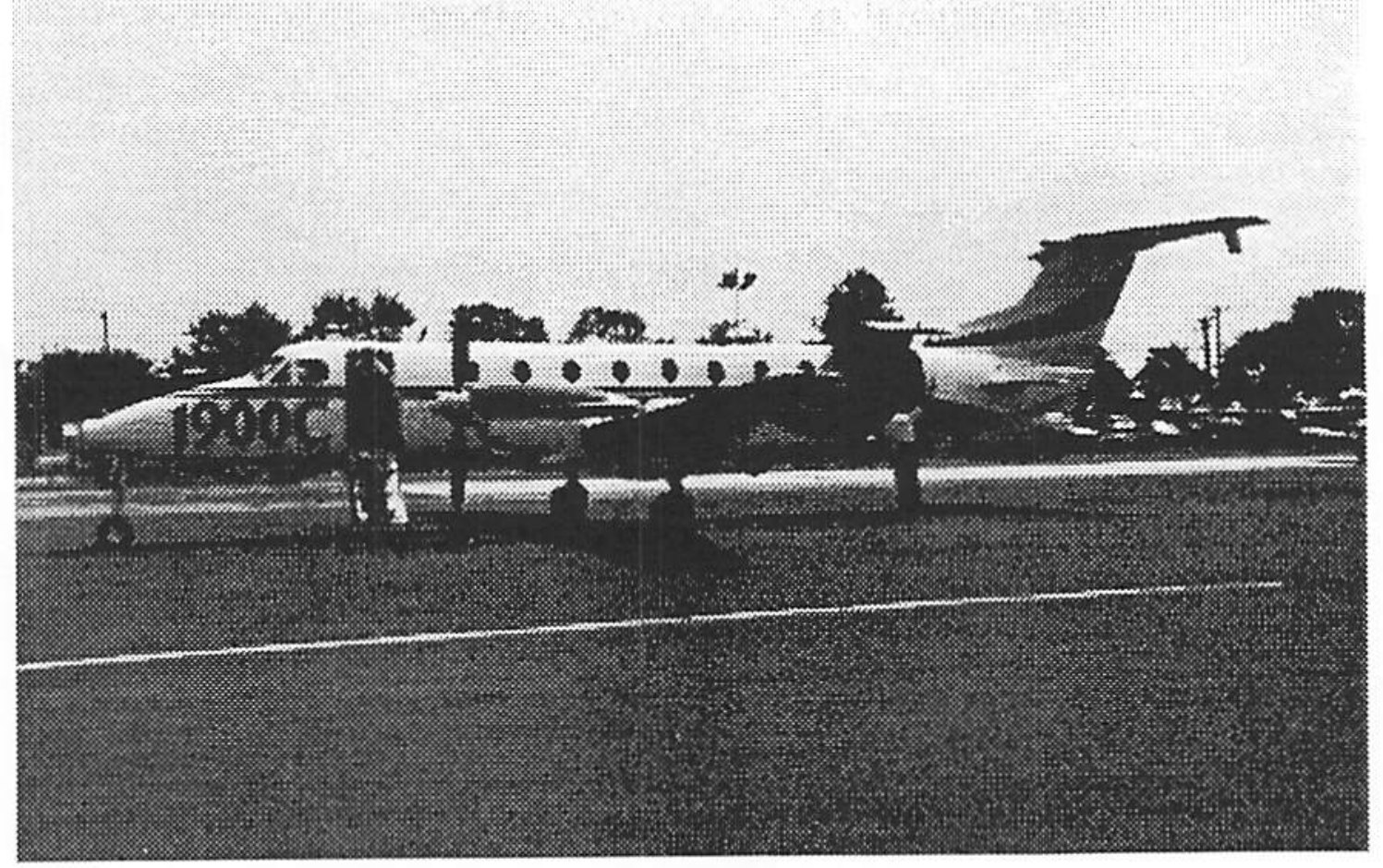

Figure 3-2. Interior view of the test aircraft.

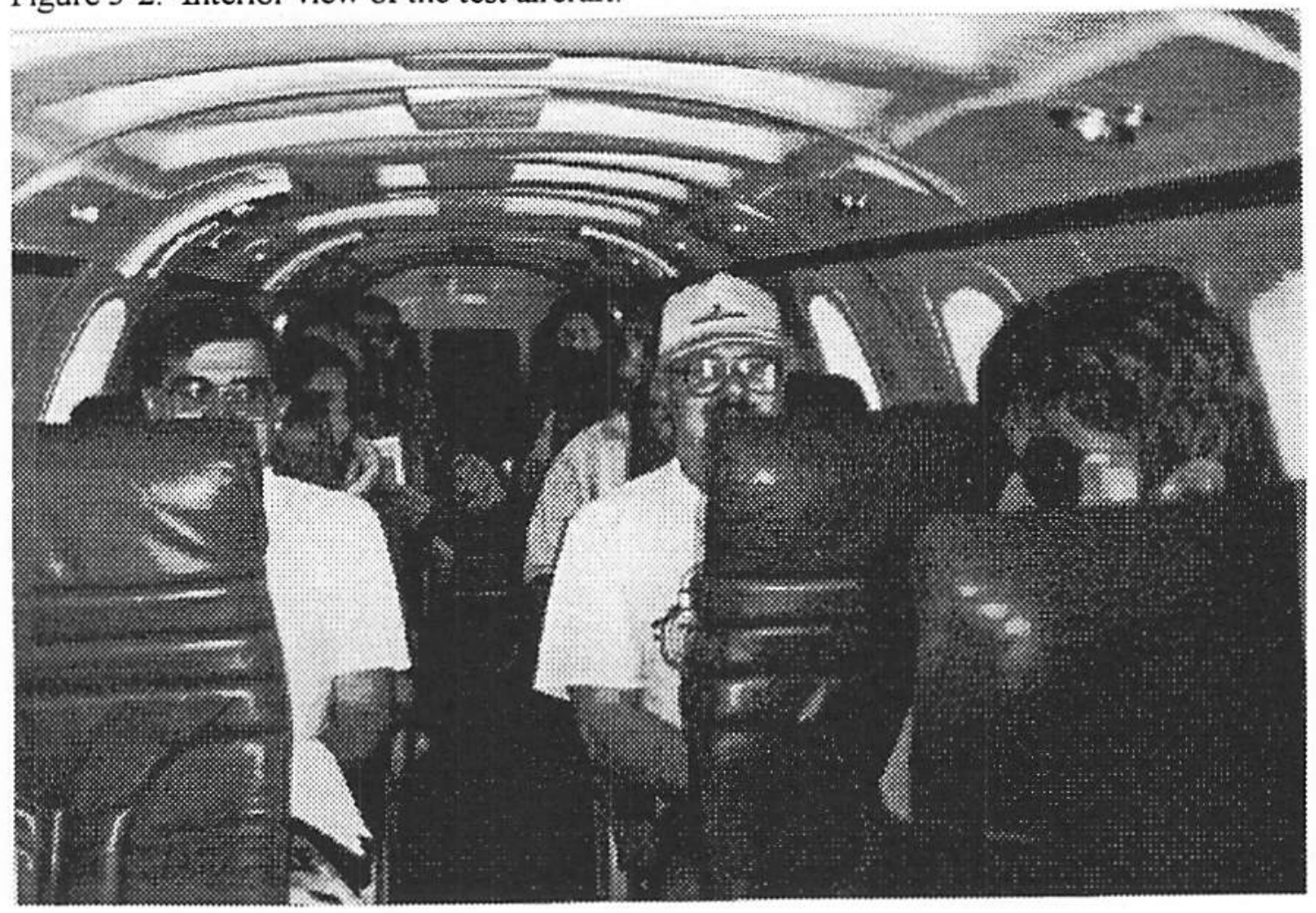




\subsection{The Simulator Experiment}

Following the termination of the X-29 experimental fighter program, Grumman Aerospace was left with a multi-million dollar, full-motion-base simulator with elaborate computer-graphics capabilities. When Grumman became active in Maglev development work, this simulator was converted to study passenger reactions to various aspects of the ride quality of Maglev or other transport vehicles.

This simulator contains a passenger compartment about 3.66 meters (12 feet) in overall length, which resembles a portion of the first-class cabin of an airliner with four seats. 35" video monitors are fitted at both windows to present computer-generated views coordinated with the simulated movements of the module. Figure 3-3 shows an interior view of the module.

The seats and vestibule are enclosed with a hemispherical dome with a radius of about 3.05 meters (10 feet). The entire assembly is mounted on a array of hydraulic cylinders, as shown in Figure 3-4. These cylinders are powered by a set of hydraulic pumps through control valves operated by computers in an adjacent room.

The simulator experiments were driven with the same computer files of bank-angle versus time data as were used in the aircraft experiment. However, since the simulator cannot produce sustained accelerations, the physical rolls were limited to about nine degrees in order to avoid subjecting the passengers to excessive lateral forces. Simulator motions mimicked the onset of a roll to higher angles and the out-the-window view in the monitors showed whatever angle of tilt was specified in the source file.

Because the creation of computer imagery is one of the significant cost elements in a simulation, only about $80 \mathrm{~km}$ (50 miles) of scenery were generated. These were repeated as necessary to provide a trip with a total length of $277 \mathrm{~km}$ (172 miles), just as the subjects experienced in the flight environment. 
Figure 3-3. Interior view of the Northrop-Grumman Maglev simulator.

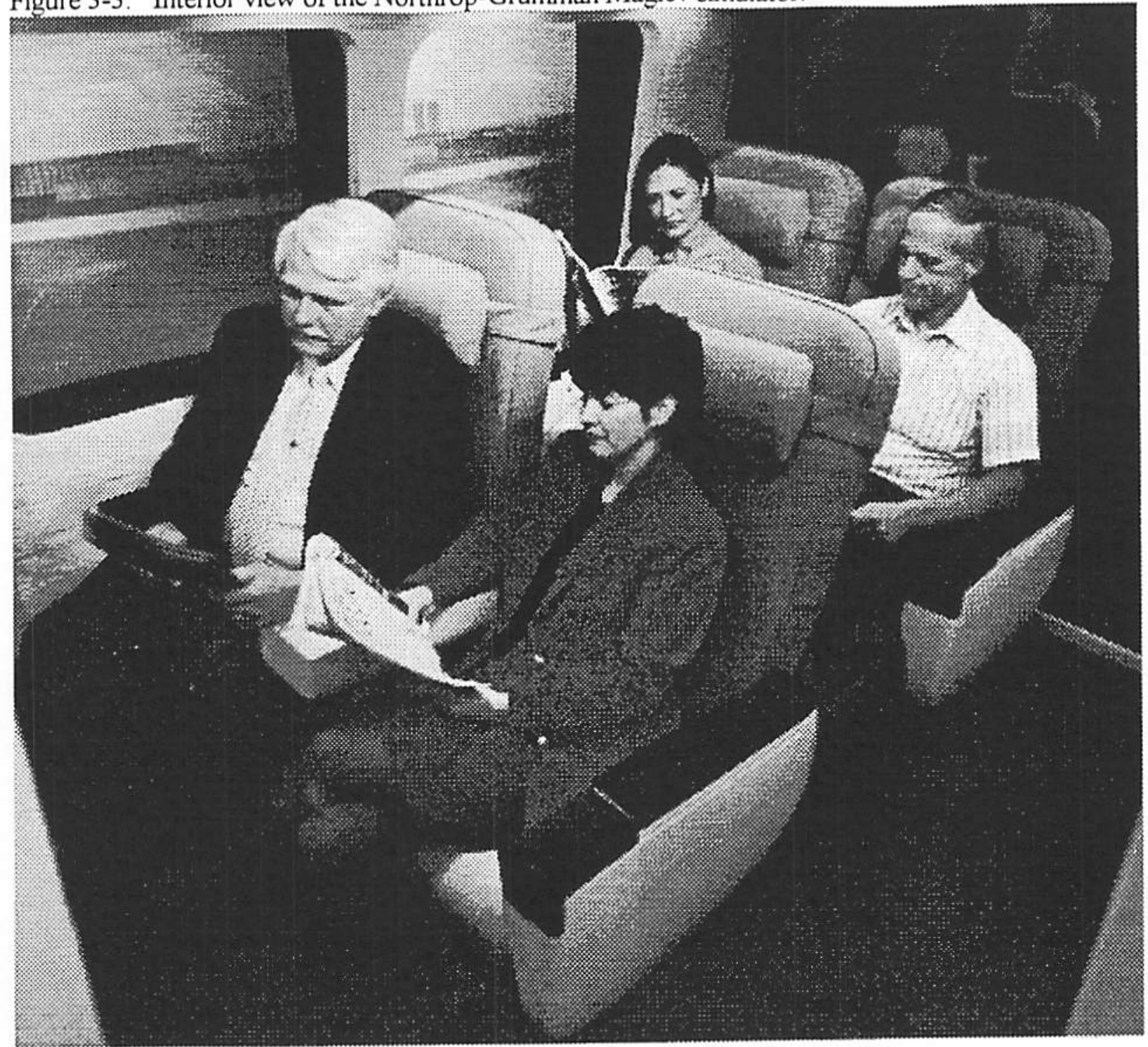


Figure 3-4. Exterior view of the Northrop-Grumman Maglev simulator.

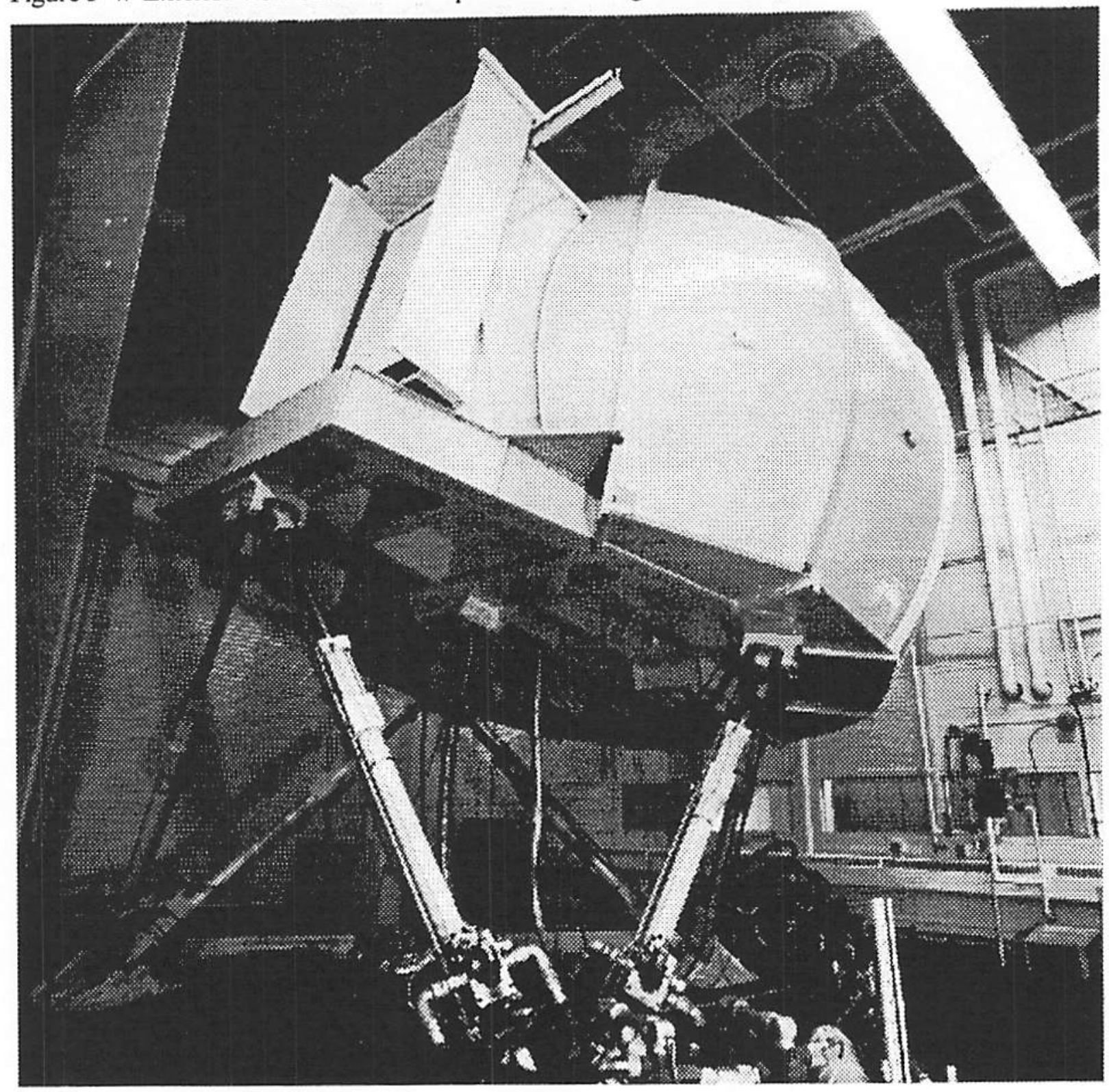




\subsection{Subject Selection}

Preusser Research Group, Inc. , was retained to recruit, screen, and select at least 16 subjects for each of the nine test flights. Fourteen were intended to make each flight, while two extras were recruited to compensate for no-shows.

The subject pool was roughly balanced with respect to age (18 to 65 years) and sex, but excluded persons who have not made at least six round trips by air, including at least two in the past year. Persons with any medical condition which might lead to injury due to flying or g-loading ( heart conditions, pregnancy, middle or inner-ear problems, etc.) were also excluded. Subjects selected were required to drive themselves to Republic Airport and were required to be somewhat flexible as to scheduling. Flights were subject to rescheduling for any of the following reasons: (1) bad weather or rough air in the test zone, (2) test area unavailable due to military use, (3) aircraft in use for other business, or (4) aircraft out of service for maintenance.

\subsection{Experimental Procedure}

In the lounges at the airport and at the simulator facility, contractor personnel briefed subjects and explained the way subjects were to evaluate each segment of the flight or simulator trip. Figure 3-5 shows the first two pages of the rating booklet the subjects were given. The following pages were similar except that the subject description items were deleted. It was explained to subjects that they would be expected to complete one rating sheet at the beginning of the experimental portion of the trip and one additional sheet each time they were prompted to do so by the experimenter. There were five such prompts on each flight, so that the rating intervals ranged from about eight to almost ten minutes in length. 
Figure 3-5. Examples of first two pages of subject rating booklet.

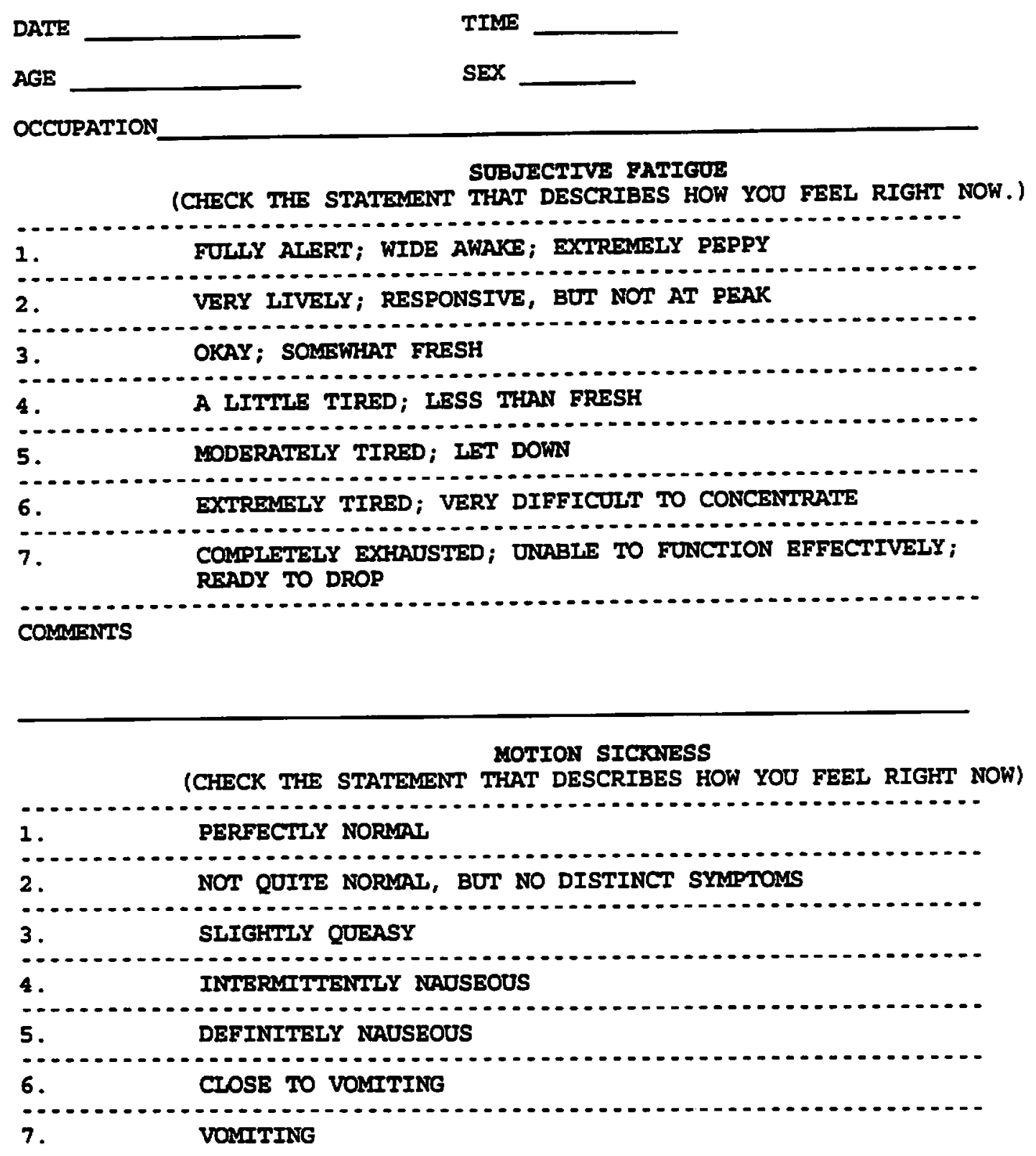


INTERVAL \#1

THE RIDE DURING THE PAST FTVE MINUTES WAS:

— VERY COMFORTABLE
COMFORTABLE
SOMIWHAT COMFORTABLE
NEUTRAI
SOMIWHAT UNCOMFORTABLE
UNCOMFORTABIE
VERY UNCOMFORTABLE
(PIEAASE CHECK ONLY ONE ANSWER)

NOTION SICTARESS

(CHECK THE STATEMENT THAT DESCRIBES HOW YOD FEEI RIGHT NOW)

1. PERFECTLY NORMAL

NOT QUITE NORMAL, BUT NO DISTINCT SMMPTOMS

-

$3 . \quad$ SLIGHTLY QUEASY

$4 . \quad$ INTERMITTENTLY NAOSEOUS

D.

6.

$6 . \quad$ CLOSE TO VOMITING

6.

7 VOAITING

Check here if you chose not to read because of queasiness.

What are a few of the names of the types of pianos mentioned in the article? 
At the lounges, they were also presented with a assortment of magazine articles to read during the experimental portion of the flight. Each package contained five articles of a few pages each, one for each of the five intervals. Subjects were required to read and answer in writing one question about each of the articles they read. Subjects were free to choose from the following categories of articles: (1) business, (2) entertainment, (3) fashion, (4) home and family, (5) science, (6) sports, and (7) miscellaneous.

Prior to leaving the lounges they were required to read and sign a consent form describing the experiment and its goals and risks. Copies of the forms are reproduced in Appendix F. Subjects were accompanied on each flight by two members of the research team and on each simulator trip by one Northrop Grumman staff member. Their schedule for one day was as follows:

\section{DAIIY SCHEDULE}

09:15

09:30

09:40

09:50

09:55

$10: 00$

10:05

$10: 30$

$10: 45$

10:55

11:00

11:05
8 morning simulator subjects arrive at security for check in.

Simulator subjects are escorted to simulator building.

Morning flight subjects arrive at the boarding lounge for briefing and use of rest rooms.

First group of simulator subjects are briefed. Second group remains in conference room to read or watch video.

Subjects enter simulator.

Simulator nun begins.

Flight subjects board aircraft.

Aircraft departs gate.

Aircraft takeoff.

Aircraft reaches 4572 meters $(15,000$ feet) and is at least $16 \mathrm{~km}$ (10 miles) inside waming area 105. Experiment begins.

Second group of simulator subjects are briefed.

First simulator run ends. Subjects are escorted to conference room, debriefed and entertained with a video or reading materials.

Second group of subjects enter simulator.

Second simulator run begins. 
11:10 to

11:30

$11: 40$ to

$12: 00$

$11: 45$ to

$12: 05$

12:05

12:05

$112: 30$

$12: 35$

$12: 50$

13:05

$13: 25$

13:30

13:35

13:40

14:05

$14: 15$

$14: 10$ to

$14: 30$

$14: 35$

14:40
Flight experiment ends.

Aircraft arjives at gate.

Second simulator nun ends.

Box lunches are served to 8 subjects from morning simulator nuns.

Flight subjects who are also simulator subjects are given maps and directions to Bethpage facilities and begin driving their cars. Other flight subjects are paid off and released.

Morning simulator subjects are escorted out through security and given maps and directions to airport.

Morning simulator subjects begin driving to Farmingdale.

Afternoon simulator subjects arrive at security desk.

8 Afternoon simulator subjects are escorted to conference room and served box lunches.

Afternoon flight subjects arrive at boanding lounge for briefing and use of rest rooms.

Third group of simulator subjects are briefed.

Fourth group of simulator subjects remain in conference room to read or watch video.

Subjects enter simulator.

Simulator run begins.

Flight subjects board aircraft.

Aircraft departs gate.

Aircraft takeoff.

Aircraft reaches 4572 meters (15,000 feet) and is at least $16 \mathrm{~km}$ (10 miles) inside waming area 105. Experiment begins.

Fourth group of simulator subjects are briefed.

Third simulator nun ends. Subjects are escorted to conference room, debriefed, paid off and released.

Fourth group of simulator subjects enter simulator.

Fourth simulator $\sin$ begins. 
From the time of takeoff, about 25 minutes were required to climb above 3048 meters ( 10,000 feet) and reach the test area. Each group of subjects then experienced a sequence of roll maneuvers with one of the nine possible combinations of limits on bank angle and roll rate shown in the following table.

Table 3-1. Combinations of limits on bank angles and roll rates.

\begin{tabular}{cccc} 
Max Roll Rate & \multicolumn{3}{c}{ Max Bank Angle } \\
& $14^{\circ}$ & $21^{\circ}$ & $28^{\circ}$ \\
$4^{\circ} \mathrm{sec}$ & $\mathrm{x}$ & $\mathrm{x}$ & $\mathrm{x}$ \\
$6^{\circ} \mathrm{sec}$ & $\mathrm{x}$ & $\mathrm{x}$ & $\mathrm{X}$ \\
$8^{\circ} \mathrm{sec}$ & $\mathrm{x}$ & $\mathrm{x}$ & $\mathrm{x}$
\end{tabular}

For each combination of maximum bank angle and maximum roll rate, there is an implied average speed over the specified right of way. Speed is higher for the more severe combinations of bank and roll rate. $277 \mathrm{~km}$ (172 miles) of the Thruway were simulated, implying trip times ranging from about 40 minutes for the higher limits to about 48 minutes for the most gentle ride.

Appendix $\mathrm{E}$ shows a plot of bank angle versus time for the worst-case trip, i.e. $28^{\circ}$ bank-angle limit with an $8 \% \mathrm{sec}$ roll-rate limit. The unit for the time axis is sec, i.e., the total duration of the test sequence is 2354.4 seconds or 39.23 minutes.

The experimental portion of each flight was conducted in Warning Area 105, southeast of Long Island. Waming Areas are blocks of restricted airspace, which may not be entered without prior authorization from Air Traffic Control. They are normally used for training, military practice missions, and research. Only one aircraft is permitted to occupy a given block of airspace at a time, so that the pilot can devote his full attention to maneuvering without having to watch out for other aircraft.

Direction to the pilot in fying this series of rolls was provided by a computer-driven, simulated attitude display with one bar showing the desired bank angle at each instant and a second showing actual bank angle as measured by a gyro connected to the computer. The pilot's job was simply to keep the bars parallel. The display incorporates additional indicators regarding the desired bank angle 2 seconds and 10 seconds into the future.

The experimenter and flight crew were continuously monitored on radar by Calverton Tracker (Grumman's trackers who normally monitor test and training flights for fighter aircraft). Range and 
bearing information from Calverton VOR were relayed at frequent intervals to one member of the research team who plotted the aircraft's location on a chart. In order to keep the aircraft on a roughly circular course about $100 \mathrm{~km}$ (60 miles) in diameter and well within the boundaries of the waming area, the experimenter inverted the polarity of certain roll maneuvers. Figure 3-6 shows a portion of an aeronautical chart covering the area used for the test flights with the actual plotted positions for one of the flights.

It was agreed in advance that if any subjects reported or displayed symptoms of nausea, the flight would be terminated early. Only one subject did so, on flight \#8, with bank angle limits of $28^{\circ}$ and roll limits of $8 \%$ sec. The pilot immediately began heading back to Republic Airport in a straight line, but after seven minutes of smooth flight, the subject asked that experimental maneuvers be resumed. The last interval of this flight was flown according to plan.

During flight \#7 (28 bank-angle limits and $6^{\circ} / \mathrm{sec}$ roll-rate limits) a second subject vomited, as evidenced by the contents of an air-sickness bag found during cleanup after retuming to Republic Airport. Since that subject never made any indication of illness during the experimental portion of the flight, it is presumed that this incident of emesis occurred just afterward.

At the end of each flight or simulator trip, the research team members collected the rating booklets, debriefed subjects, and recorded any pertinent comments regarding ride quality and comfort. Subjects were queried as to whether they felt dizzy, nauseous, or otherwise unable to drive home safely. Fortunately, the two who experienced vomiting had come with someone else who was not ill and was able to drive them home. Had any been incapacitated, arrangements had been made to transport them home safely by taxicab or other means.

All subjects were paid $\$ 50.00$ at the conclusion of the flight. Extra subjects who are not used were also compensated and rescheduled for a later flight.

Half of the subjects were asked to take a one-hour trip in Grumman's Maglev Simulator located in Bethpage. Subjects who took this extra test were paid an additional $\$ 25$ and given a box lunch between the moming and afternoon test sessions. 
Figure 3-6. Partial aeronautical chart showing the warning area where tests were conducted.

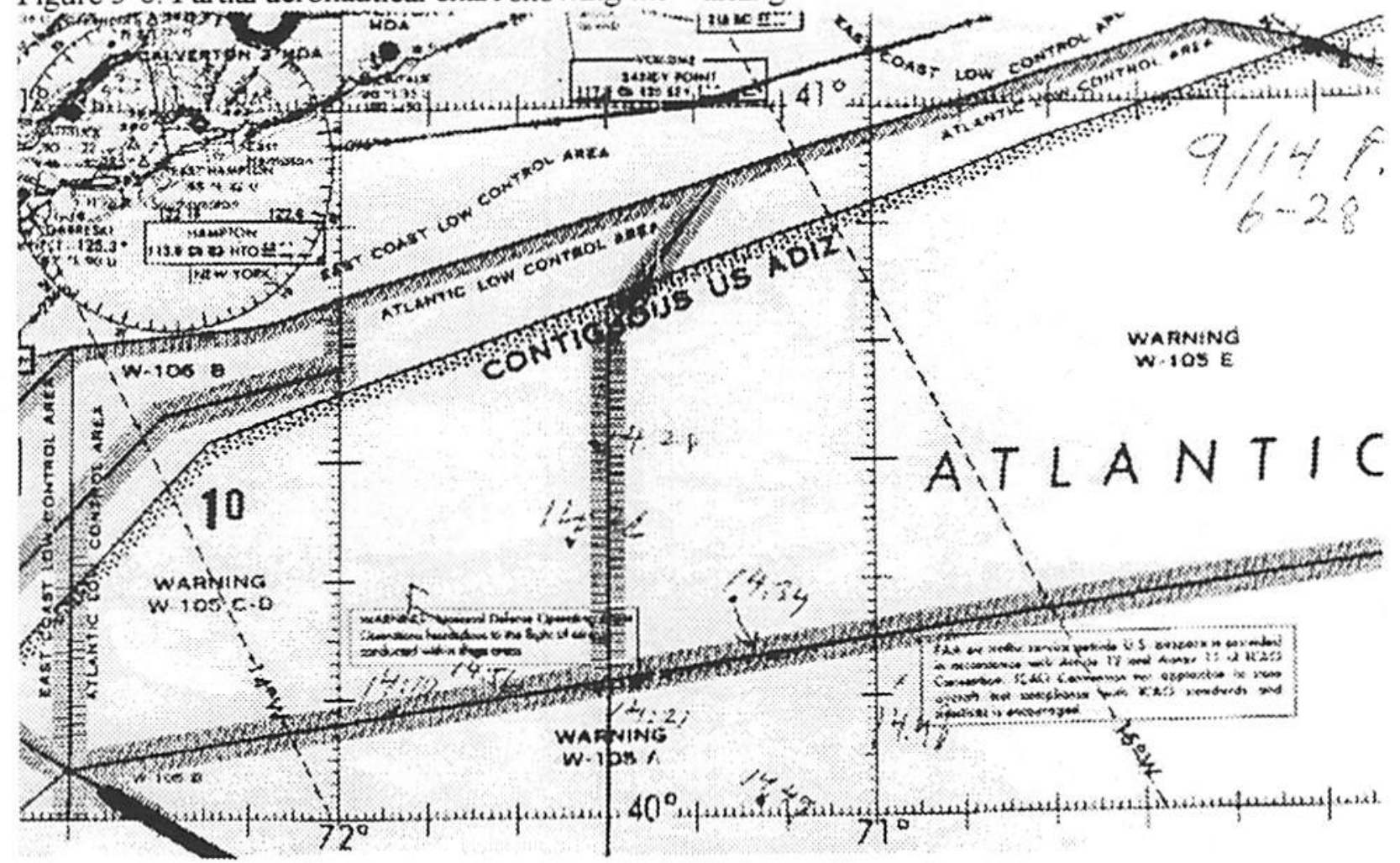




\subsection{Aircraft Instrumentation}

The instrumentation package used for the flight experiments was required to perform three functions:

1. present to the pilot a display showing desired bank angle at each instant in time for a given set of limits.

2. provide feedback to the pilot as to how closely the aircraft's actual bank angle matched the desired bank angle.

3. record the roll rate, actual bank angles and accelerations in all three axes at intervals of 0.1 second.

To present the information on desired and actual bank angles, a small video display was mounted temporarily over the aircraft's normal attitude display. (Figure 3-7) This monitor was driven from a notebook computer running custom software through a converter which transformed the VGA output of the computer into an NTSC video signal for the monitor. The software contained a look-up table with the values for bank angle at 0.2 second intervals and routines to convert these numeric values into inclination angles for a red bar on the display. This computer was linked through its serial port to a second notebook which served as the data-acquisition computer. (Figure 3-8).

The second computer collected readings from three accelerometers and a roll-rate gyro at intervals of 0.1 second. These were installed in a small case located under one of the passenger seats in the aircraft. The accelerometers were contained in an Entran Devices Model EGCS3-A-2 three-axis unit ( $2 \mathrm{~g}$ full scale). The rate gyro was a solid-state device manufactured by Systron-Donner called the GyroChip ${ }^{\mathrm{TM}}$, with a full scale range of plus $/$ minus $20 \%$ sec. The voltage outputs of these transducers were recorded through a Computer Boards Inc. PCM-DAS08 data-acquisition card using Laboratory Technologies' Labtech Notebook ${ }^{\mathrm{TM}}$ sottware. Bank angles were calculated within Notebook ${ }^{\mathrm{TM}}$ by integrating and smoothing the roll-rate data.

Bank angle data were sent across the serial link along with a time stamp, allowing the first computer to update the pilot's display at intervals of 0.2 second. The software also provided two smaller indicators showing what the bank angle would be two seconds ahead of the current moment and the direction of the next maneuver.

This instrumentation allowed pilots to fly a reasonable approximation of the desired sequence of maneuvers with only a couple of hours of practice. Figure 3-9 shows an example of the data acquisition screen displaying the correspondence between desired and actual bank angles. 
Figure 3-7. Attitude display temporarily installed in the cockpit of the Beechcraft 1900C

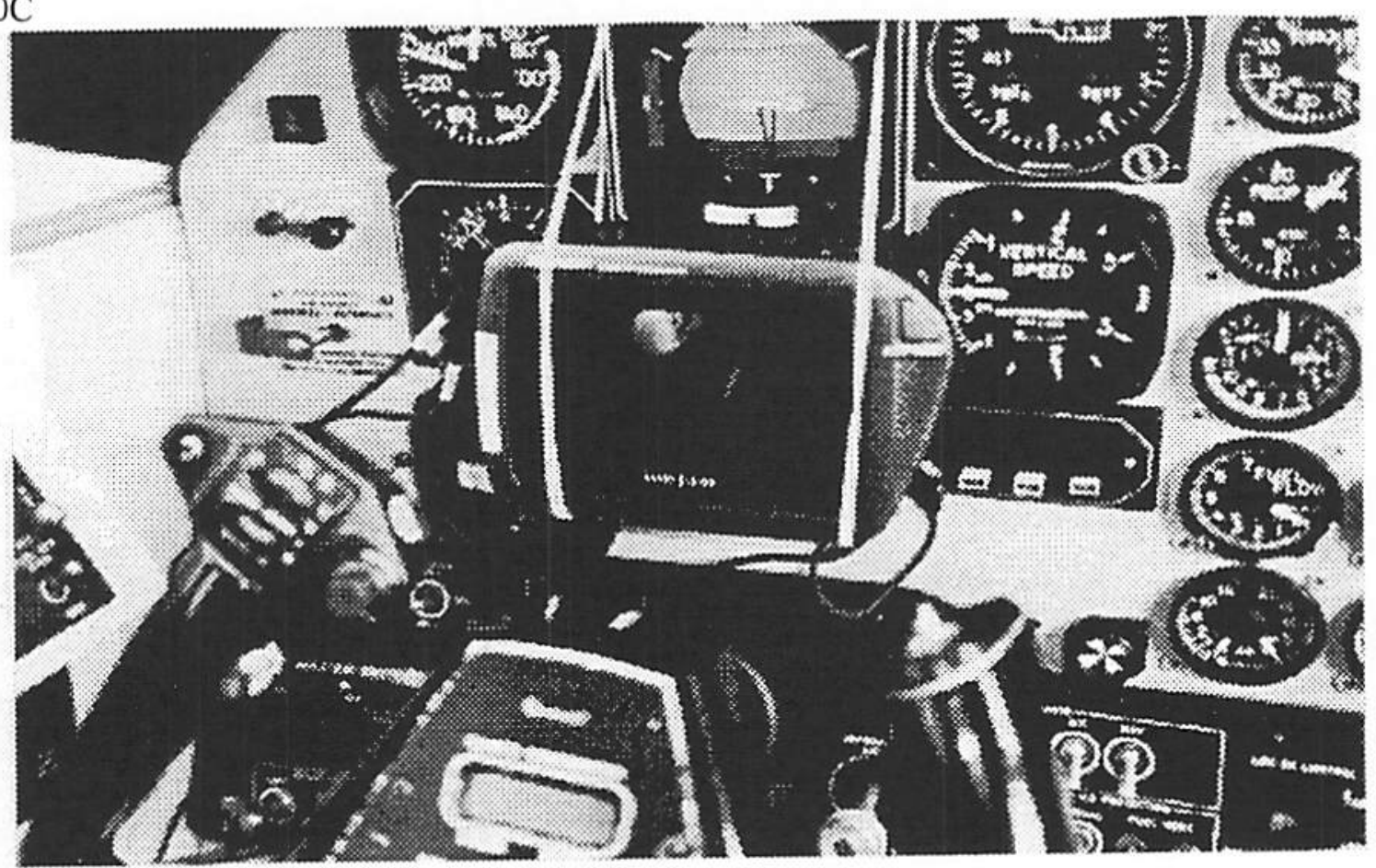

Figure 3-8. Notebook computers used for data acquisition and generation of the cockpit display.

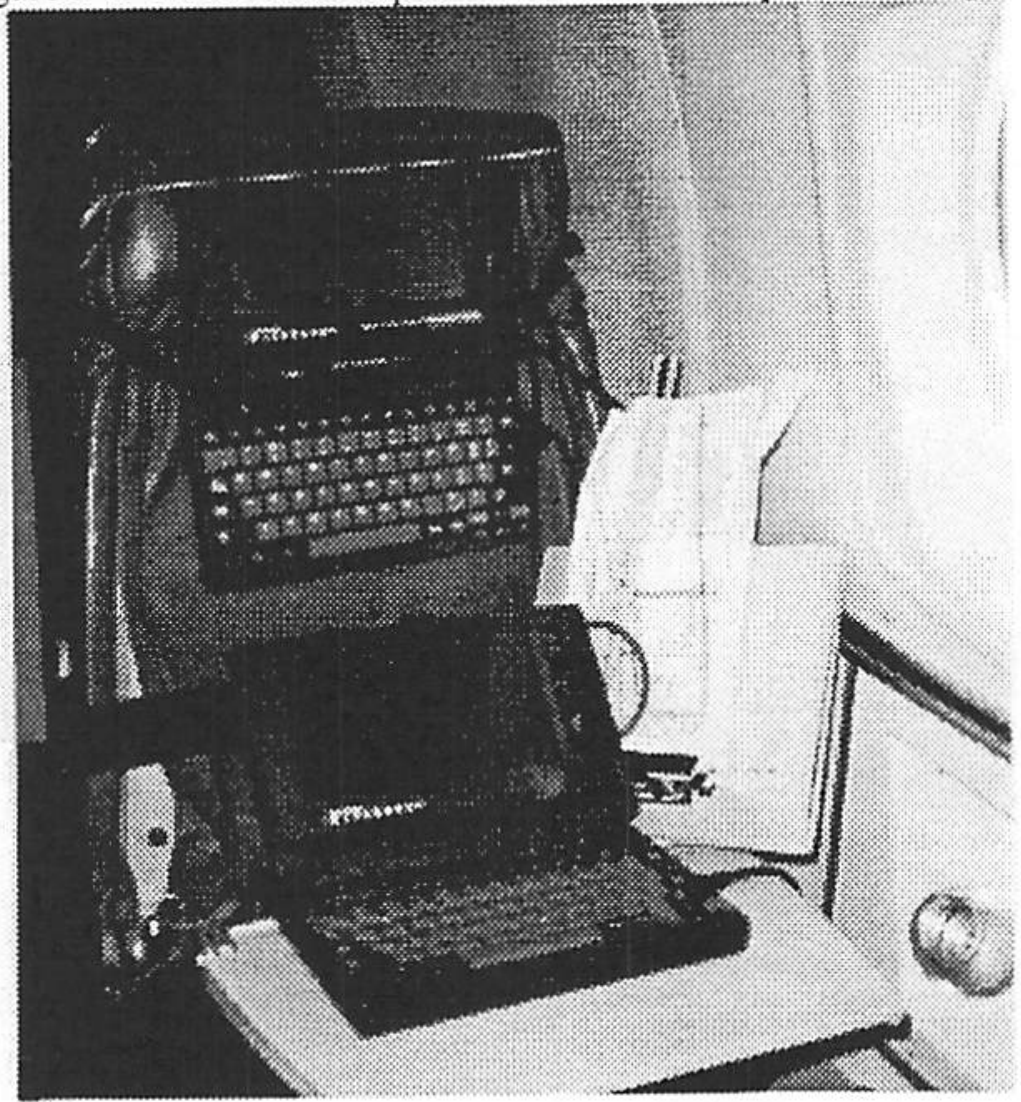


Figure 3-9. Example of the correspondence between the desired bank angles and the actual bank angles during a three-minute period. Desired bank angle (DBA) is shown in black, while actual bank angle (CBA) appears in gray. The deviations in the actual are primarily the results of turbulence and pilot actions in this example.

20.000

DBA
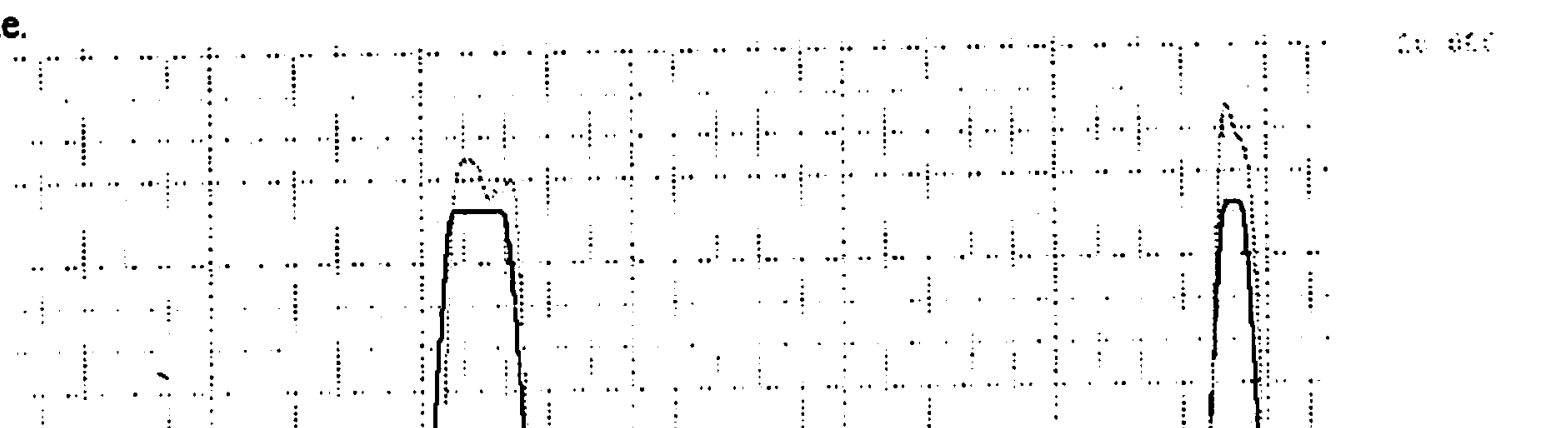

20.00 695.60 SEC(TEF) of $\mathrm{SEC}(\mathrm{TH})$

However the actual vertical acceleration dosage experienced by passengers on the plane trips was significantly greater than the theoretical dosage that should have been accumulated by a Maglev vehicle traversing a guideway built to the nominal limits. This extra vertical acceleration arose from several sources including: (1) turbulence in the atmosphere; (2) altitude changes made in search of smoother air; (3) corrections of drift in the bank-angle measurement instrumentation; (4) extra turns required to keep the aircraft within the restricted air space; and (5) pilot error in following the displayed attitude indication.

The greatest excess of actual MSDV over desired MSDV occurred in Flights 6 and 7, which were characterized by strong northwest winds in the test area and low temperatures. The latter required the use of windshield heaters, which effectively disabled the magnetic compass and eliminated direct feedback to the experimenter as to aircraft heading. 
The peak roll rates were two to four degrees per second higher than intended on each flight. Hence the ratings developed here are conservative. An actual Maglev would not be subject to any of the aforementioned sources of vertical acceleration, and would generate substantially less vertical acceleration in the 0.06 to $0.5 \mathrm{~Hz}$ range than the plane flights did. Thus the incidence of motion sickness observed ( $8 \%$ of the subjects reported "Intermittently nauseous" or worse) probably exceeds that which would occur aboard an actual Maglev system built to the same nominal limits for bank angle and roll rate.

\subsection{Simulator Instrumentation}

Since the simulator could reproduce a specified series of movements precisely and consistently, there was no need to record accelerometer and rate gyro data in every trial. Rather, the simulator was programmed by Northrop Gumman staff to one of the nine possible combinations of roll-rate and bank-angle limits for each trial using a disk of bank-angle values by time at 0.1 second intervals as supplied by the Volpe Center. These bank angles were reproduced exactly in the simulated out-thewindow view, but the actual roll of the simulator capsule was limited to about one-third of the specified value at in order not to generate unpleasant lateral accelerations.

Simulated trips of $80 \mathrm{~km}$ (50 miles) each were recorded on the same instrumentation as was used in the airplane for the following limits:

1. $14^{\circ}$ bank angle and $4^{\circ} / \mathrm{sec}$ roll rate

2. $14^{\circ}$ bank angle and $8 \% \mathrm{sec}$ roll rate

3. $28^{\circ}$ bank angle and $4 \% \mathrm{sec}$ roll rate

4. $28^{\circ}$ bank angle and $8 \% \mathrm{sec}$ roll rate.

Figure 4-14 contains an example of the data from the accelerometers and roll-rate gyro for the fourth case. 


\section{Analysis of Data}

\subsection{Description of motion-sickness dosage value}

A Maglev vehicle traveling at high speed and negotiating frequent curves requiring bank angles greater than $20^{\circ}$ has a potential for inducing motion sickness in some segment of the passenger population. If the route alignment and speed are known at all points on the route then the complete set of passenger motions are readily available. For an assumed hypothetical route alignment, one can determine a minimum-time trajectory given limits on the speed, acceleration, deceleration, bank angle and roll rate. Such a trajectory was calculated for the New York state Thruway data and was described in Section 3.1.

With regard to motion sickness, vertical accelerations at frequencies of 0.1 to $0.5 \mathrm{~Hz}$ are the predominant source of motion sickness, although other motions and visual stimuli can contribute. The known facts are well summarized in Ref. 5 and Ref. 8 and are reflected in ISO standard 2631 for ride quality measurements. Griffin and coworkers have unified much previous work. They have proposed a dosage measure for motion sickness which is the time integral of the square of the frequency-weighted vertical acceleration. This means that the vertical acceleration as a time series is to serve as input to a filter specified in British Standard 6841 (Ref 8) The output is the frequency-weighted acceleration. It has frequencies appreciably outside the 0.06 to $0.5 \mathrm{~Hz}$ band significantly attenuated. The cumulative measure specified in the British Standard is referred to there as the Motion Sickness Dosage Value, which we refer to also as the MSDV. (See Appendix C , KINCALC.SAS for discussion of a method for calculating MSDV).

The dose measure was also used in selecting trajectories as scenarios for the experiment which were not so rigorous as to be likely to induce vomiting in many passengers yet not so mild as to fail to induce any significant level of discomfort in any significant proportion of the persons evaluating the ride. The former limiting case could force flights to be cut short, while the latter would mean that no useful data were obtained.

There is a further potential use for a properly validated and calibrated Motion Sickness Dosage Value. Just as optimum trajectories can be derived maximizing average speed with acceleration, bank angle and roll rate limited, we could add one more constraint: that total (cumulative) MSDV be limited to a certain value. Such analysis would provide the best analytic procedure for finding a velocity profile which allows maximum average speed while not inducing motion sickness in the passengers. Because we can in principle calculate the MSDV for every conceivable trajectory it is possible to determine where to go fast and where to go slow in order to hold down the MSDV. The filter output itself indicates where the incremental dosage is high and these places are where speed should probably held down. Regardless of the computational procedure the goal is easily stated in principle: 
If one were to calculate the total MSDV for each trajectory satisfying the basic constraints (acceleration, bank angle, roll rate), which would have the highest average speed of all those which satisfy the motion sickness dosage limit constraint? With modern optimization techniques, one need not examine even approximately all feasible trajectories and the calculational procedure will probably be easily within modest computing resources.

What does the dosage measure say about the proposed trajectories for the NY thruway route? Since vertical acceleration vs time (ignoring grade changes) is available one can calculate the cumulative Motion Sickness Dosage Index for each of nine trajectories that have been developed. These nine cases represent all possible combinations of 3 levels each of bank angle and roll rate. These are as follows:

$\begin{array}{cccc} & \text { Low } & \text { Medium } & \text { High } \\ \text { Bank Angle } & 14^{\circ} & 21^{\circ} & 28^{\circ} \\ \text { Roll Rate } & 4^{\circ} / \mathrm{sec} & 6^{\circ} / \mathrm{sec} & 8^{\circ} / \mathrm{sec}\end{array}$

The trajectories are constructed to maximize average speed over the whole route while holding acceleration/deceleration, bank angle, and roll rates to within the given limits. Each trajectory has (in general) a different overall average speed. In general the less restrictive the constraints the higher the average speed.

Some preliminary results concerning the nine test trajectories representing the New York State Thruway route can be given. Table 4-1 presents results on trajectories for all nine combinations of the conditions shown above. For each trajectory the values of the two conditions (independent variables) are given. Also, given are the outcomes variables: average speed (in $\mathrm{kph}$ and $\mathrm{mph}$ ) and MSDV (cumulative over the whole route) calculated in two ways.

Table 4-1. Characteristics of the nine test trajectories: Bank angle, roll rates, MSDVz (actual and desired), average speed and transit time for $277 \mathrm{~km}$ (172 miles) of the New York State Thruway.

\begin{tabular}{|c|c|c|c|c|c|c|c|}
\hline \multirow{2}{*}{$\begin{array}{c}\text { Flight } \\
\#\end{array}$} & \multirow{2}{*}{$\begin{array}{l}\text { Roll } \\
\text { Rate }\end{array}$} & \multirow{2}{*}{$\begin{array}{l}\text { Bank } \\
\text { Angle }\end{array}$} & \multirow{2}{*}{$\begin{array}{c}\text { MSDVz } \\
\text { Desired }\end{array}$} & \multirow{2}{*}{$\begin{array}{c}\text { MSDVz } \\
\text { Actual }\end{array}$} & \multicolumn{2}{|c|}{ Average Speed } & \multirow{2}{*}{$\begin{array}{c}\text { Transit } \\
\text { Time } \\
\text { (min.) }\end{array}$} \\
\hline & & & & & $(\mathrm{kph})$ & (mph) & \\
\hline 1 & 4 & $\overline{21}$ & 2.1 & $\overline{3.1}$ & 341 & 212 & 48.6 \\
\hline 2 & 4 & 14 & 1.6 & 3.6 & 328 & 204 & 50.5 \\
\hline 3 & 4 & 28 & 1.9 & 2.9 & 341 & 212 & 48.6 \\
\hline 4 & 6 & 21 & 3.5 & 6.0 & 378 & 235 & 44.0 \\
\hline 5 & 6 & 14 & 1.9 & 2.4 & 336 & 209 & 49.5 \\
\hline 6 & 8 & 14 & 1.9 & 4.6 & 336 & 209 & 49.5 \\
\hline 7 & 6 & 28 & 4.0 & 7.5 & 383 & 238 & 43.3 \\
\hline 8 & 8 & 28 & 5.7 & 5.8 & 410 & 255 & 40.5 \\
\hline 9 & 8 & 21 & 4.2 & 4.8 & 389 & 242 & 42.6 \\
\hline
\end{tabular}


MSDVz and average speed were calculated for 27 different combinations of limits on roll rate, bank angle and longitudinal acceleration for the New York State route. Dosage vs. average speed is given in Figure 4-1. Note that MSDVz is determined largely but not solely by average speed.

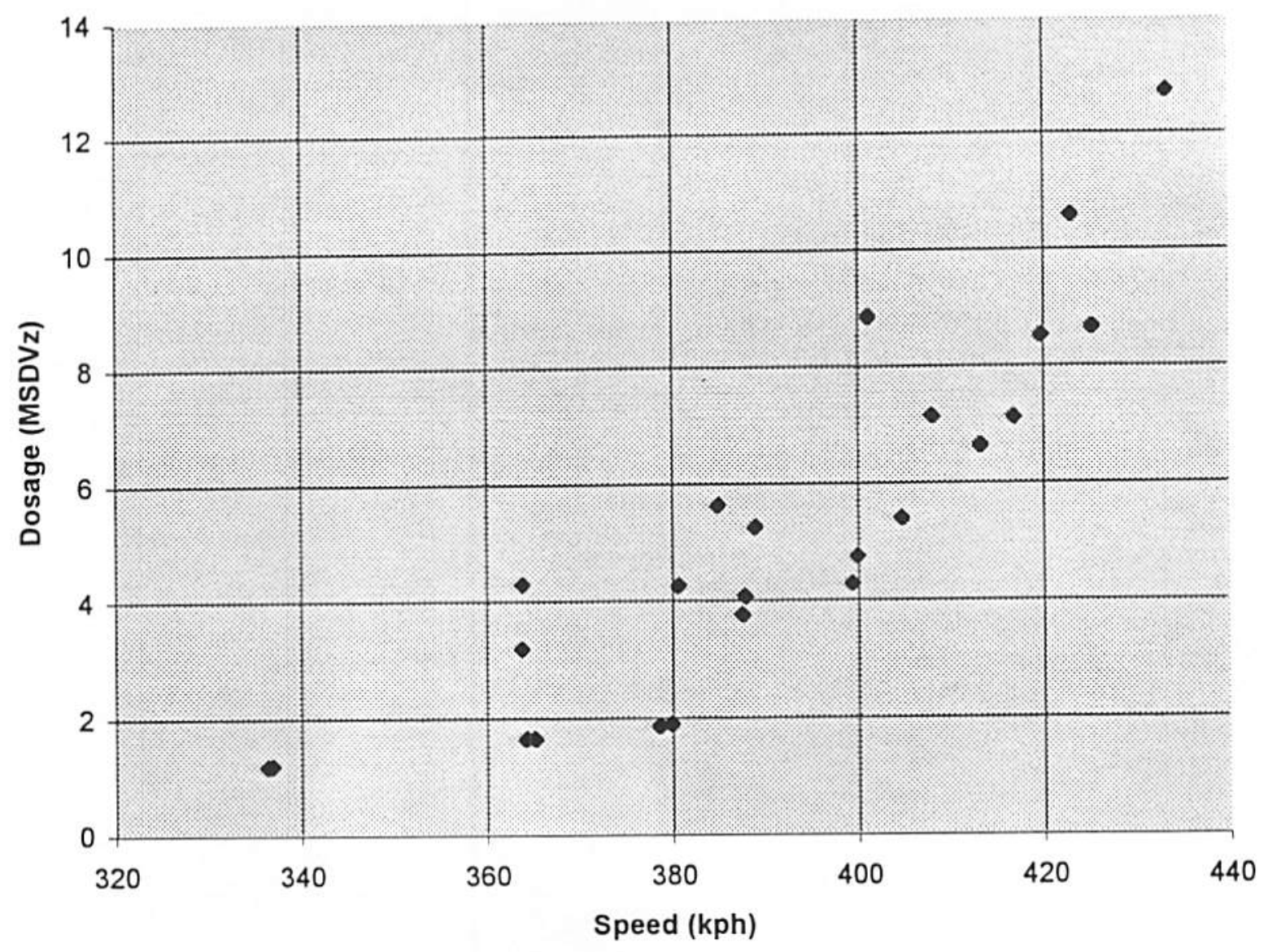

Figure 4-1. Motion-sickness dosage for 27 hypothetical combinations of bank-angle and roll-rate limits for the New York State Thruway route. 


\subsection{Analysis of Flight Data}

As noted in Table 4-1 above, all of the flights produced more vertical acceleration than would have been caused solely by following the hypothetical Maglev trajectory. The excess dosage ranged from about $2 \%$ on Flight 8 to more than $100 \%$ on Flights 2 and 6 . These excess dosages were the result of a variety of problems discussed in Section 3.6. Thus all of the analyses and findings which follow are conservative, i.e. a real Maglev following the same trajectory should produce less passenger discomfort and motion-sickness.

On all but two of the flights, about four out of five passengers rated ride quality as "somewhat comfortable" or better in every interval. For the other two flights the proportion of such ratings fell to about two out of three Figure 4-2 shows these data presented in terms of the percentage of subjects who reported a rating of "heutral" or worse in any interval of a specified flight.

\section{Percentage of passengers reporting "neutral" or worse}

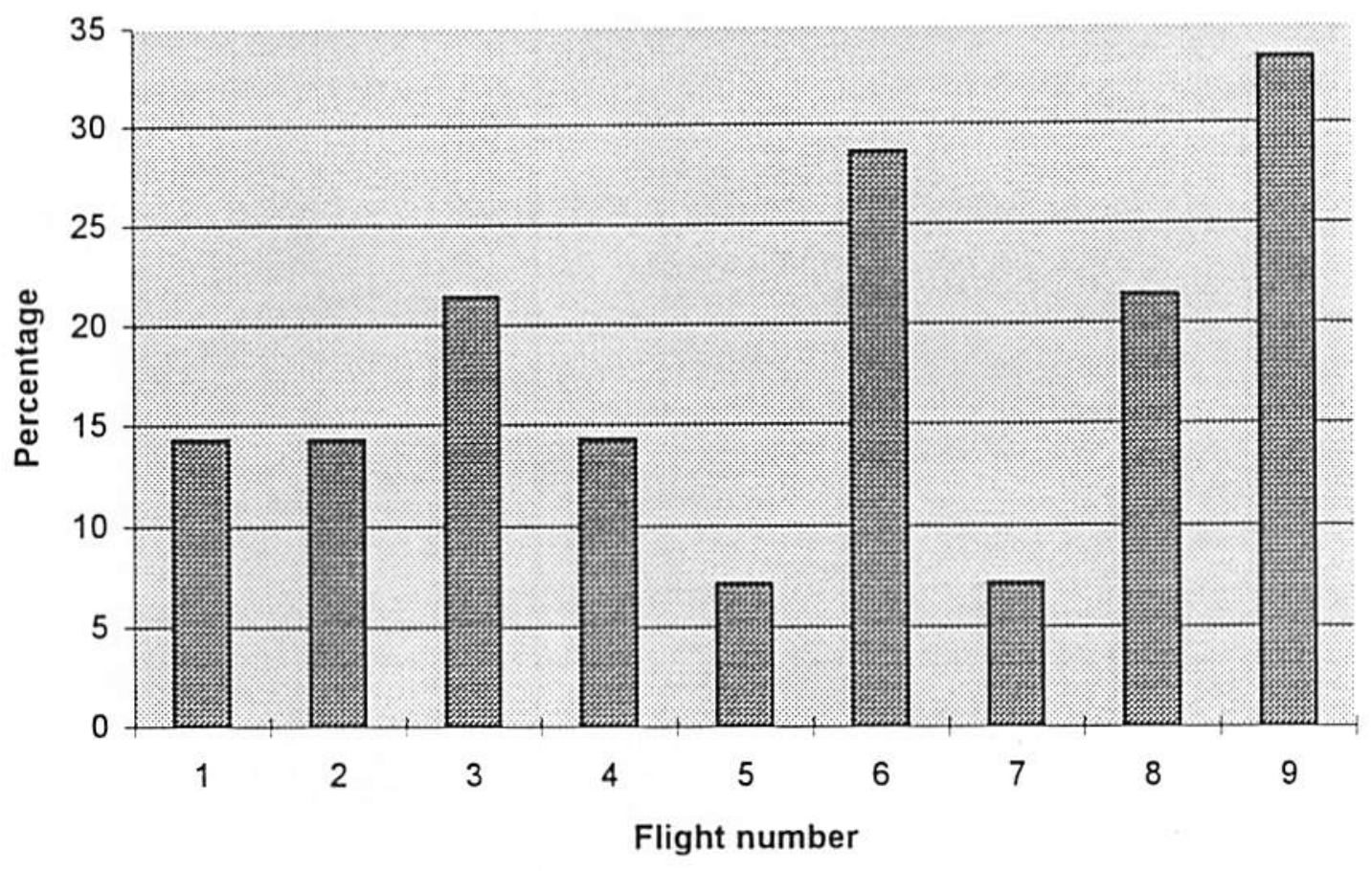

Figure 4-2. Passenger ratings of ride comfort during the flight experiments as "heutral" or worse (4 or greater on the 7-point scale).

By the more rigorous standard of "comfortable" or better, only about half the passengers on most flights were that well pleased. Note in Figure 4-3 that on flights 5 and 7, almost everyone felt 
comfortable. Flight 5 had the lowest actual MSDVz, but flight 7 had the highest. The authors can only conclude that differences between subjects as to how ride comfort is perceived overwhelmed the actual differences in ride motions.

\section{Percentage of passengers reporting "somewhat comfortable" or worse}

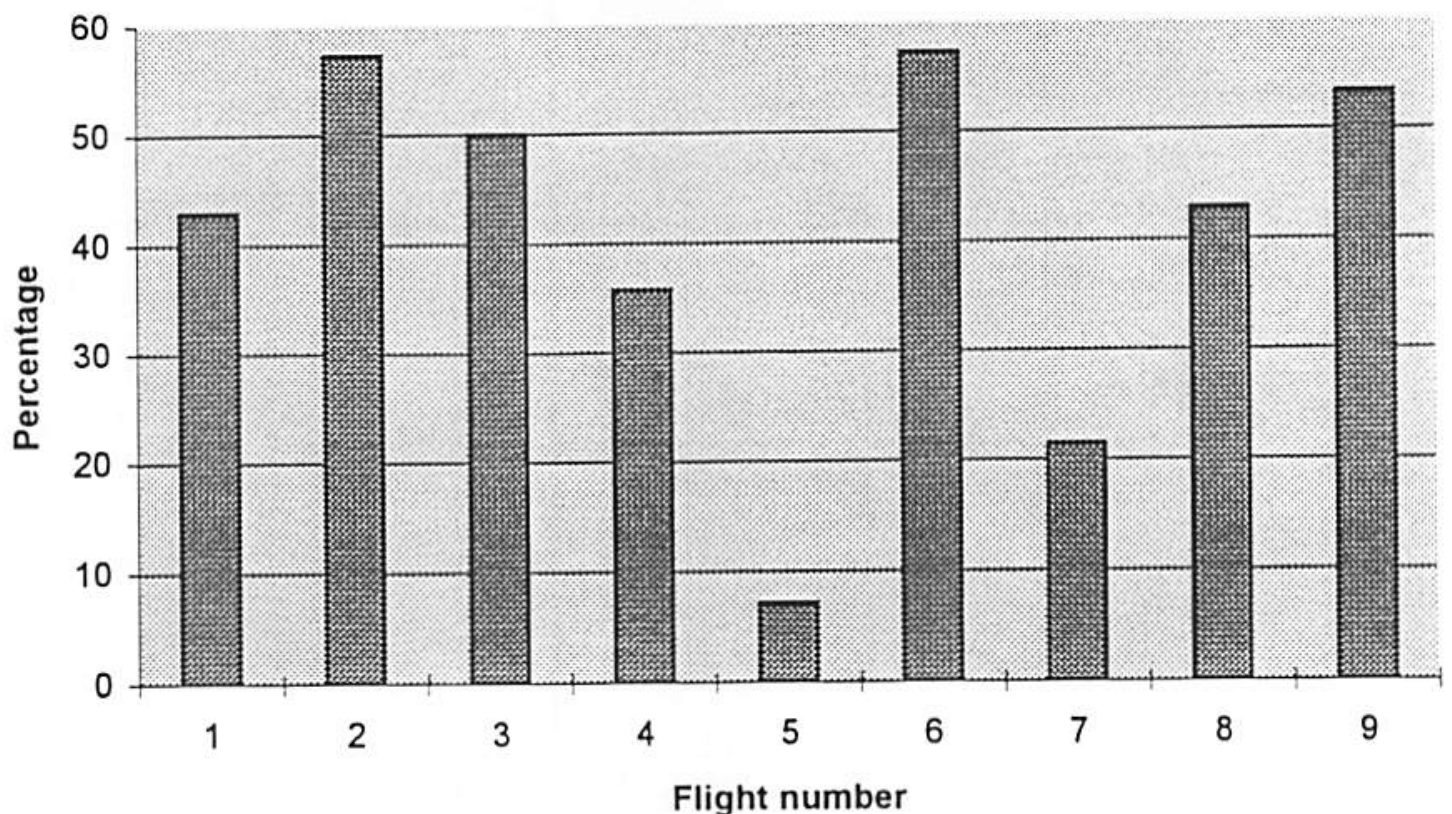

Figure 4-3. Passenger ratings of ride comfort during the flight experiments as "somewhat comfortable" or worse (3 or greater on the 7-point scale).

Although only two subjects actually vomited, all of the flights induced some queasiness in two or more passengers as indicated in Figure 4-4. 


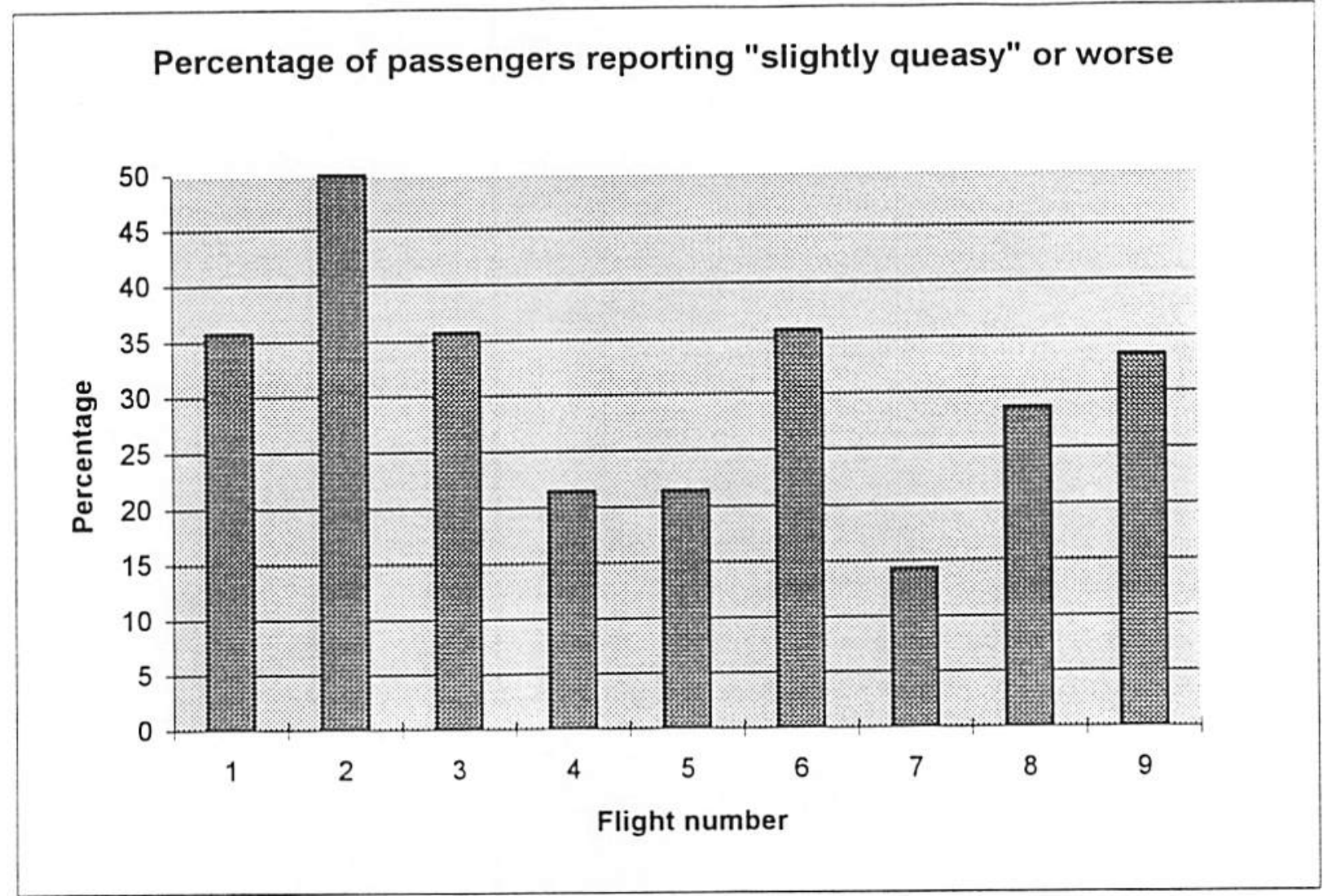

Figure 4-4. Passengers reporting "slight queasiness" or worse (3 or greater on the 7-point scale).

That such substantial percentages of the subjects should have felt queasy or worse is hardly surprising in view of the substantial dose of vertical acceleration they received. Figure 4-5 shows the roll rates and accelerations in all three axes for Flight 8 , which had nominal limits of $8 \% \mathrm{sec}$ and $28^{\circ}$ maximum bank angle. 


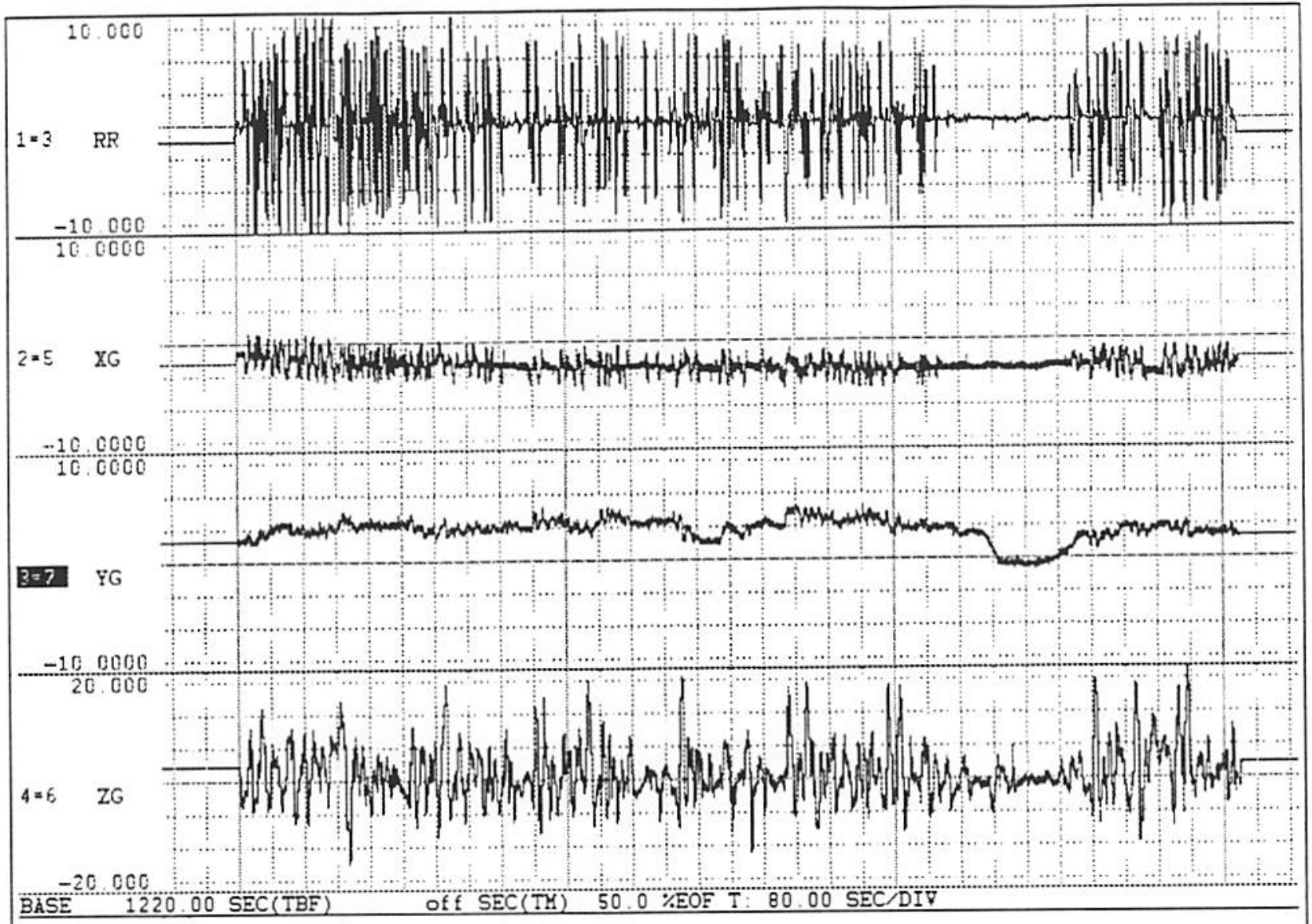

Figure 4-5. Roll rates and accelerations for Flight 8 . Note period of about five minutes near end of flight in which traces are nearly flat and typical of normal airliner conditions. This occurred after a subject had vomited. After a few minutes, that subject requested that the experiment be resumed.

The spectrum of the vertical acceleration record shown above is presented in Figure 4-6, which shows that most of the energy in the vertical movements is found below one $\mathrm{Hz}$, with the peak at $0.0345 \mathrm{~Hz}$. This implies that peak power is associated with roll maneuvers with periods of about 29 seconds.

Power spectra for the other flights are similar in shape with their peaks at nearly the same frequency. Peak amplitudes vary by several $\mathrm{dB}$, depending on the severity of the ride. 


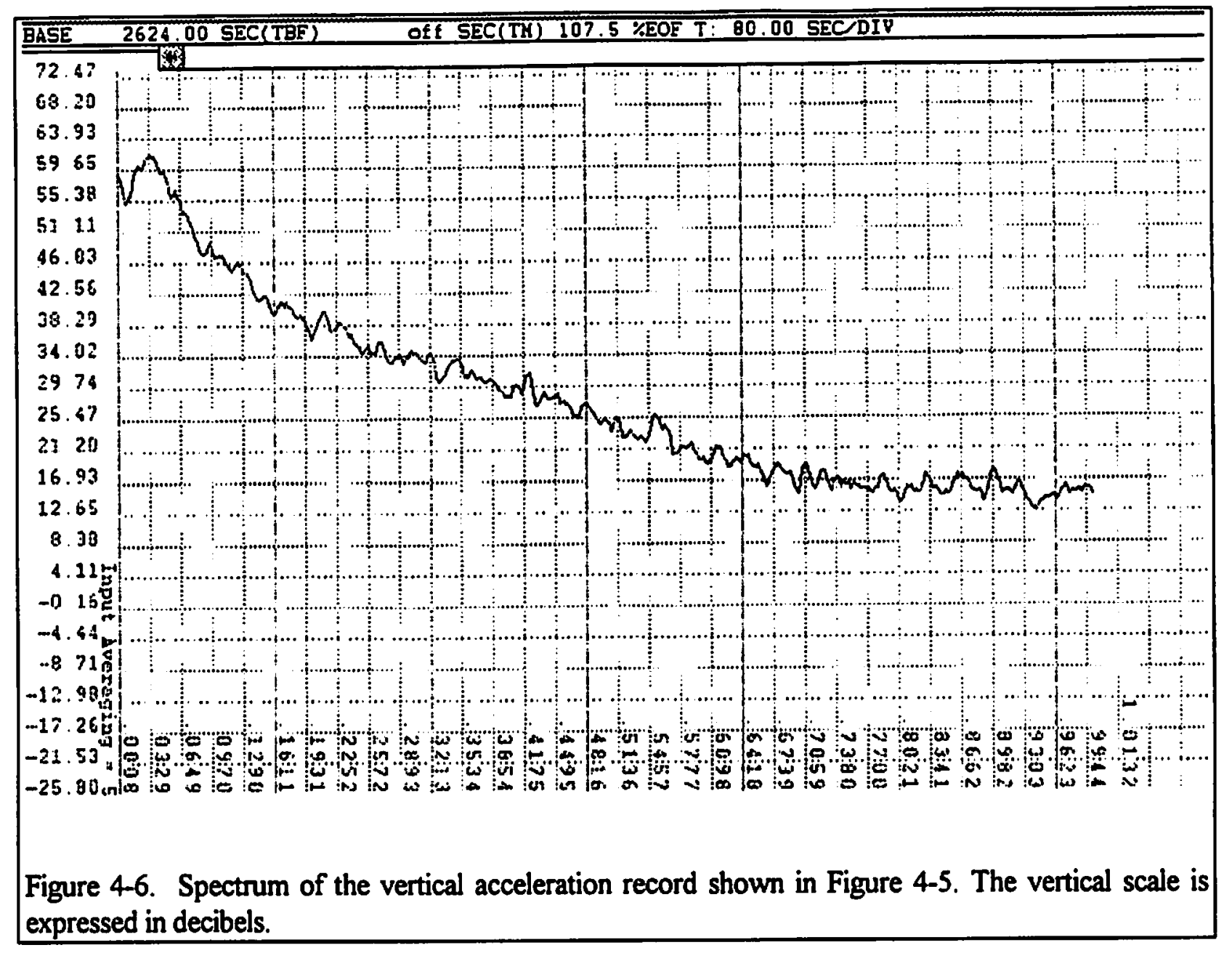

\subsection{Discussion of lack of correlation between MSDV \& subject ratings}

The two dependent variables of interest are the motion sickness ratings (scale: 1 to 7) and ride comfort ratings (scale: 1 to 7). There were 635 responses on each scale ( 9 flights times 14 subjects per flight times 5 ride intervals per flight equals 630, plus five more from an extra subject carried on Flight 8). Figures 4-7 and 4-8 below show histograms of the motion sickness and ride comfort ratings respectively. The figures show that the vast majority of subjects were comfortable and free of motion sickness. Uncomfortable ratings for ride quality were reported about twice as frequently as those of motion sickness. 


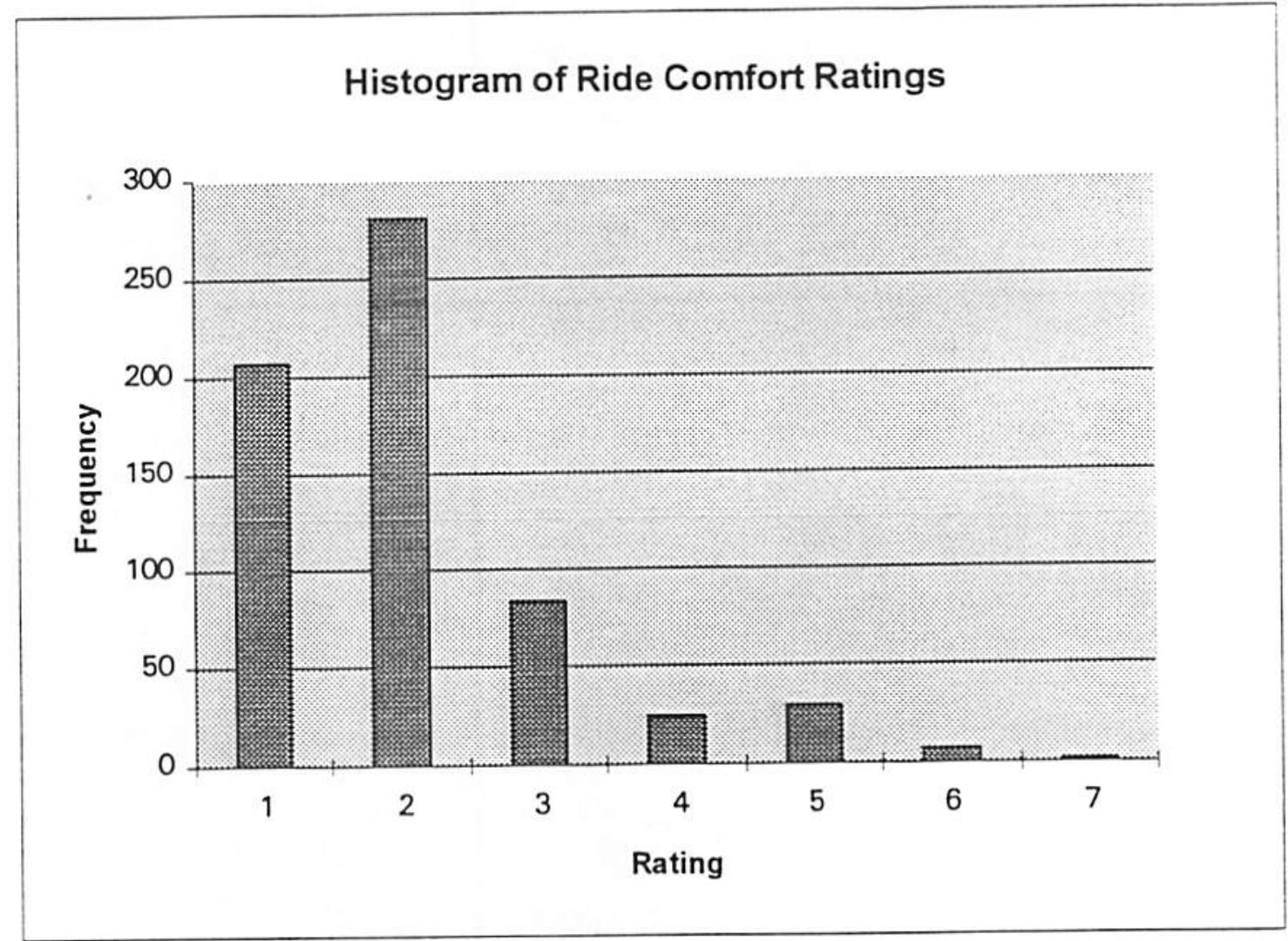

Figure 4-7. Passenger ratings of ride comfort summed across all flights(1=very comfortable, $7=$ very uncomfortable). 


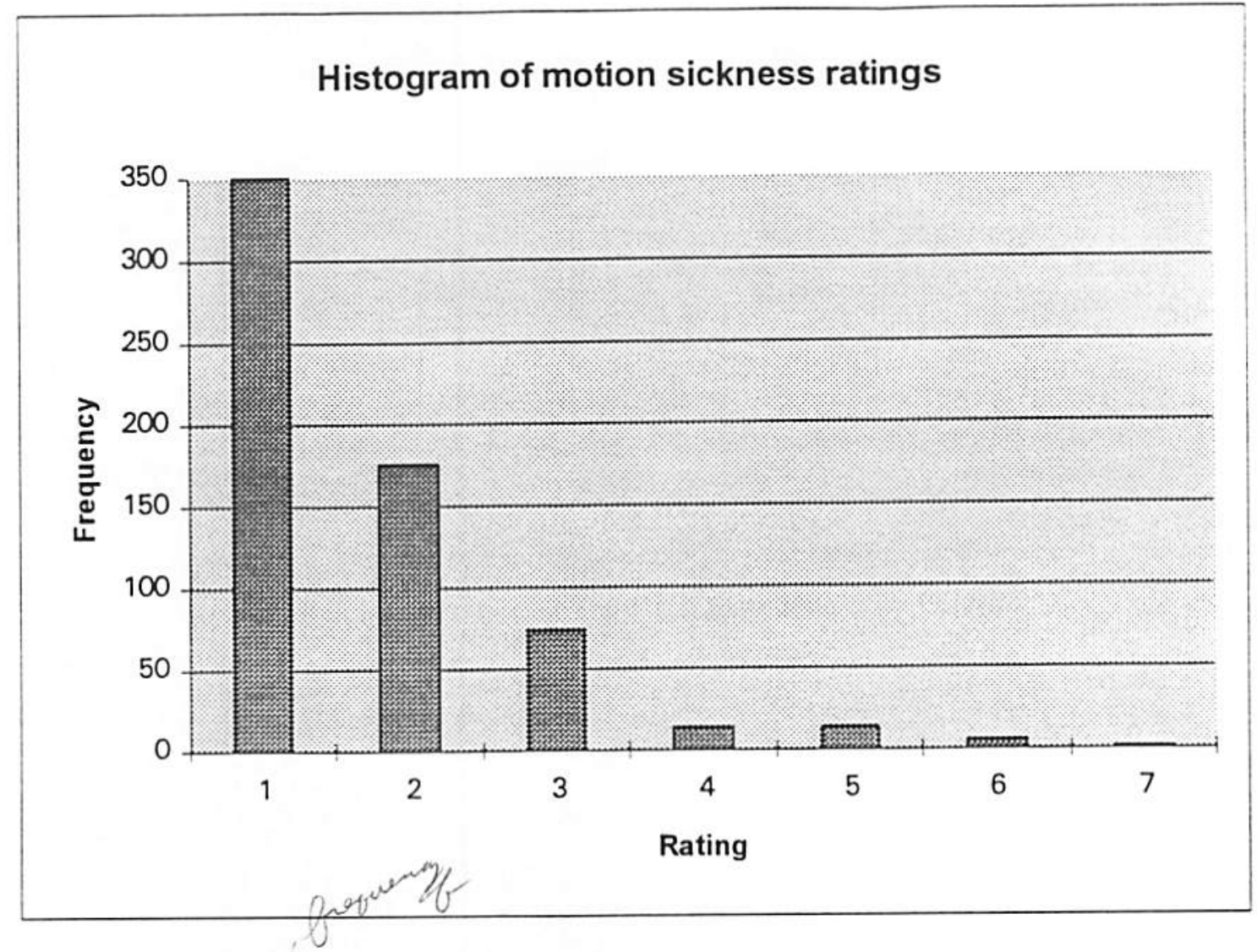

Figure 4-8. Passenger ratings of motion sickness summed across all flights.

Of primary concern is the extent to which the variance in the two dependent variables can be explained by variation in the ride parameters. There were three parameters which have been examined: Motion Sickness Dose Value (MSDVz or Dose), mean square of roll rate (Roll), and mean square vertical acceleration (MSYG). Dose is calculated as a frequency-weighted average of the vertical acceleration as measured by the accelerometers. Mean square of roll rate involves averaging the squared roll-rate values measured by the rate gyro over the relevant time interval. Mean square vertical acceleration, like the Dose value, is based on vertical accelerations measured by the accelerometer, but unlike the Dose value does not involve frequency weighting. Each of these measurements is taken in two forms: a "local" form and a "cumulative" form. In the local form only the measurements from the relevant ride interval are included. The "cumulative" form includes all measurements from the start of interval 1 for the given flight.

Table 4-2 displays the results of fitting two linear models to the motion sickness data. The first model (model 11) includes subject and cumulative dose as independent variables. The second includes the aforementioned plus the flight interval variable. One interesting result is that the motion sickness variable is a function of the flight interval; the sickness increases as the flight continues (note that the parameter estimate for interval is positive). This agrees with previous research which indicates that duration of exposure to nauseogenic motions elevates a person's motion sickness levels. Thus any 
regression with a cumulative motion measure, such as cumulative dose, reveals a significant relation between the cumulative measure and the motion sickness value, as evidenced in Model 11. On the other hand, since such a relationship might be explained as an artifact of the "duration of exposure" / motion sickness relation, it is important to consider the additional explanatory power of the proposed motion variable in a model which already includes interval. Only by demonstrating such an effect can we conclude that the motions measured by the motion variable are contributing to the elevated motion sickness scores. Examination of Model 12 in Table 4-2 shows that this effect was not significant. 
Table 4-2. Comparison of two linear models fitted to the motion sickness ratings.

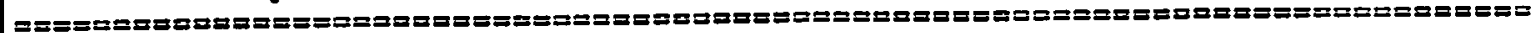
General Linear Models Procedure Dependent Variable: MDT_SICK

Source

Model

Error

Corrected Total

Source
SUBJ

COMDOSE

Parameter

INTERCEPT

CUMDOSE

$\begin{array}{rr}\text { DF } & \begin{array}{r}\text { Sum of } \\ \text { Squares }\end{array} \\ 127 & 531.950487 \\ 505 & 112.981582 \\ 632 & 644.932070 \\ \text { C.V. } \\ \text { care } & 27.69722\end{array}$

0.824816

Model 11

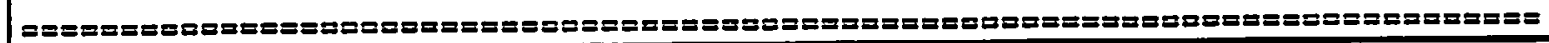

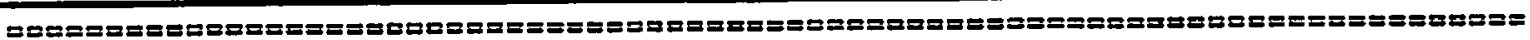
Model 12

General Linear Models Procedure

Dependent Variable: MOT_SICK

\section{Source}

Model

Error

Corrected Total

128
504
632
$R-S q u a r e$
0.827295

DF

128

632

0.827295

$\begin{array}{lrrrrr}\text { Source } & \text { DF } & \text { Type III SS } & \text { Mean Square } & \text { F Value } & \text { Pr > F } \\ \text { SUBJ } & 126 & 524.554009 & 4.163127 & 18.84 & 0.0001 \\ \text { INITERV } & 1 & 1.598802 & 1.598802 & 7.23 & 0.0074 \\ \text { CUMDOSE } & 1 & 0.001014 & 0.001014 & 0.00 & 0.9460\end{array}$

\begin{abstract}
Sum of
Squares

533.549289

111.382781

644.932070
\end{abstract}

c.v.

27.52782
Mean Square

4.205458

7.368418
F value $\quad P r>F$

$18.72 \quad 0.0001$

4.188587

0.223726

ROOt MSE

0.47300

$F$ value $\quad \operatorname{Pr}>F$

$\begin{array}{ll}18.80 & 0.0001\end{array}$

$32.94 \quad 0.0001$

\begin{tabular}{|c|c|c|c|c|}
\hline | Parameter & Estimate & $\begin{array}{c}\text { T for Ho: } \\
\text { Parameter }=0\end{array}$ & $\operatorname{Pr}>|T|$ & $\begin{array}{c}\text { Std Error of } \\
\text { Estimate }\end{array}$ \\
\hline $\begin{array}{l}\text { INTERCEPT } \\
\text { INTERV } \\
\text { COMDOSE }\end{array}$ & $\begin{array}{r}1.554682580 \mathrm{~B} \\
0.086166335 \\
-0.003465503\end{array}$ & $\begin{array}{r}6.50 \\
2.69 \\
-0.07\end{array}$ & $\begin{array}{l}0.0001 \\
0.0074 \\
0.9460\end{array}$ & $\begin{array}{l}0.23923649 \\
0.03203569 \\
0.05116332\end{array}$ \\
\hline
\end{tabular}

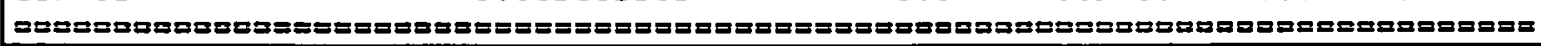


Another question involves the relationship of the flight motion measures to the subjective measures on a flight by flight basis. Specifically, did those flights which experienced the greatest motions produce the greatest degree of motion sickness and discomfort? The answer to this question appears to be "NO". Table 4-3 shows the total of 3 motion variables (MSDVz, mean square vertical accelerations, and mean square roll rates) as well as 4 summary measures of the motion sickness and comfort ratings (average of the average and average of the maximum motion sickness and ride comfort). Table 4-4 is the correlation matrix of the 7 variables presented in Table 4-3. Note that this analysis treats each flight as producing one observation; thus we have nine observations in the data set. This small number of observations, combined with the high degree of subject variability, may be partially responsible for the lack of a relationship between the motion variables and the response variables. It may also be due to the range of motions included in the study. Another surprising finding in the correlation matrix is the low correlation $(r=125)$ between the MSDVz and the mean square vertical accelerations. The key difference between these two measures is that the MSDVz weights low frequencies (from 0.06 to 0.5 $\mathrm{Hz}$ ) heavily and weights other frequencies zero, while the MS Accel does not use any frequency weighting.

Table 4-3. Dosages and subject responses for the nine flights.

Abbreviations used in this table are as follows:

Total MSDVz - Cumulative motion-sickness dosage value

MS Accel - Cumulative (unweighted) vertical acceleration

MS Roll - Cumulative mean square of roll rate

AMMS - Average (per flight) of the maximum (per person) motion-sickness rating

AMRC - Average (per flight) of the maximum (per person) ride-comfort rating

AAMS - Average (per flight) of the average (per person) motion-sickness rating

AARC - Average (per flight) of the average (per person) ride-comfort rating.

\begin{tabular}{|c|ccccccc|}
\hline Flight & Total MSDVz & MS Accel & MS Roll & AMMS AMRC AAMS AARC \\
\hline 1 & 3.1 & 39.3 & 5.0 & 1.9 & 2.4 & 1.5 & 1.8 \\
2 & 3.6 & 22.9 & 4.3 & 2.6 & 2.7 & 2.0 & 2.3 \\
3 & 2.9 & 24.0 & 4.4 & 2.0 & 2.8 & 1.7 & 2.2 \\
4 & 6.0 & 22.0 & 11.1 & 2.0 & 2.5 & 1.5 & 2.1 \\
5 & 2.4 & 9.5 & 5.1 & 1.6 & 1.9 & 1.3 & 1.5 \\
6 & 4.6 & 11.2 & 5.5 & 2.6 & 3.2 & 2.2 & 2.5 \\
7 & 7.5 & 28.0 & 10.5 & 1.6 & 2.1 & 1.4 & 1.7 \\
8 & 5.8 & 23.4 & 11.3 & 2.1 & 2.7 & 1.7 & 2.0 \\
9 & 4.8 & 16.1 & 9.5 & 2.3 & 3.1 & 2.1 & 2.6 \\
\hline
\end{tabular}


Table 4-4. Correlation matrix for dosages and subject responses.

\begin{tabular}{lrrrrrrr}
\hline & \multicolumn{1}{c}{ Total } & MS Accel & MS Roll & AMMS & AMRC & AAMS & AARC \\
& \multicolumn{1}{c}{ MSDVz } & & & & & & \\
\hline Total MSDVz & 1 & & & & & & \\
MS Accel & 0.125 & 1 & & & & & \\
MS Roll & 0.866 & 0.051 & 1 & & & \\
AMMS & -0.077 & -0.266 & -0.208 & 1 & 1 & \\
AMRC & -0.013 & -0.219 & -0.052 & 0.872 & 0.933 & 1 \\
AAMS & 0.000 & -0.320 & -0.139 & 0.959 & & \\
AARC & 0.029 & -0.258 & -0.008 & 0.859 & 0.960 & 0.922 & \\
\hline
\end{tabular}

Figure 4-9 shows the mean motion sickness rating for each interval on each flight (45 points). The lack of correlation with the cumulative dosage value is evident in the scatter.

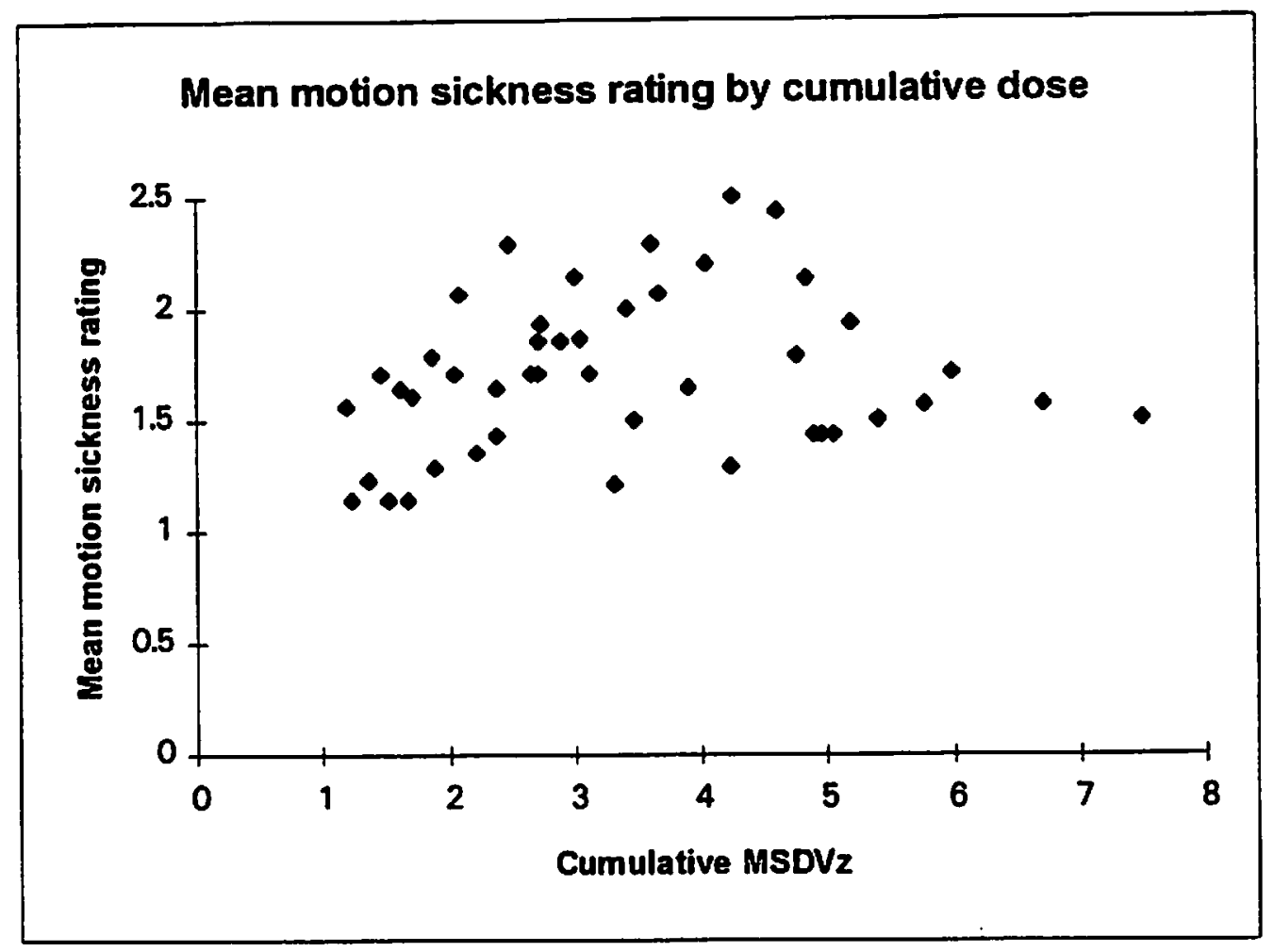

Figure 4-9. Motion sickness ratings by cumulative dose.

Figure 4-10 is a scatter plot of the 635 individual subject ratings. Since there were nine flights with five ratings each, there are only 45 discrete values which occur on the horizontal scale. Subjects were constrained to one of seven integer values for each response, so that most points on this scatter plot represent more than one subject response, i.e. there are many hidden points. 


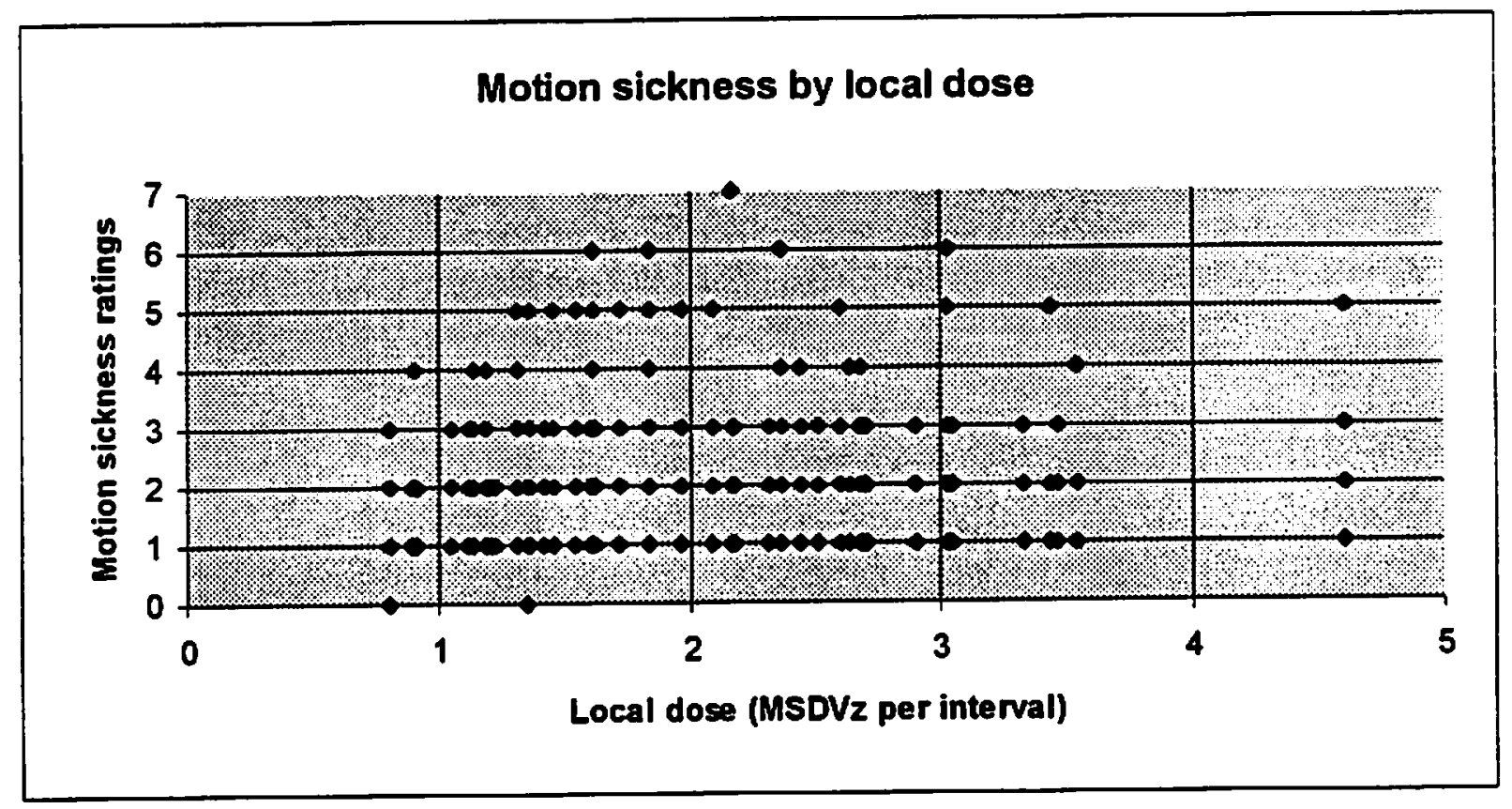

Figure 4-10. Scatter plot of motion sickness ratings for individual intervals.

\subsection{Discussion of Correlation between Duration and Subject Ratings}

Subjects' motion-sickness ratings did show significant correlations with one independent variable duration (as represented by "Interval" in their responses). As shown in the figure below, there is a slight, but significant downtrend in the number of subjects reporting they feel "perfectly normal", offset by substantial increases in those feeling "Slightly queasy" and smaller increases in those feeling worse. 


\section{Frequency of response levels by interval}

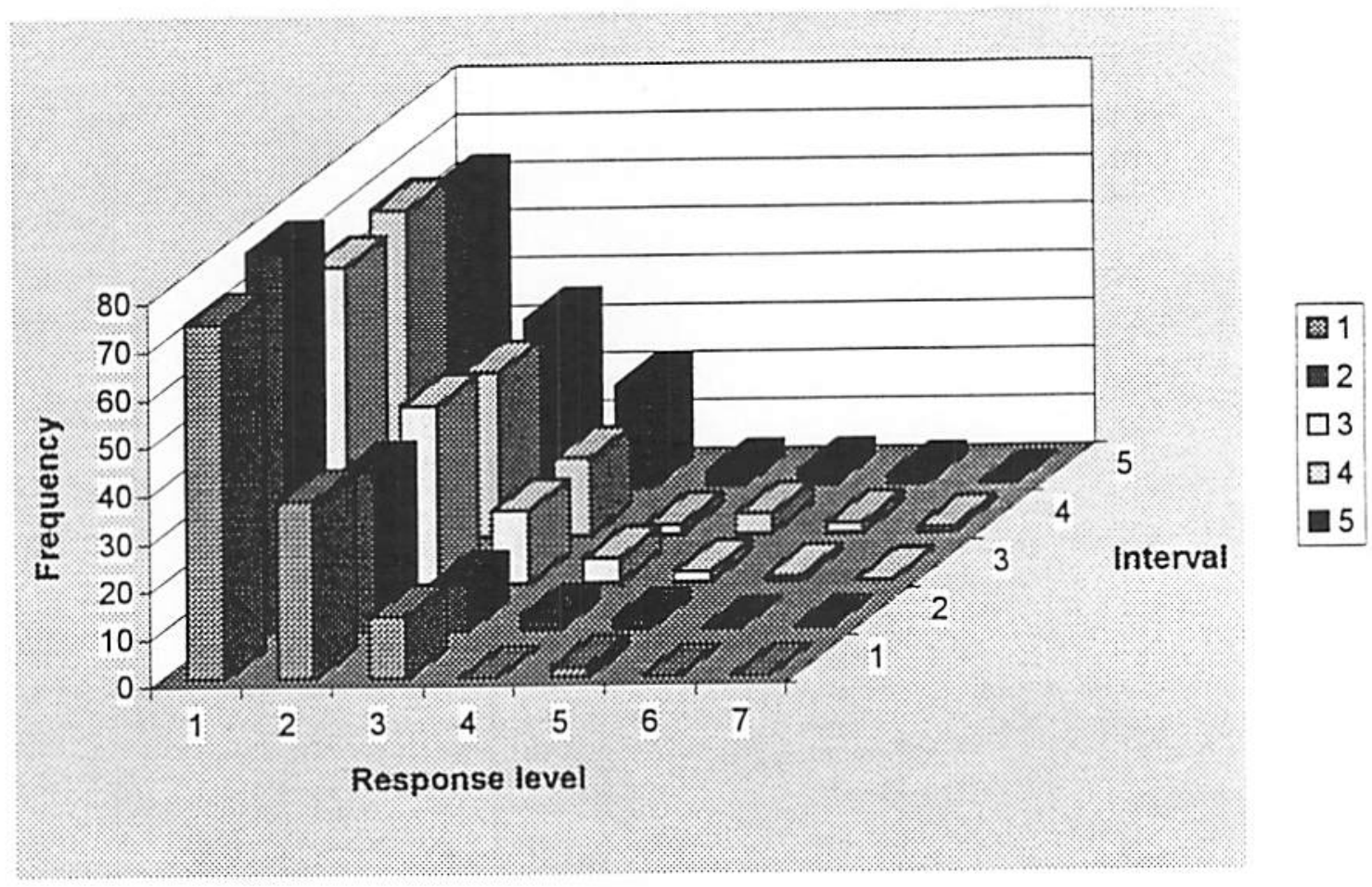

Figure 4-11. Motion sickness ratings by interval, summed across flights.

\subsection{Comparison of Flights with Simulations}

No one came close to vomiting in the simulator, while two subjects did so aboard the airplane. (The second instance of vomiting occurred just after the end of interval five and does not show up in the ratings data.) In fact, slightly more than half the subjects felt "perfectly normal" throughout the simulator trip, while only about $38 \%$ of the airplane subjects felt that well. 


\section{Maximum reported motion sickness for flights and simulators}

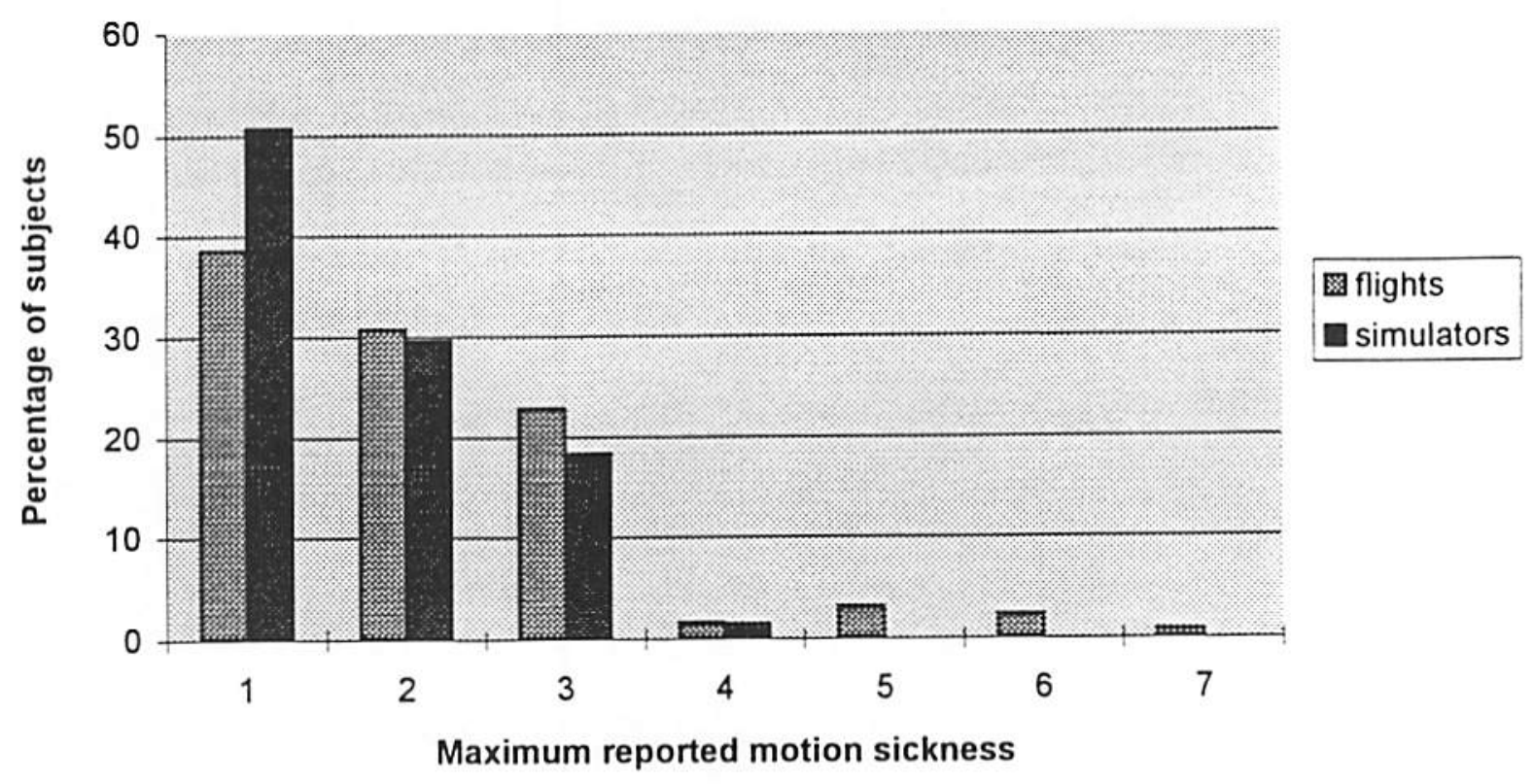

Figure 4-12. Comparison of motion sickness ratings between the airplane and the simulator.

However, subjects rated the ride comfort of the simulator as distinctly inferior to that of the airplane, as shown in the following figure. Fewer than a quarter of the subjects on the simulator found every interval to be "comfortable" or "very comfortable," while about $60 \%$ of the subjects on the airplane so reported. Nearly $40 \%$ of the subjects rated at least one portion of the simulator trip as "somewhat uncomfortable" or worse, while only about $15 \%$ of them did so while riding on the airplane. 


\section{Maximum ride-comfort ratings for flights and simulators}

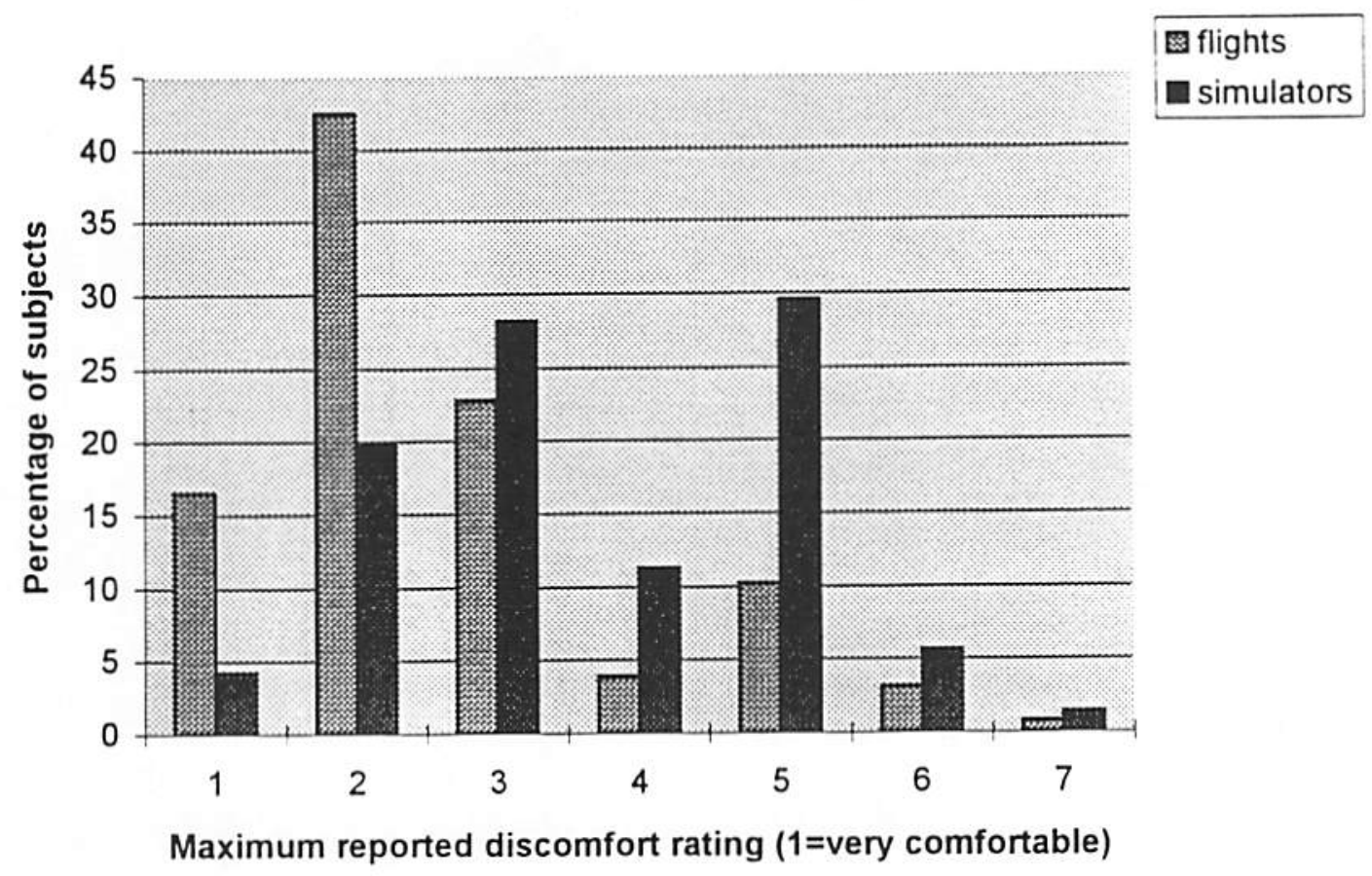

Figure 4-13. Comparison of ride-comfort ratings for flights vs. simulator trips. 
This disparity is most likely attributable to the annoying lateral forces experienced in the simulator, which are not present at all in flight, and not likely to occur in a Maglev or other high-speed ground system. Figure 4-14 shows a record of the roll rates and accelerations in all three axes experienced in the simulator during a 14-minute trip with simulated (i.e., visual) bank angles of $28^{\circ}$ and roll rates of up to $8 \%$ sec. Note that lateral acceleration $(\mathrm{Xg})$ hit peak values of about 30 centi-g on several occasions.

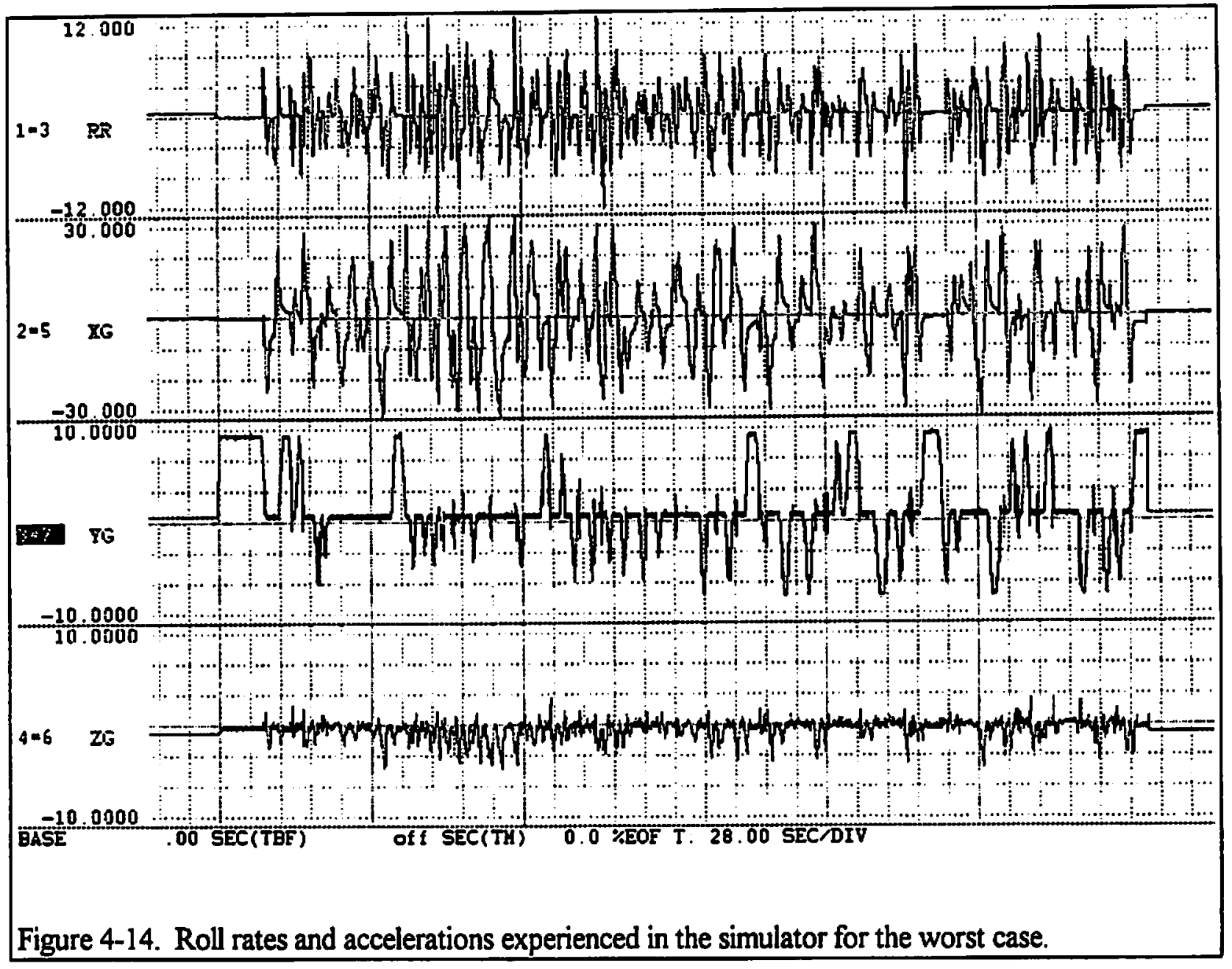




\section{Conclusions}

There were no significant statistical correlations between the subject ratings and the physical variables (vertical accelerations and roll rates) over the ranges tested. Nonetheless, several important conclusions can be drawn from this study:

1. The vast majority of subjects found the airplane simulation comfortable, even though in that simulation they experienced a motion environment considerably less comfortable than an actual Maglev or other high-speed ground system would produce. The average comfort rating for the plane trip ranged from 1.5 to 2.64 over the nine flights. These ratings were based on a seven-point scale where 1 is very comfortable, 2 is comfortable, 3 is somewhat comfortable, 4 is neutral, 5 is somewhat uncomfortable, 6 is uncomfortable and 7 is very uncomfortable. $82 \%$ of the 127 subjects rated every interval as somewhat comfortable or better on the airplane.

In the ground-based simulation, the average comfort rating varied from 1.68 to 4.57 over the 18 sessions, using the same rating scale described above. $52 \%$ of the 71 subjects rated every interval as "somewhat comfortable" or better.

2. Motion sickness was not a problem for the majority of subjects. On the flights, $69 \%$ never felt even slight queasiness at any point, while $23 \%$ felt slight queasiness, but nothing worse. Eight percent ( 10 out of 127) felt "intermittently nauseous" or worse at least one time in flight. Two subjects vomited during the flights. The Griffin model had predicted that for 127 subjects exposed to the dosages given, 1.92 would vomit. This very close correspondence between the model and actual results may have been a coincidence, but also suggests that the extension of this methodology to the evaluation of other modes such as Maglev is not unreasonable.

On the simulator, no one experienced definite nausea or vomiting and only one subject out of 71 reported intermittent nausea. $18 \%$ (13 subjects) reported slight queasiness, and more than $80 \%$ of the subjects were free of any symptoms of motion sickness. The relative lack of motion-sickness problems on the simulator was expected because it cannot produce sustained vertical accelerations, which are the major contributor to kinetosis.

3. Based on the results of this study there is no evidence that more than a small percentage of Maglev passengers would experience kinetosis on routes confined to the boundaries of existing highway rights of way. This study simulated a Maglev system traveling through representative portions of the proposed New York State route at average speeds that ranged from 320 to 400 $\mathrm{kph}(200$ to $250 \mathrm{mph})$. While the vertical accelerations experienced by the subjects in the aircraft simulation were generally greater than those that would be experienced by Maglev passengers only 2 of the 127 subjects vomited. 
4. At the start of this study, higher limits for maximum bank angle and roll rate were contemplated, based on previous work with isolated maneuvers. However, because of concerns that motion sickness might be far more prevalent at these higher limits when the rolling maneuvers were separated by only a few seconds, two pilot tests were conducted using about 30 personnel associated with various Maglev research projects supported by the U. S. Department of Transportation. Based on the reactions of these subjects, ride quality ratings would have declined sharply, while the incidence of motion sickness would have increased sharply, had the subjects been exposed to roll-rate limits of 10 or $12 \% \mathrm{sec}$ and bank angles as high as $40^{\circ}$. More than half of the participants on the pilot tests reported queasiness or worse under these higher limits. These pilot-test ratings were the basis for the decision to limit the exposure of the public subjects to $8^{\circ} / \mathrm{sec}$ in roll and $28^{\circ}$ in bank angle.

5. Among the independent variables in the experiments (maximum bank angles and roll rates, and duration of exposure), duration was the only significant predictor of subjects' motion sickness ratings. Flights were divided into five rating intervals, of eight to ten minutes each. Only two subjects felt "Intermittent nausea" in the first interval, while nine subjects were "Intermittently nauseous" or worse by the fourth interval. One subject vomited in the fourth interval and another just after the fifth. Had the experiments lasted longer, it is likely that some additional subjects would have reported motion-sickness symptoms.

6. The visual effects experienced in the ground-based simulator did not cause significant problems. Only one subject reported "intermittent nausea" in the simulator and none experienced any worse symptoms. Even though the windows were simulated with 35 " video monitors, the proportion of the total visual field filled with moving images was sufficiently small to avoid creating problems for passengers. Since actual Maglev vehicles are likely to have smaller windows than the simulator, there is no reason to expect that significant numbers of future Maglev passengers will be adversely affected by seeing the scenery rushing past.

7. On most flights, the actual vertical-acceleration dosage was significantly greater than the theoretical dosage that should have been accumulated by a Maglev traversing a guideway built to the nominal limits. This extra vertical acceleration arose from several sources including: (1) turbulence in the atmosphere; (2) altitude changes; (3) corrections of drift in the bank-angle measurement instrumentation; (4) extra turns required to keep the aircraft within the restricted air space; and (5) pilot error in following the displayed attitude indication. The peak roll rates were two to four degrees per second higher than intended on each flight. Hence the ratings developed here are conservative. An actual Maglev would not be subject to any of these sources of vertical acceleration, and would generate less vertical acceleration in the 0.1 to $1 \mathrm{~Hz}$ range than the plane flight.

8. Because subject comfort and motion-sickness ratings were essentially randomly distributed across the nine flights, it must be concluded that for a small fraction of the population (the $8 \%$ who felt more than slightly queasy in the study), even a very modest amount of rolling is

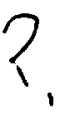


uncomfortable. Such persons will likely avoid Maglev, except on routes which are relatively straight and flat. For the remainder of the population, bank angles at the high end of the tested range are acceptable, even when roll maneuvers are occurring every 15 or 20 seconds.

9. Both the airplane and simulator experiments contributed to our understanding of ride-quality and motion-sickness issues in high-speed ground systems. Other questions, such as limits on longitudinal acceleration, were not addressed in this study, but will require examination in simulator tests prior to actual system design 


\section{References}

1. Sussman, E.D., Pollard, J.K., Mengert, P., DiSario, R. (1994) Study to Establish Ride-Comfort Criteria for High-Speed Magnetically Levitated Transportation Systems. U.S. Dept. of Transportation, Federal Railroad Administration, Washington, DC DOT/FRA/NMI-94/1.

2. Alexander, S.J., Cotzin, M., Klee, J.B., Wendt, G.R. (1947). Studies of motion sickness:XVI. The effects upon sickness rates of waves of various frequencies but identical acceleration. Journal of Experimental Psychology, 37, 440-447.

3. Griffin, M.J. (1991). Physical characteristics of stimuli provoking motion sickness. Paper 3 in: Motion Sickness: Significance in Aerospace Operations and Prophylaxis. AGARD Lecture series LS 175, ISBN 92-835-0634-0.

4. Guignard, J.C., McCauley, M.E. (1982). Motion sickness incidence induced by complex periodic waveforms. Aviation, Space and Environmental Medicine, 53, (6), 554-563.

5. Griffin, M. J. (1990). Handbook of Human Vibration, Academic Press, London.

6. International Organization for Standardization, (1994). Mechanical vibration and shock - Evaluation of human exposure to whole-body vibration - Part 1. Draft International Standard, ISO/DIS 2631-1.

7. Berger, Lehman Associates, P. C., (1992). Maglev Demonstration Project Site Location Study.

8. British Standard, (1987). Measurement and evaluation of human exposure to whole-body mechanical vibration and repeated shock. BS 6841: 


\section{Appendix A. COMBO.BAS: Overview and Explanation}

\section{Purpose}

The purpose of this appendix is to provide an overview and explanation of the computer program COMBO.BAS. The discussion begins by explaining what the program does and how to use it and then explains how the program works by describing the program flow and the kinematic formulas it uses. The annotated code appears in Appendix B.

\section{Overview}

COMBO.BAS is a QUICKBASIC program which calculates a minimum time "speed profile" from a 2 dimensional "Eurvature trajectory" (a curve in the $x-y$ plane). The term "turvature trajectory" refers to an idealized description of a physical guideway/track geometry. "Speed profile" refers to a sequence of velocities and bank angles.

The objective of COMBO.BAS is to calculate the fastest speed profile possible given the input trajectory and maximum values for velocity, bank angle, roll rate, acceleration and deceleration. Furthermore, the velocities, bank angles and curvatures are "balanced" in that all accelerations are resolved along the vertical axis. Thus, a passenger traversing the route according to the resulting speed profile would experience no lateral accelerations. Implications are that lateral acceleration constraints are automatically satisfied, since lateral acceleration is everywhere zero. Further implications are that there is no lateral jerk, as lateral acceleration is constant; thus lateral jerk limits are automatically satisfied. The final implication is that the speed is sometimes less than that which could have been allowed were balance not required.

Typically, one can expect travel time to decrease when the parameters (maximum velocity, bank angle, roll rate, acceleration, and deceleration) are increased. A valuable use of COMBO.BAS is to allow experimenting with these parameters to precisely determine their effect on travel time.

\section{Description of Input}

The program COMBO.BAS takes as input a geometric object - a curvature trajectory. COMBO.BAS does not in any way alter this input geometry. Based on this geometry COMBO.BAS calculates velocities and bank angles for traveling through this sequence of curves. Thus, it is assumed that an alignment (but not a guideway with fixed bank angles) has already been determined.

For input purposes the geometry (i.e. curvature trajectory) must be described by the radius of curvature (infinite for tangent sections) every delta $s$ (100). feet'. To use COMBO.BAS the user must provide the data in this form. However, it is possible to take data of a different form and convert it to this radius/distance form. For example, for this study the geometric description provided by the State of New York (NYRDY.DAT) consisted of a sequence of curves of varying lengths, along with a

\footnotetext{
'The unit of distance (delta s) must be small enough that kinematic changes over each segment are negligible.
} 
prescription for spirals. Thus, it was necessary to transform these data into the "curvature trajectory form" described above. The program (ALIGN.BAS) discussed in Appendix D accomplished this conversion. The output of ALIGN.BAS, a file called RECONST.ROE, was input to COMBO.BAS.

\section{Description of Output}

The primary output of COMBO.BAS is a "speed profile", that is, a sequence of velocities and bank angles. The output sequence also contains the following information: segment number, distance along route, radius of curvature, cumulative travel time, roll rate, and a reasons code. The reasons code documents the last constraint which caused a change in velocity for each segment. The sequence is provided in a "Constant distance" form (one record every delta-s feet) and in a "constant time" form (one record every delta-t seconds). These are saved in a "ARC" file and a "TIM" file respectively. Both forms are useful; in particular the "TIM" file is used to estimate a motion sickness dose value (as explained in Appendix C).

\section{Introduction to Program Architecture}

Given a flat planar alignment, i.e. a set of curves in the $x$-y plane, the primary focus of this effort is to determine a speed profile that traverses the curve in minimum time under constraints on:

1. speed

2. bank angle

3. acceleration

4. deceleration

5. roll rate

For the purpose of understanding the underlying program architecture it is helpful to distinguish between two types of constraints. Immutable constraints depend only on the point of computation along the curve whereas dymamic constraints require consideration of points before and after the computation point. Maximum allowable speed and maximum allowable bank angle are constraints that impose immutable restrictions. The speed must be kept below both (1) the maximum speed and (2) the speed implied by the local curvature and maximum bank angle. On the other hand, constraints such as acceleration, deceleration, and roll rate impose relative or dynamic limits, because as the vehicle moves from segment to segment the locally computed speed must accommodate speeds on segments that come before or after the computation point.

Recall that the input is a sequence of radii of curvature every 100 feet. From these radii, a sequence of speed limits is calculated based on balancing the accelerations and assuming the maximum bank angle (BANK). These speed limits are viewed as a sequence of posted speed restrictions every 100 feet. They are input to the primary loop as the initial values for the speed profile. The primary loop then determines whether any of the 5 above constraints are exceeded. Whenever an exceedance is obtained, the program reduces the vehicle speed. Also, whenever a current speed is found to be less than all applicable constraints, the program increases the speed. The process is repeated (iterated) several times, producing a final speed profile which minimizes transit time and adheres to the 5 constraints at all points. 
Description of COMBO.BAS: Program Flow Control and Algorithms

COMBO.BAS consists of a main module and various sub-modules. Figure Al presents a flow chart of the program.

\section{Main Module}

The main module takes inputs via a user interface for the limiting parameter values, file names, and several options. Next, the module performs set up operations (including declaring subroutines, setting flags for output options, and initializing variables). In addition, it provides tags which are used to record the speed limiting reasons at every point along the route. It then calls balance which calculates the speed limits (which serve as initial values for vehicle speed) and stores the result in ROLLX.DAT (a disk file). The bulk of the processing is done in a loop 'FOR ITERATION=1 TO IMT'. Upon completion of these iterations a solution is stored in ACTY.DAT. Then the main module calls several modules which perform three functions: 1) reparameterize to time, 2) calculate bank angle, 3) smooth the profile. The results are stored in the ".ARC" (one record every delta-s feet) and "TIM" (one record every delta-t seconds) files.

\section{Subroutine Balance}

Balance calculates the "speed limits"based on the local curvature (input file RECONST.ROE) and the maximum bank angle (input parameter BANK). The formula used for this calculation is the balanced lateral equation:

$$
\tan (\theta)=\frac{V^{2}}{g R}
$$

This formula relates the radius of curvature (R), the velocity $(V)$ and the bank angle $(\theta)$. It is used in other modules as well as in BALANCE; it is sometimes used to calculate a velocity (as in BALANCE where $\theta$ is taken to be the maximum allowable bank angle) and sometimes used to calculate a bank angle (for a given velocity).

In addition to calculating a speed based on the curvature and maximum bank angle, BALANCE checks that the speed implied by the balance equation does not exceed the user input maximum speed (LINESPEED) as may occur along straight segments or segments with a large radius of curvature (gentle curves). In such a case, the (immutable) "speed limit" computed by BALANCE is set equal to the LINESPEED. This process of checking speeds against the maximum is carried out by several of the modules. Thus there is no separate module for checking for speeds greater than LINESPEED. 
Figure Al. Flow Chart for Combo.bas

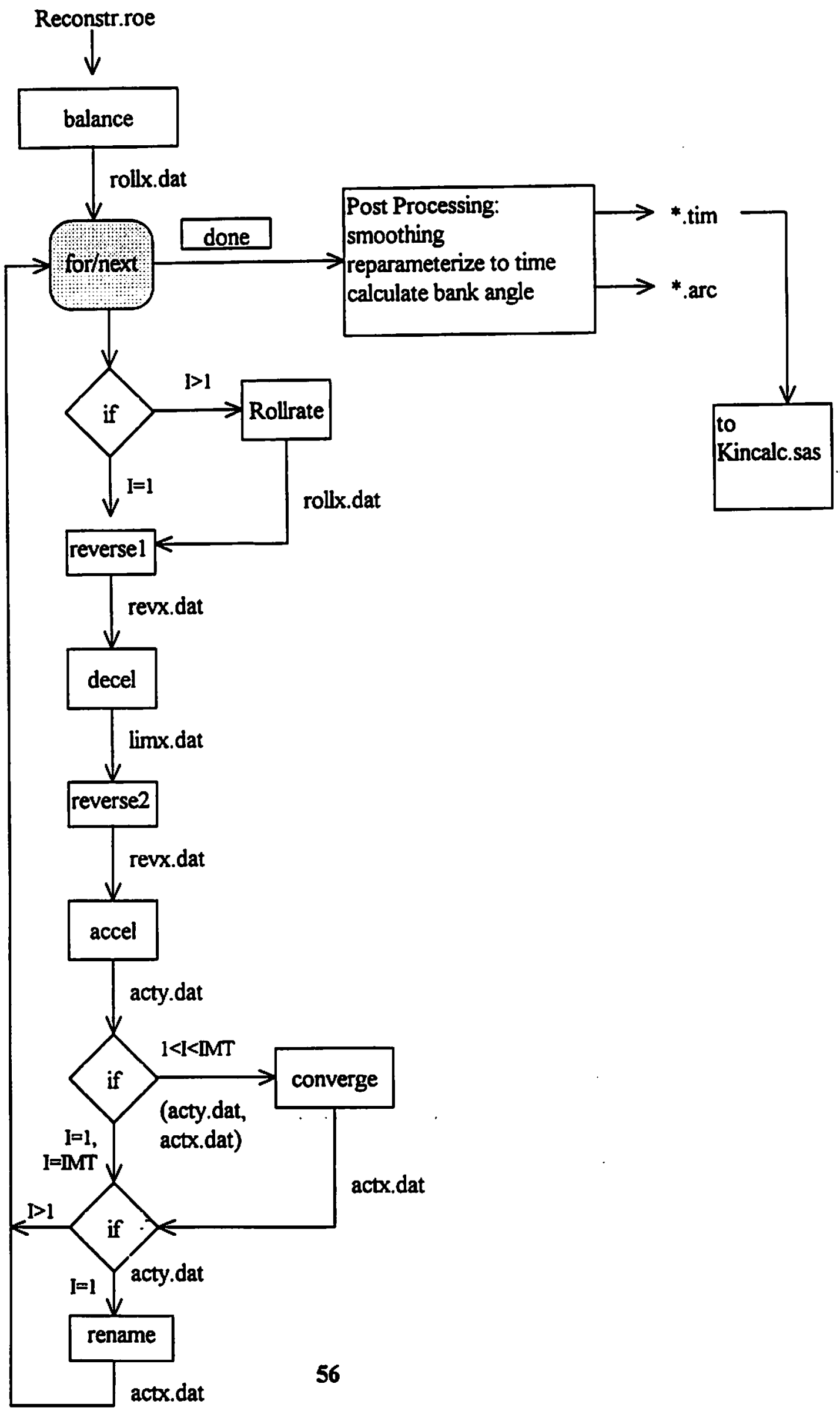




\section{Primary Loop}

The minimization of transit time is accomplished by a for/next loop in the main module. This loop calls several modules which impose the restrictions on roll rate, acceleration, and deceleration (ROLLRATE, ACCEL, DECEL). The loop also calls modules which perform clerical functions (REVERSE1, REVERSE2 and RENAME) and one module which aids in convergence (CONVERGE). At the completion of each iteration a new (updated) speed profile is output.

The steps in a typical iteration are as follows:

1. Determine the bank angles and roll rates based on current values for velocities. At each point along the curvature trajectory, limit velocity to meet the bank angle restriction or (if bank angle restriction does not apply) modify velocity (increase or decrease) to a value which makes roll rate closer to its given limit value.

2. Check for acceleration and deceleration limit violations. Where necessary, decrease the velocity to meet these restrictions.

3. Average the resulting speed profile with the speed profile obtained in the previous iteration. This is done to ensure convergence.

\section{Return.}

\section{Subroutines ACCEL and DECEL}

The purpose of the modules ACCEL and DECEL is to ensure that (longitudinal) acceleration and deceleration limits are obeyed. The code for subroutines ACCEL and DECEL is identical. ACCEL "looks behind" while going forward over the route from origin to destination. DECEL "looks behind" while going backward (which is effectively looking ahead) over the route from destination to origin. The reversal of time relationships (accomplished by modules REVERSE1 and REVERSE2) converts acceleration into deceleration and "look behind"into "look ahead". Combined, subroutines ACCEL and DECEL compute a speed profile which meets acceleration/deceleration limits at every point over the entire route.

The strategy which is used is "pedal-to-the-metal", whenever a change in speed is needed, the change is done using the maximum acceleration or the maximum deceleration. This method of "constant acceleration"is motivated by the goal of minimizing transit time. Simply put, there is never a reason for accelerating (or decelerating) at anything less than maximum value. Thus a well known formula from elementary kinematics is used: if a segment of length $s$ is covered at constant acceleration $a$ then

$$
V_{2}^{2}-V_{1 .}^{2}=2 \text { as }
$$

where $V_{2}$ is the speed at the end and $V_{1}$ is the speed at the beginning of the segment.

The module ACCEL has the surprising property that with a single pass through the data the acceleration limit is obeyed along the entire route. This is accomplished by always making sure the output speed is no greater than that which can be reached from the output speed for the previous 
segment under maximum acceleration (using the above formula). Through this one-step-at-a-time process, a severe speed reduction at one point can be felt at a consderable distance "downstream". Of course, DECEL has the analogous property: a single pass through the data ensures that the deceleration limit is never exceeded.

If acceleration, deceleration, bank angle and maximum speed were the only restrictions to be placed on the speed profile a single pass through the data (a single iteration) would suffice. Using the speed limits determined by BALANCE the program would need only reduce the speeds in the profile to the limits implied by ACCEL and DECEL. However, the roll rate restriction complicates this matter. Consider the following example. Suppose a candidate speed profile exceeds the roll rate restriction at segment $i+1$. The bank angle from segment $i$ to segment $i+2$ changes too rapidly. The speed is reduced to accomodate the roll rate restriction. As a result, the bank angle (implied by the new speeds and the balanced lateral condition) is also reduced from segment $i$ to segment $i+2$ (i.e., slower speeds through a fixed curve lead to gentler bank angles). This further reduces the roll rate at segment $i+1$. Also, this change affects roll rates at segments $i$ and $i+2$. Thus the consideration of roll rate constraints necessitates the iterative procedure.

\section{Subroutine ROLLRATE}

The module ROLLRATE introduces the roll rate restriction. It computes the rate of change of the bank angle and adjusts the velocity. By iteratively executing the roll rate module the program arrives at a velocity profile which meets the roll rate restriction. (Unlike the ACCELDECEL modules, the ROLLRATE module does not output a velocity profile which meets the relevant restriction in a single pass. It functions by replacing a velocity profile with one in which rolls are executed at rates that are closer to the limit value.)

The formula used by ROLLRATE for calculating the roll rate is based on the time derivative of bank angle:

$$
\left|\frac{d \theta}{d t}\right|=\left|\frac{d \theta}{d s}\right|\left|\frac{d s}{d t}\right| \approx\left|\frac{\Delta \theta}{\Delta s}\right| V
$$

where $\frac{\Delta \theta}{\Delta s}$ is the change in actual bank angle over a very short distance divided by that distance. If $\alpha$ is the maximum allowable rollrate, one could use

$$
V=\frac{\alpha}{\Delta \theta / \Delta s}
$$

to compute the velocity which meets the roll rate restriction. However, to avoid oscillation and ensure convergence, a geometric mean between the previous value (velocity from previous iteration) and the velocity implied by the above equation is calculated:

$$
V=\left(\frac{\alpha}{\Delta \theta / \Delta s} V_{\text {provious }}\right)^{1 / 2}
$$

The module ROLLRATE also checks to see that the bank angle restriction is met using the balanced lateral equation. 


\section{Subroutine Converge}

At the end of each iteration (except the first and last) the speed profile obtained is averaged with the speed profile obtained in the previous iteration. This step is included to aid convergence. Convergence was found to be sure and rapid on the data used.

\section{Subroutines for Final Output}

Having obtained the speed profile, the program performs 3 additional functions: 1 . calculate bank angle, 2. reparameterize to time, 3. smoothing.

\section{Subroutine ComputeConvergedBankAngle}

The subroutine ComputeConvergedBankAngle computes bank angle as a function of arclength given the speed profile. The inputs are curvature, speed squared, cumulative distance segment number and speed limiting reasons tag. The bank angle is calculated using the balanced lateral equation. The outputs are speed (not squared), bank angle, curvature, cumulative distance, cumulative travel time, rollrate, segment number and speed limiting reason tag every 100 feet. The output file name is user specified with a standard file name extension of "ARC".

\section{Subroutine ReparameterizeToTime}

Reparameterization is accomplished by REPARAMETERIZETOTIME, a module which outputs the velocity profile in equal time increments. The input file (*.ARC) contains speed, bank angle, curvature, cumulative distance, cumulative travel time, rollrate, segment number and speed limiting reason tag every 100 feet. The module linearly interpolates each of these values to obtain a value every 0.1 seconds. Other methods of interpolation could be used. Output file name is user specified with standard file name extension ".TM".

\section{Subroutines ForwardSmooth, BackwardSmooth and Average}

Exponential smoothing is performed in the forward direction (FORWARDSMOOTH) and in the backward direction (BACKWARDSMOOTH) and the results are averaged (AVERAGE). SMOOTHREVERSE1 and SMOOTHREVERSE2 are called to reverse the data order between forward smoothing and backward smoothing and after backward smoothing to restore the original order. 


\section{Appendix B. COMBO.BAS Annotated Code}

\section{Subroutine Balance Logic}

The Balanced Speed Section uses the alignment data as input and a user supplied parameter, the bank angle limit, to compute balanced velocity squared.

Input is a disk file (RECONST.ROE) containing curvature, cumulative distance and the segment number. Input parameters are bank angle limit and line speed limit.

Step 1) Bank angle limit is in degrees. It is converted to radians for computational uses.

Step 2) The balanced speed squared is computed using the maximum allowed bank angle and curvature for each segment piece.

Step 3) Computed speed squared is less than or equal to line speed limit squared.

Step 4) Outputs are the curvature, cumulative distance, balance speed squared, segment number and speed limiting reasons tag for each standard unit distance (100 feet). Output file is ROLLX.DAT.

\section{Program Logic: Deceleration}

Step 1) Convert deceleration limit in g's to deceleration limit in feet/second ${ }^{2}$.

Step 2) Check that prior tempVVS (speed squared from previous piece) is within line speed limit squared.

Step 3) Compute new temporary squared velocity using the constant deceleration formula: VVS = prior tempVVS + 2*Deceleration*distance.

Step 4) Compare the input speed squared value for the current segment to the (incremented) speed squared value from the preceding segment and use the smaller value.

Step 5) If the speed was changed by the Deceleration Subroutine adjust the speed limiting reasons tag.

Step 6) Output the results of considering deceleration as a limiting factor for the present piece.

\section{PROGRAM LOGIC: Acceleration}

Step 1) Convert acceleration limit in $\mathrm{g}^{\prime}$ s to acceleration limit in feet/second ${ }^{2}$.

Step 2) Check that prior tempVVS (speed squared from previous piece) is within line speed limit squared.

Step 3) Compute new temporary squared velocity using the constant acceleration formula: VVS = prior tempVVS + 2*acceleration*distance.

Step 4) Compare the input speed squared value for the current segment to the incremented speed squared value for the preceding segment and use the smaller value.

Step 5) If the speed was changed by the Acceleration Subroutine adjust the speed limiting reasons tag.

Step 6) Output the results of considering acceleration as a limiting factor for the present piece. 


\section{Program Logic: RollRate}

Step 1) Use the balanced lateral equation to compute the required bank angle based on the incoming speed profile for three pieces, the present piece ( $\# 2)$ and it's predecessor (\#1) and successor (\#3).

Step 2) Compute the rate of change of the bank angle with respect to distance by taking the central difference, the difference between bank angle \#3 less bank angle \#1.

Step 3) Compute an upper limit for speed squared for the present piece (\#2) using the lateral balance equation and the maximum allowed bank angle.

Step 4) Roll rate limited speed is computed as the rate of change of bank angle with respect to time ( $d$ theta/dt) divided by the rate of change of bank angle with respect to distance ( $d$ theta/dx) roll rate limited speed $=(d$ theta/dt $) /(d$ theta/dx $)=d x / d t$

Step 5) If $d$ theta/dx is not zero then compute the geometric mean of roll rate limited speed squared and previously computed speed squared by taking the square root of (roll rate limited speed squared times input speed squared)

Step 6) Compare the speed squared just computed to lateral balance speed squared computed in step 3 and retain the smaller.

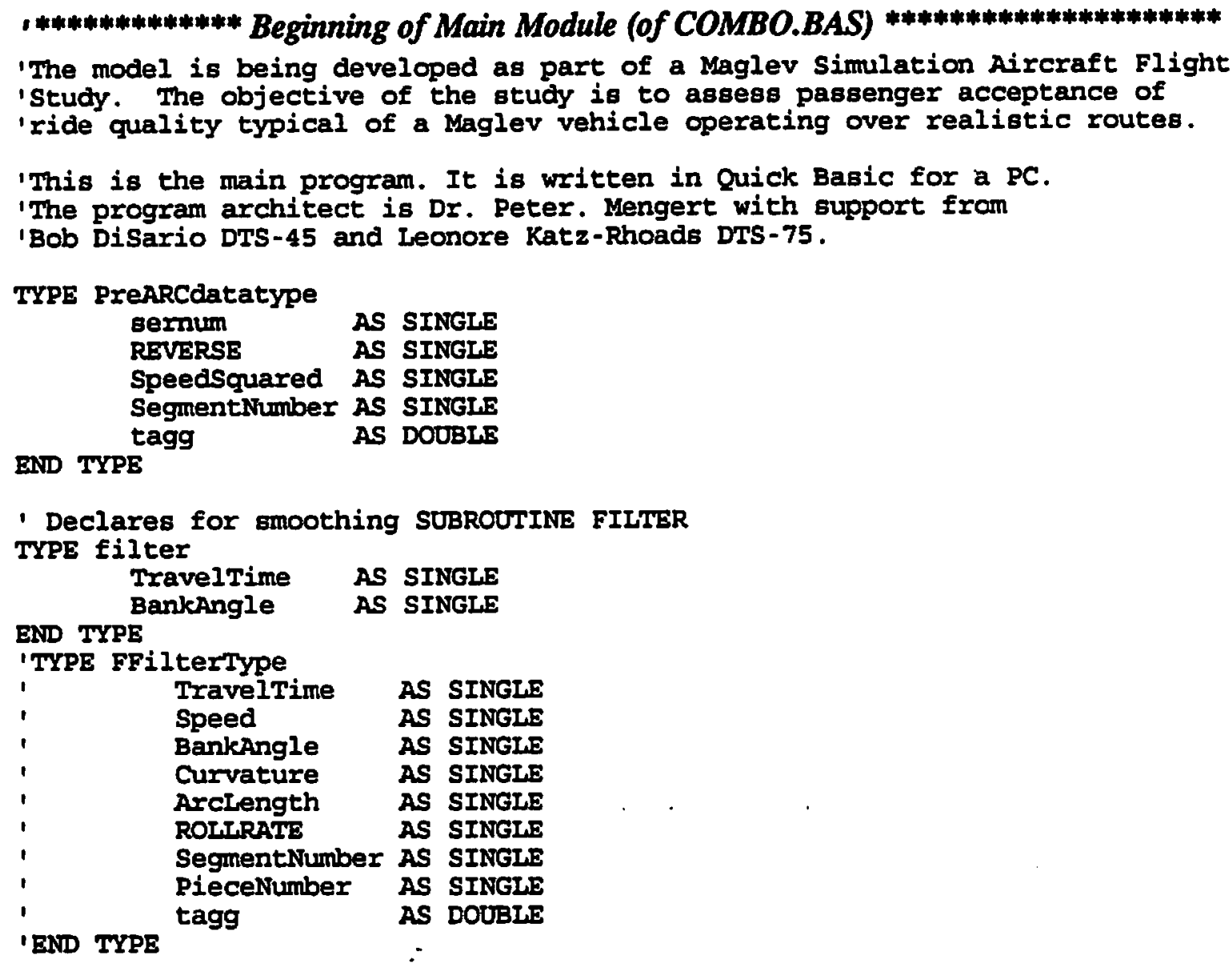


'tag tells where and when the program set speed values

' tag encodes 1) iteration: $I$, 2) module: $M$ 3) parameter: Pp

1 in the form "IMPP. Otherstuff"

1 where letter "I" tags when a speed value changed

1 the letter "M" tags where the speed value changed

- if $M=1$ then a speed value was set by ROLLRATE constraints

- if the tag is negative, rollrate increased speed

- if $M=2$ then a speed value was set by Deceleration

- if $M=3$ then a speed value was set by Acceleration

- PP is the parameter, roll rate in degrees/second

- or accel or decel in rage of $1 \mathbf{G}$

- the . conv data comes from the Converge subroutine

1 this subroutine combines two speed values. Therefore tags are

- also combined using this method;

- the New tag has the value IMPP. OTHERSTUFF

- the old tag has the value impp. otherstuff

- the combined tag: IMPP.imppoTHERSTUFF (otherstuff is ignored)

- Converge only combines if the new and old tag differ by $1.0 \mathrm{e}-8$ or more

- if tags are not that different Converge just passes the new tag along

DECLARE SUB BATANCE (infiles, BANK, LineSpeed, tagg\#) '

DECLARE SUB ROLLRATE (MaxRolirate, BANK, LineSpeed, UpHandle, tagg\#, IogFile\$)

DECLARE SUB REVERSE1 ()

DECLARE SUB Deceleration (ACCEL, IineSpeed, tagg\#, LogFile\$)

DECLARE SUB REVERSE2 ()

DECLARE SUB Acceleration (decel, Inespeed, tagg\#, LogFile\$)

DECLARE SUB Converge (Logfiles)

DECLARE SUB ComputeConvergedBankangle (INDAT\$, OUTDAT\$, style, IogFile\$)

DECIARE SUB ReparameterizeTOTime (INDAT\$, OUTDAT\$, LOgFile\$)

DECLARE SUB ForwardSmooth (A1, InputFiles)

DECIARE SUB SmoothReversel (InputFiles)

DECLARE SUB BackwardSmooth (A1)

DECLARE SUB SmoothReverse2 ()

DECLARE SUB Average (JKDAT\$, LogFiles)

DECLARE SUB Fullsmoothaverage (InputFiles, JKDATS, LogFile\$)

1 The following SUBroutines are NOT called directly in the main program. DECLARE SUB REVERSE (infiles, outfiles)

DECLARE SUB SmoothReverse (infiles, outfile\$)

- AGAIN: is a label, it is the target of a GOTO at the end of the main program

- the user is given the option of running another case (Do it again?)

AGAIN:

IUSER INPUT SECTION PRINTS MESSAGES TO SCREEN AND ACCEPTS DATA

'Sign on banner. What are we? what version?

banner $\$$ "COMBO Version $9-6 / 12 / 95$ "

PRINT banner\$

PRINT "DATA IN [BRACKET] IS DEFAULT VALUE"

PRINT "Enter name and path of input file [RECONST.ROE=DEEAULT]"

INPUT InputFileNames

infile\$ = RTRIMS (InputFileName\$)

InputFileName\$ $=$ ITRIMS (infiles)

IF InputFileNames $="$ " OR InputFileName\$ = " THEN ImputFileName\$ = "RECONST. ROE"

PRINT "INPUT ROLIRATE [8]", BANK ANGLE [20], ACCEL [.04], DECEL, LINE SPEED [440] 


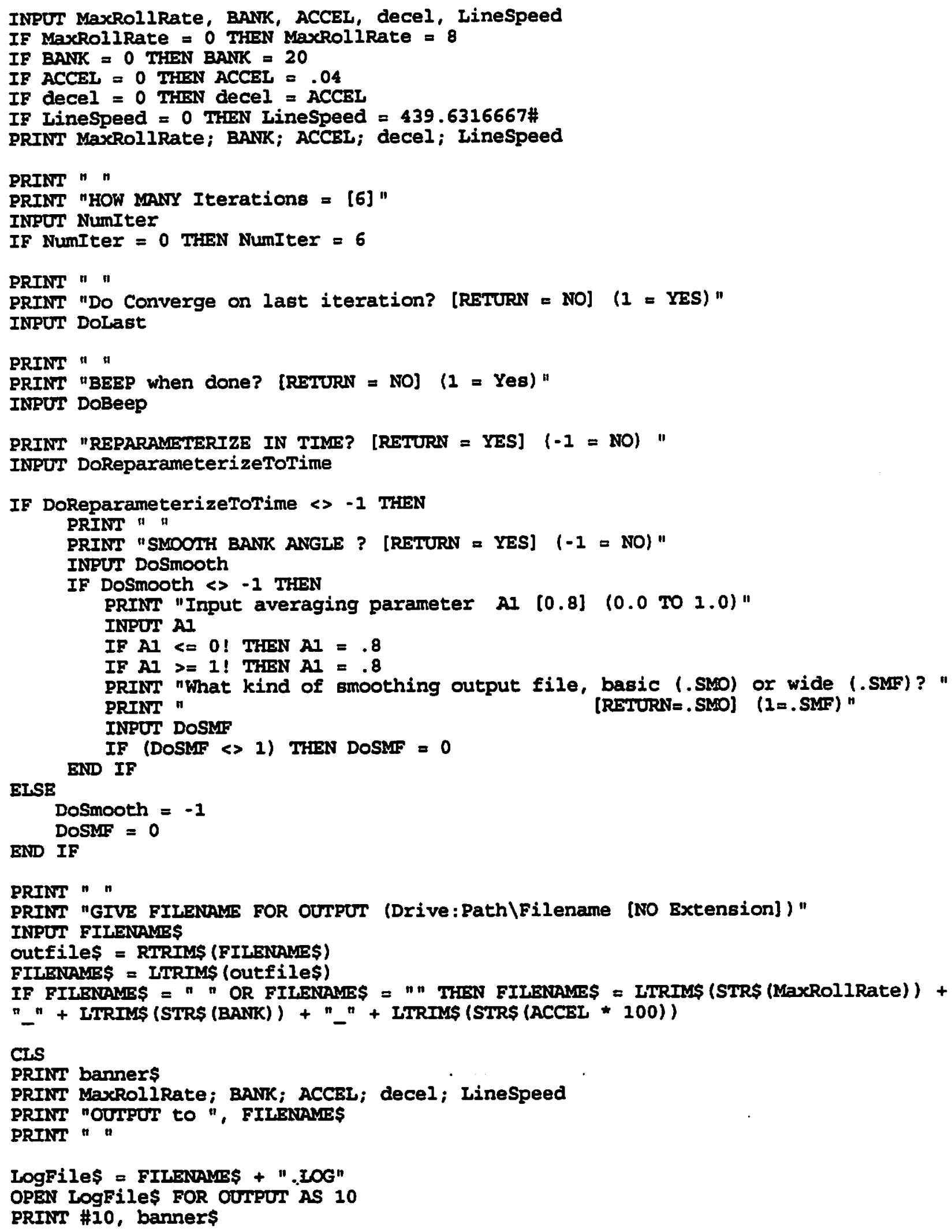




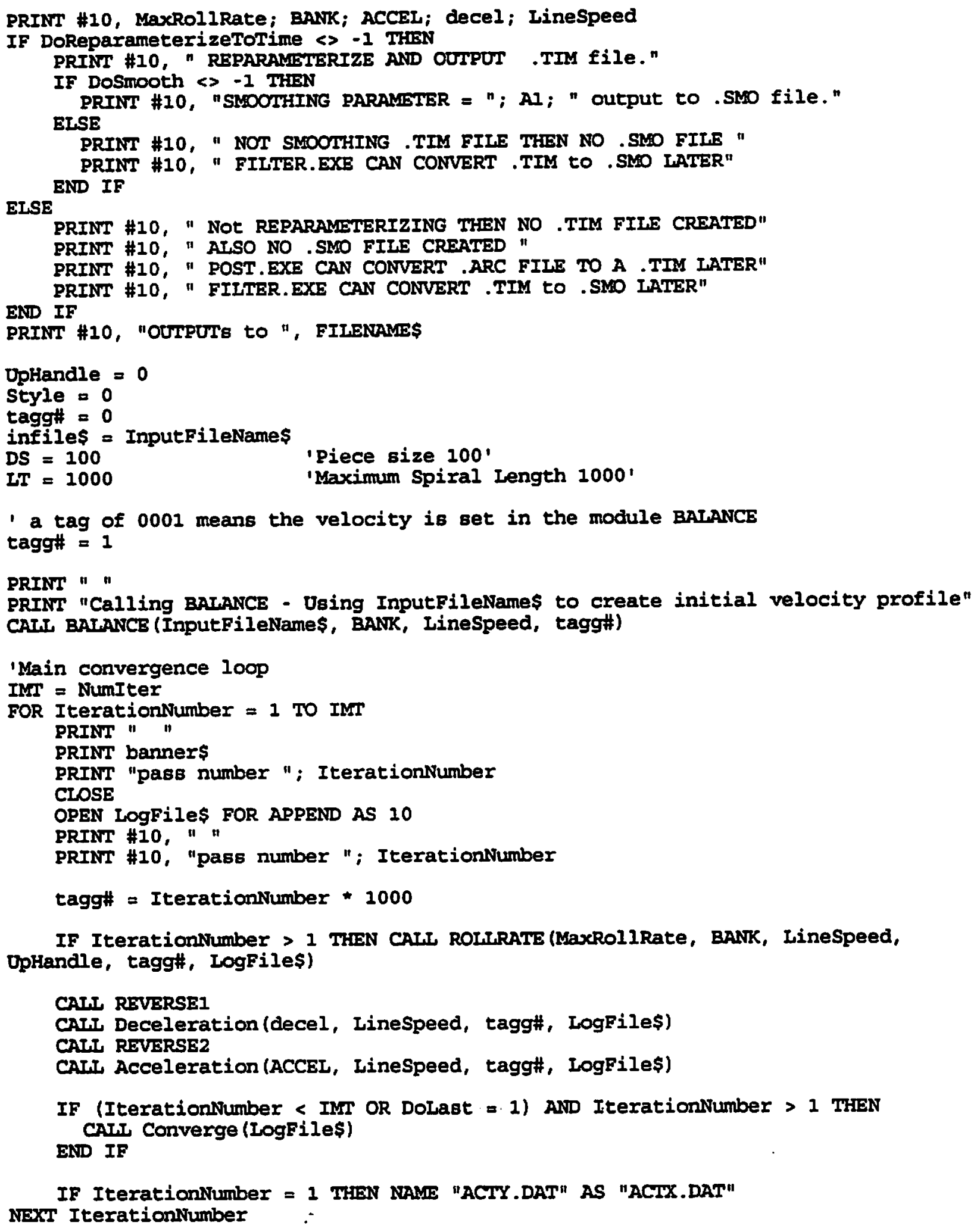




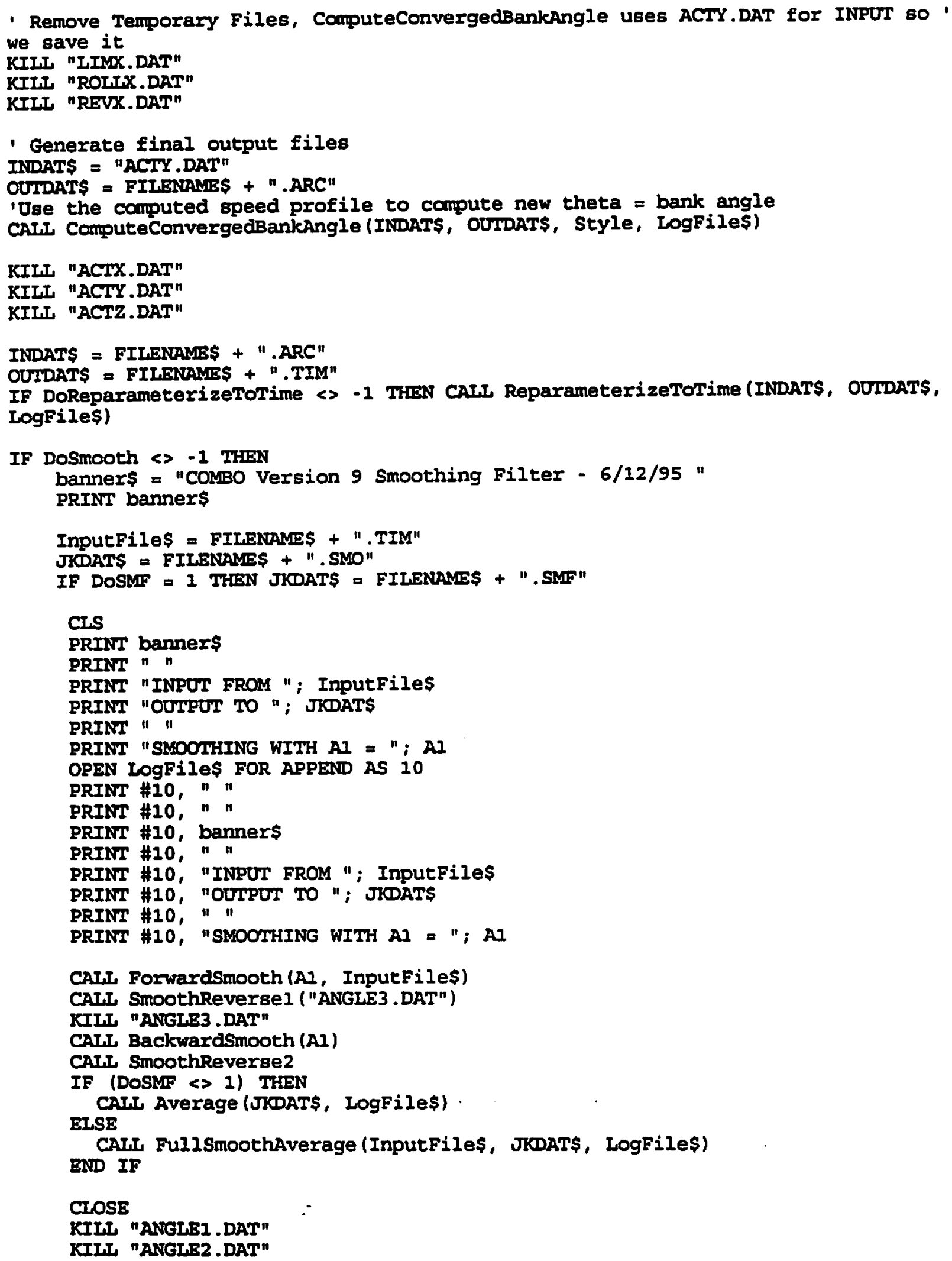


KIIJ "ANGIEREV.DAT"

KILL "FLIPPED.DAT"

END IF

IF (DOBeep $=1$ ) THEN BEEP

PRINT "Do another? ( $1=\mathrm{Yes}$, else=No)"

INPUT DOAnother

IF DOAnOther $=1$ THEN GOTO AGAIN

END

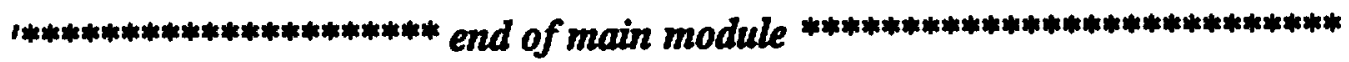

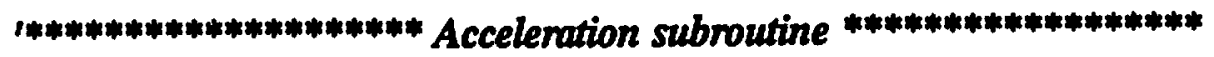

SUB Acceleration (ACCEL, Linespeed, tagg\#, IogFiles)

PRINT "Entered Acceleration"

$\mathbf{G}=32.2 \quad$ 'GRAVITY

$A=A C C E L$ * $G$ 'ACCELERATION AND DECELERATION "Step 1

vamax = IineSpeed * IineSpeed

um $=$ vamax

OPEN "REVX.DAT" FOR INPUT AS 1

OPEN "ACTY.DAT" EOR OUTPUT AS 2

tag\# = INT (tagg\#) $+300+\operatorname{INT}($ (ACCEL +.00001$) * 100 !)$

Numpieces $=0$

NumTouched $=0$

DO WHILE NOT EOF (1)

INPUT \#1, RQ, DSCUM, VVS, SegmentNumber, tagold\#

NumPieces $=$ NumPieces +1

IF un > vemax THEN Um = v8max

- Step 2

- Step 3

um $=u m+2 * A * 100^{\prime}$ CONSTANT ACCELERATION AS A FUNCTION OF DS

'VELOCITY SQUARED = VVS initial + 2as

'Newtonian Mechanics by A.P. French

IF VVS < Um THEN

- Step 4

un $=$ vvs

tagout\# = tagold\#

ELSE

tagout\# = tag\#

- Step 5

NumTouched $=$ NumTouched +1

END IF

ug $=u m$

IOOP

PRINT \#2, RQ; DSCOM; ug; SegmentNumber; tagout\# - Step 6

CLOSE

PRINT "Acceleration changed "; NumTouched; " of "; Numpieces

OPEN IOgFile\$ FOR APPEND AS 10

PRINT \#10, "Acceleration changed "; NumTouched; " of "; NumPieces 


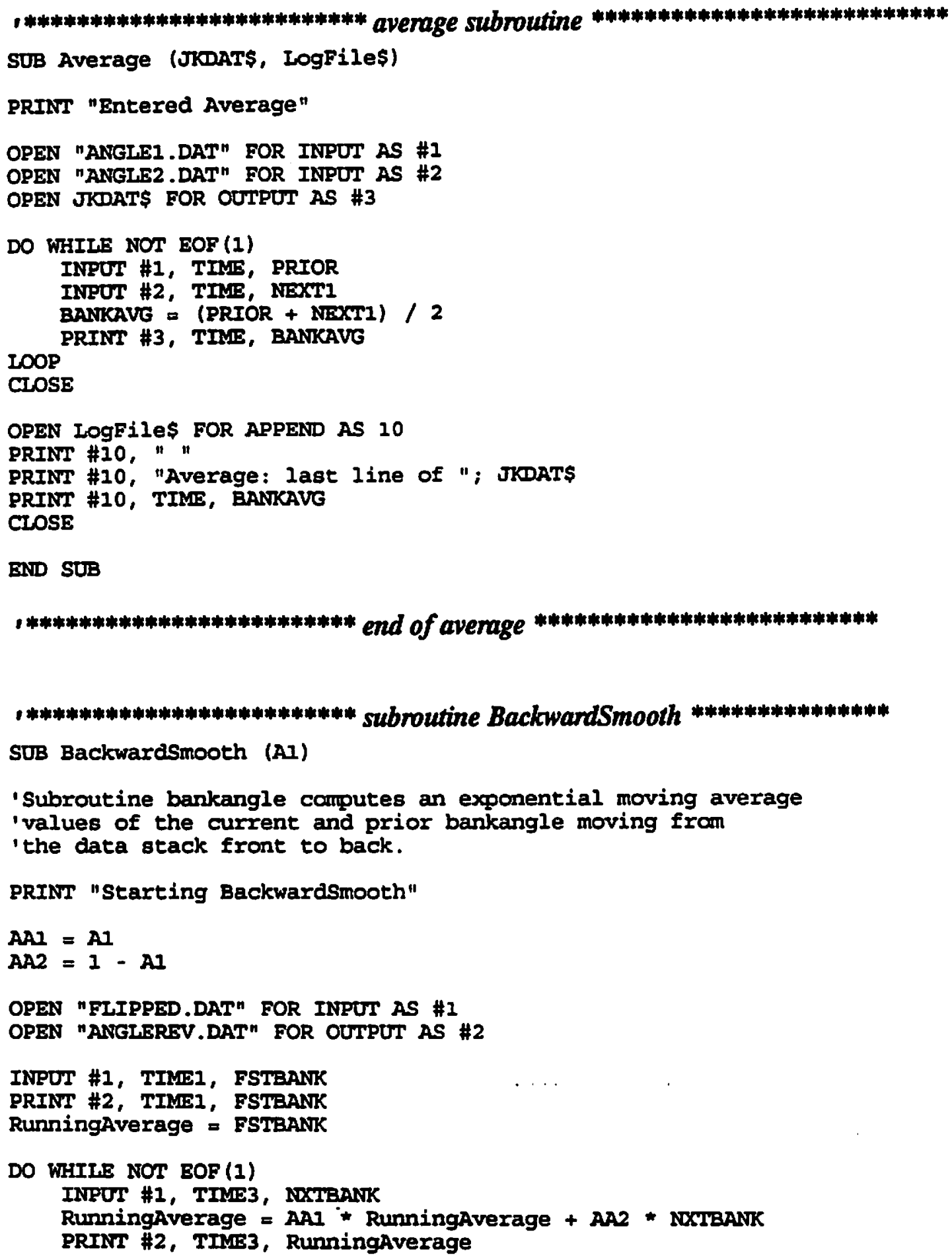

' Subroutine bankangle canputes an exponential moving average 'values of the current and prior bankangle moving fram 
END SUR

SUB BALANCE (infiles, BANK, LineSpeed, tagg\#)

$\mathbf{J}=0$

$G=32.2$

THETA $=$ BANK $\star$ ATN (1) $/ 45$

VVS $=0$

vsmax $=$ LineSpeed $*$ LineSpeed

tag\# = tagg\#

- TAG\# = tagg\# * bank

OPEN infileS FOR INPUT AS 1

OPEN "ROLIX.DAT" FOR OUTPUT AS 2

DO WHIIE NOT EOF (1)

INPUT \#1, CURVE, CUMFEET, SegmentNumber

IF CURVE $=0$ THEN

VVS $=999999$

ELSE

VVS $=$ TAN (THETA) *G / ABS (CURVE) $\quad$-Step 2

END IF

IF VVS > VBmax THEN VVS = vemax 'Step 3

LOOP

PRINT \#2, CURVE; CUMFEET; VVS; SegmentNumber; tag\#'Step 4

CLOSE

END SUB

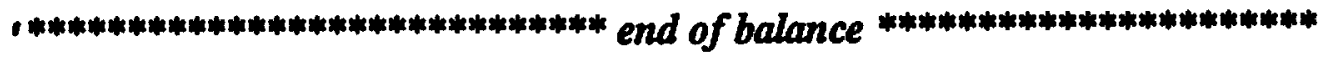

1**************** subroutine ComputeConvergedBankAngle *************

SUB ComputeConvergedBankAngle (INDAT\$, OUTDAT\$, Style, LogFile\$)

PRINT "Entered ComputeConvergedBankAngle, writing to "; OUTDAT\$

PRINT " reading from "; INDAT\$

OPEN INDATS FOR INPUT AS \#1

OPEN OUTDATS FOR OUTPUT AS \#2

$\mathbf{G}=32.2$

$\mathrm{dt}=.00001$

CumulativeTravelTime $=0$

'New Style - Starts with zero distance and zero bank angle, outputs first

- segment

PriorCumulativeDistance $=0$

PriorBankAngleDegrees $=0$ 
DO WHILE NOT EOF (1)

INPUT \#1, Curvature, CumulativeDistance, VS, SegmentNumber, tag\# $V=S Q R$ (VVS)

DD = CumulativeDistance - PriorCumulativeDistance

$\mathrm{dt}=\mathrm{DD} / \mathrm{v}$

CumulativeTravelTime = CumulativeTravelTime + dt

THETA = ATN (VVS * Curvature / G)

BankAngleDegrees = THETA $* 45 /$ ATN (1)

IF BankangleDegrees - PriorBankangleDegrees a 0 THEN RollRacevalue $=0$

ELSE

RollRatevalue = (BankAngleDegrees - PriorBankAngleDegrees) $/$ dt

END IF

PRINT \#2, V, BankangleDegrees, Curvature, Cumulativedistance,

CumulativeTravelTime, RoliRatevalue, Segmenthumber, tag\#

PriorCumulativeDistance = CumulativeDistance

IOOP

PriorBankangleDegrees = BankAngleDegrees

CLOSE

OPEN IOgFiles FOR APPEND AS 10

BRINT \#10, " "

PRINT \#10, "ComputeConvergedBankAngle: last line of "; OUTDAT\$

PRINT \#10, "Speed", "Bank Angle", "Curvature", "Distance", "TravelTime",

"Rolirate", " SegmentNumber", "ReasonCode"

PRINT \#10, V, BankAngleDegrees, Curvature, CumulativeDistance, CumulativeTravelTime, RollRateValue, SegmentNumber, tag\# CIOSE

END SUB

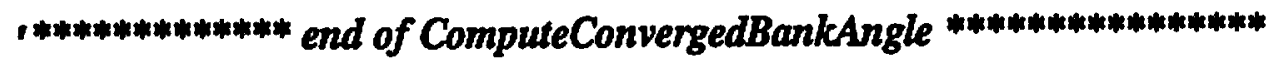

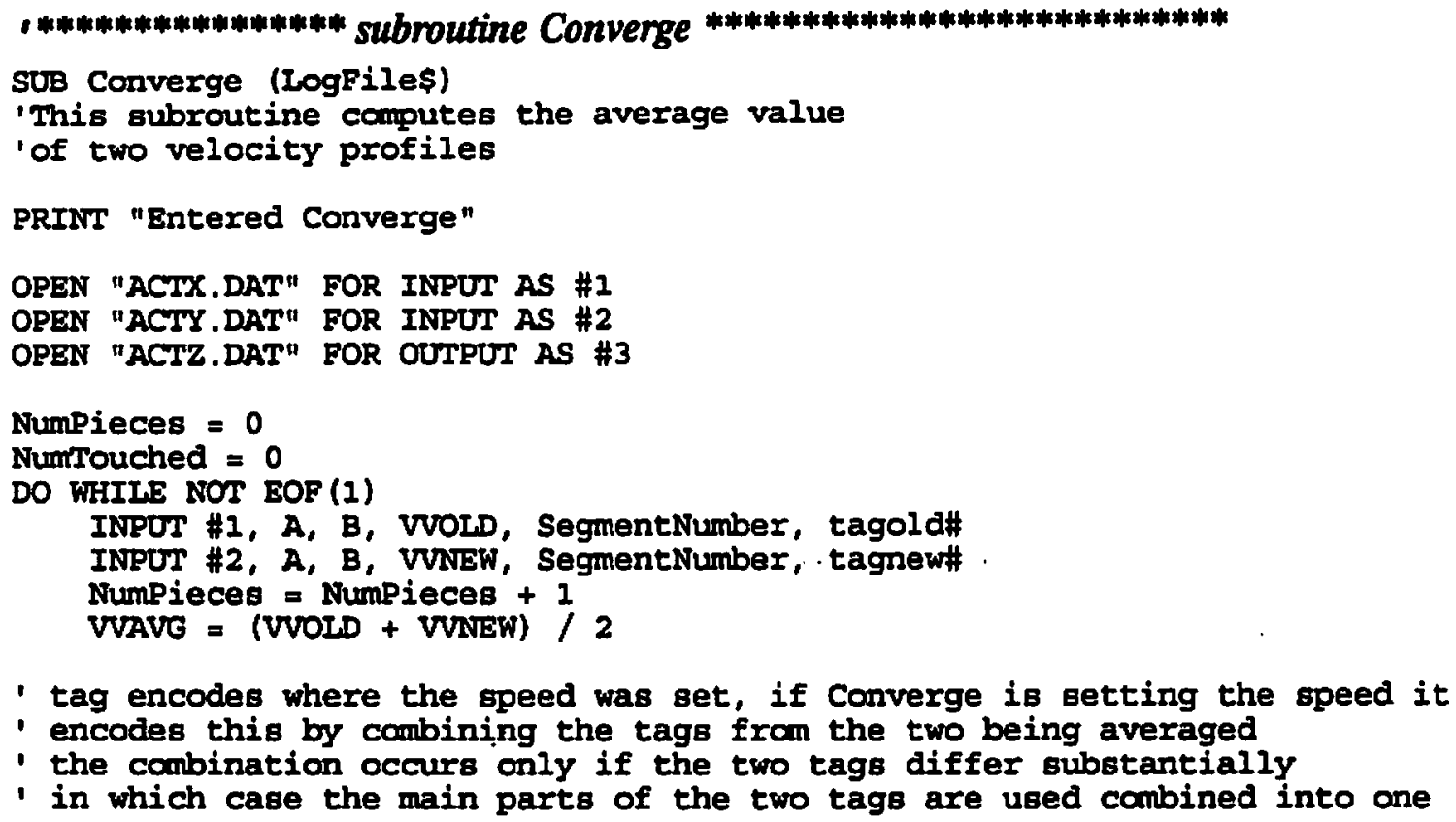


- value

- with the newer (i.e., later in the runsing) tag in the primary position

IF (ABS (ABS (tagold\#) - ABS (tagnew\#)) < 1E-08) THEN

'if the tags are essentially identical, just pass tagnew\# through tagout\# = tagnew\# ELSE

- take the OrD tag's main part (integer) and the NEW tag's secondary

- (fractional)

tagout\# = INT (ABS (tagold\#)) + ABS (tagnew\# - INT (tagnew\#))

1 the tag we output is the main part of the new, with the above combo as

- secondary

tagout\# = INI (ABS (tagnew\#) + (tagout\# / 10000\#)

NumTouched $=$ NumTouched +1

END IF

IOOP

PRINT \#3, A; B; VAVG; SegmentNumber; tagout\#

CIOSE

PRINT "Converge changed "; NumTouched; " of "; Mumpieces

OPEN IOgFile\$ FOR APPEND AS 10

PRINT \#10, "Converge changed "; NumTouched; " of "; Numpieces

OPEN "ACTZ.DAT" FOR INPUT AS \#1

OPEN "ACTX.DAT" FOR OUTPUT AS \#2

DO WHIIE NOT EOF (1)

INPUT \#1, A, B, C, SegmentNumber, tagold\#

IOOP

PRINI \#2, A; B; C; SegmentNumber; tagold\#

CLOSE

END SUB

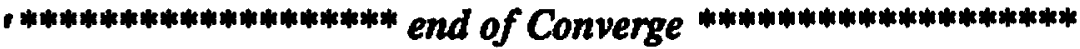

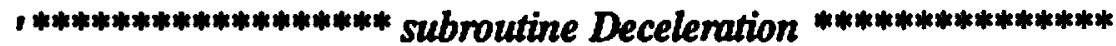

SUB Deceleration (decel, IineSpeed, tagg\#, IogFile\$)

PRINT "Entered Deceleration"

$G=32.2 \quad$ GRAVITY

$D=$ decel $* G \quad$ 'ACCELERATION DECELERATION 'Step 1

vomax $=$ LineSpeed * LineSpeed

um $=$ vemax

tag\# $=\operatorname{INT}($ tagg\# $)+200+\operatorname{INT}(($ decel +.00001$) * 100 !)$

OPEN "REVX.DAT" FOR INPUT AS 1

OPEN "LIMX.DAT" FOR OUTPUT AS 2

Numpieces $=0$

NumTouched $=0$

DO WHILE NOT EOF (1)

INPUT \#1, RQ, DSCOM, VVS, SegmentNumber, tagold\# 


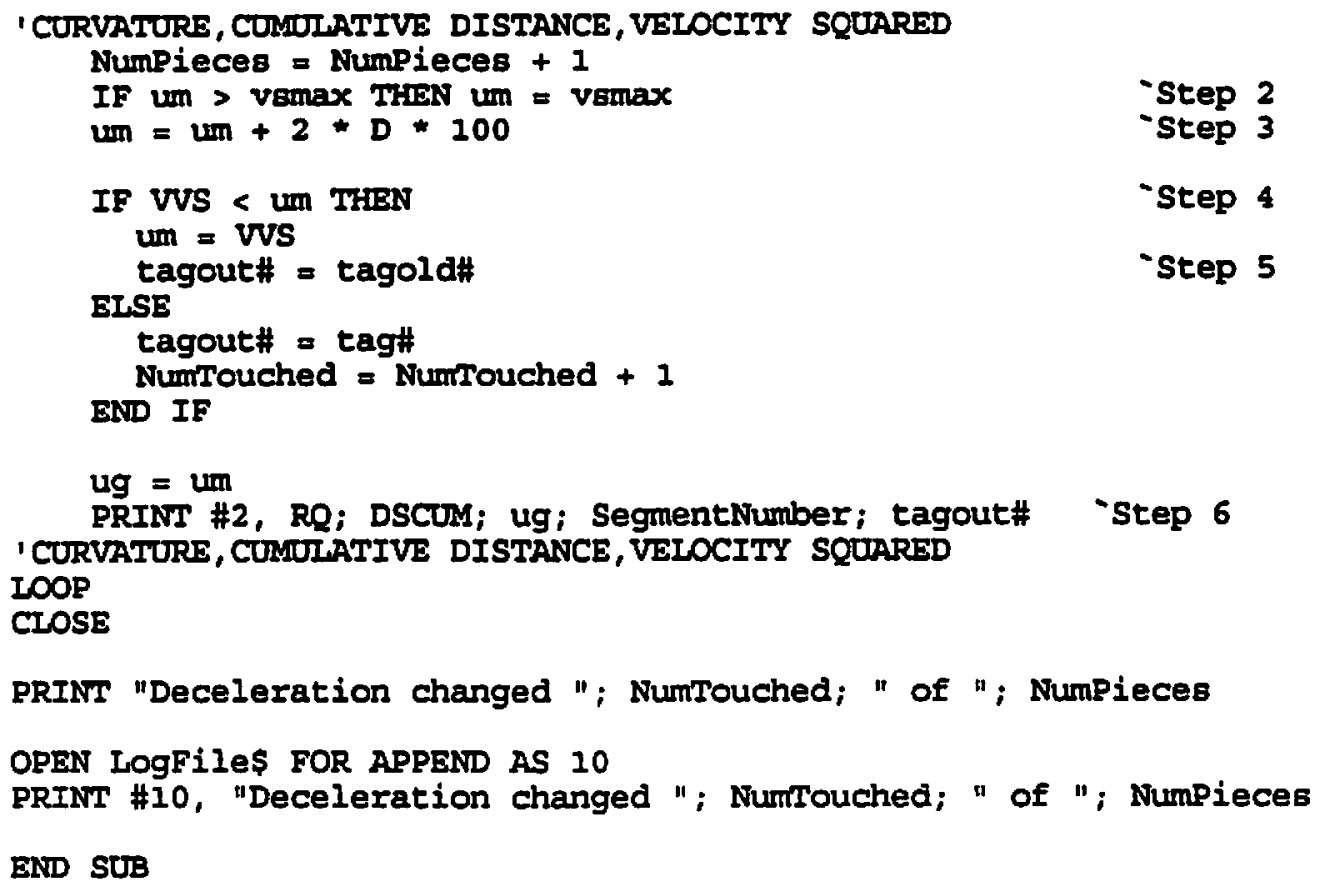

\section{POST PROCESSOR SUBS - Smoothing}

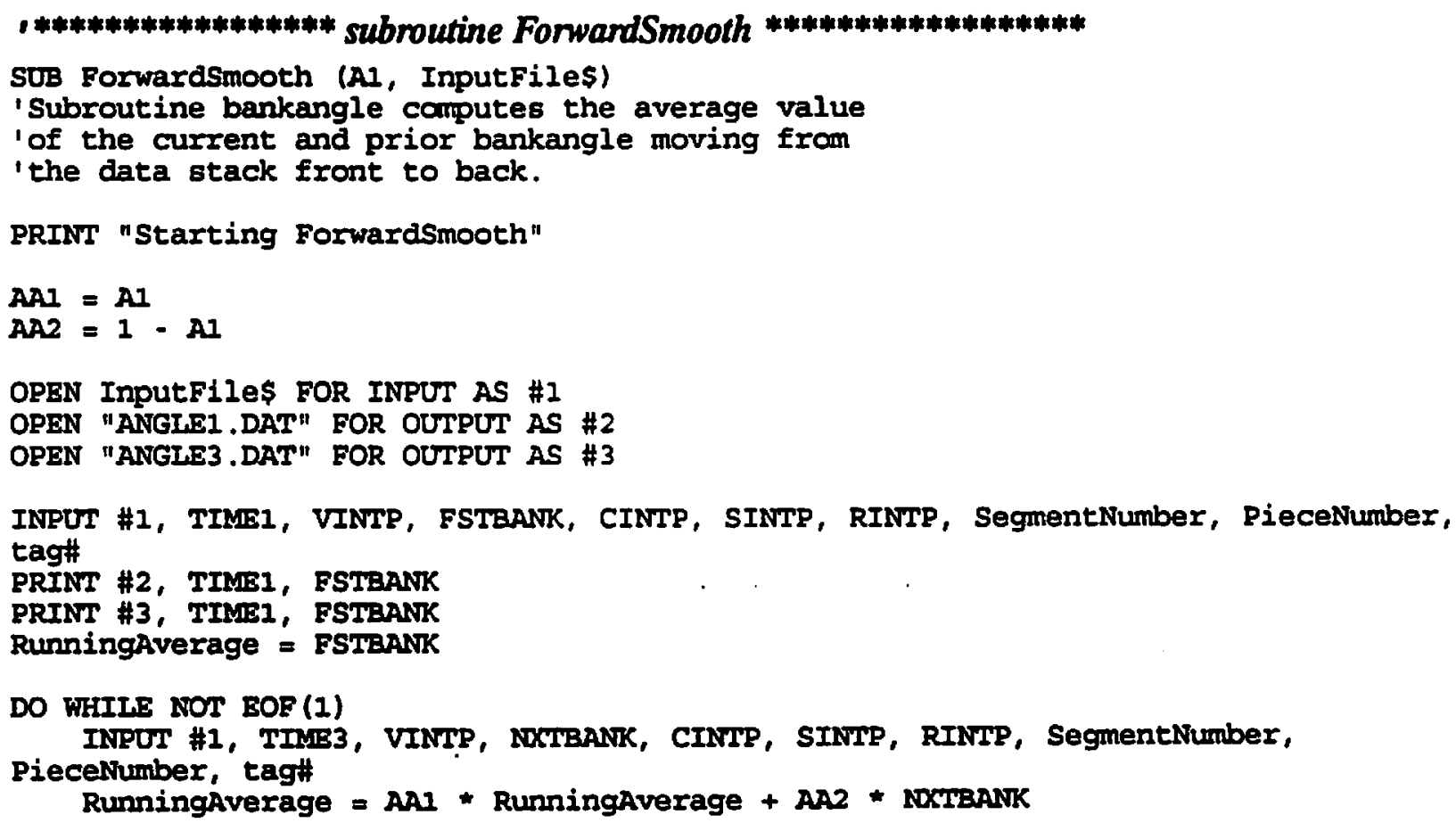




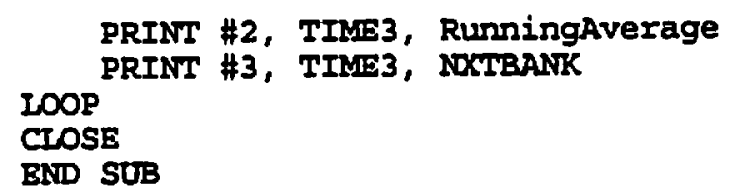




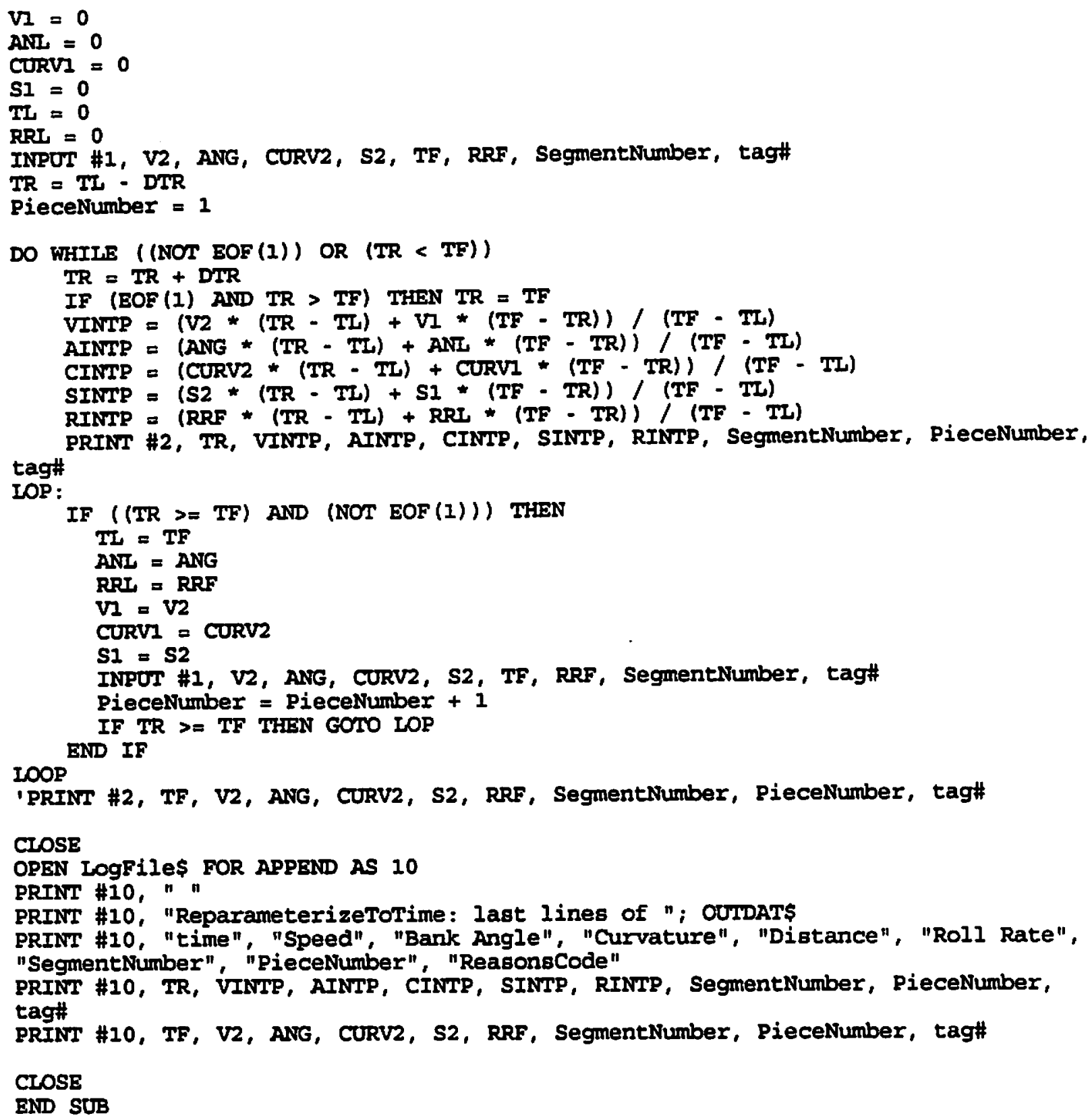


OPEN infiles FOR INPUT AS \#1

OPEN "Indax. tmip" FOR RANDOM AS \#2 IEN = 24

$N=0$

DO WHILE NOT EOF (1)

$\mathbf{N}=\mathbf{N}+\mathbf{1}$

INEOT \#1, datum. sernum, datum.REVERSE, datum.SpeedSquared,

datum. Segmentrumber, datum.tagg\#

IOOP PUT \#2, , datum

CLOSE \#1

OPEN OUEfiles FOR OUTPUT AS \#3

FOR $J=N$ TO 1 STEP - I

GET \#2, J, datum

PRINT \#3, datum.sernum; datum.REVERSE; datum.SpeedSquared;

datum.Segmentrumber; datum.tagg\#

NEXT J

CIOSE \#3

CLOSE \#2

KIII "Irdax. tmp"

END SUB

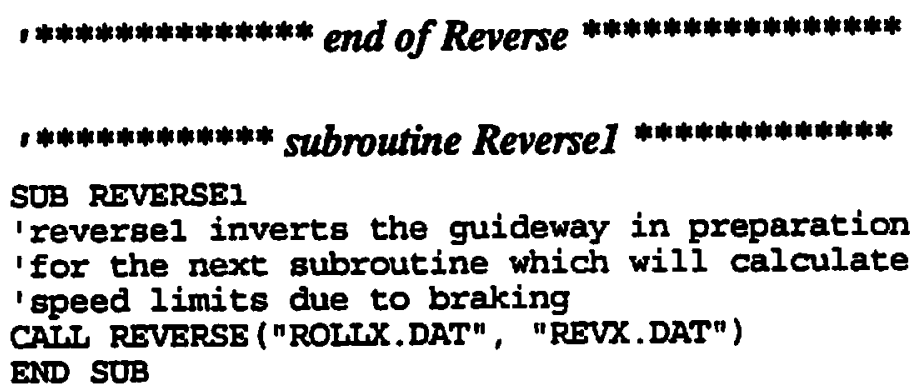




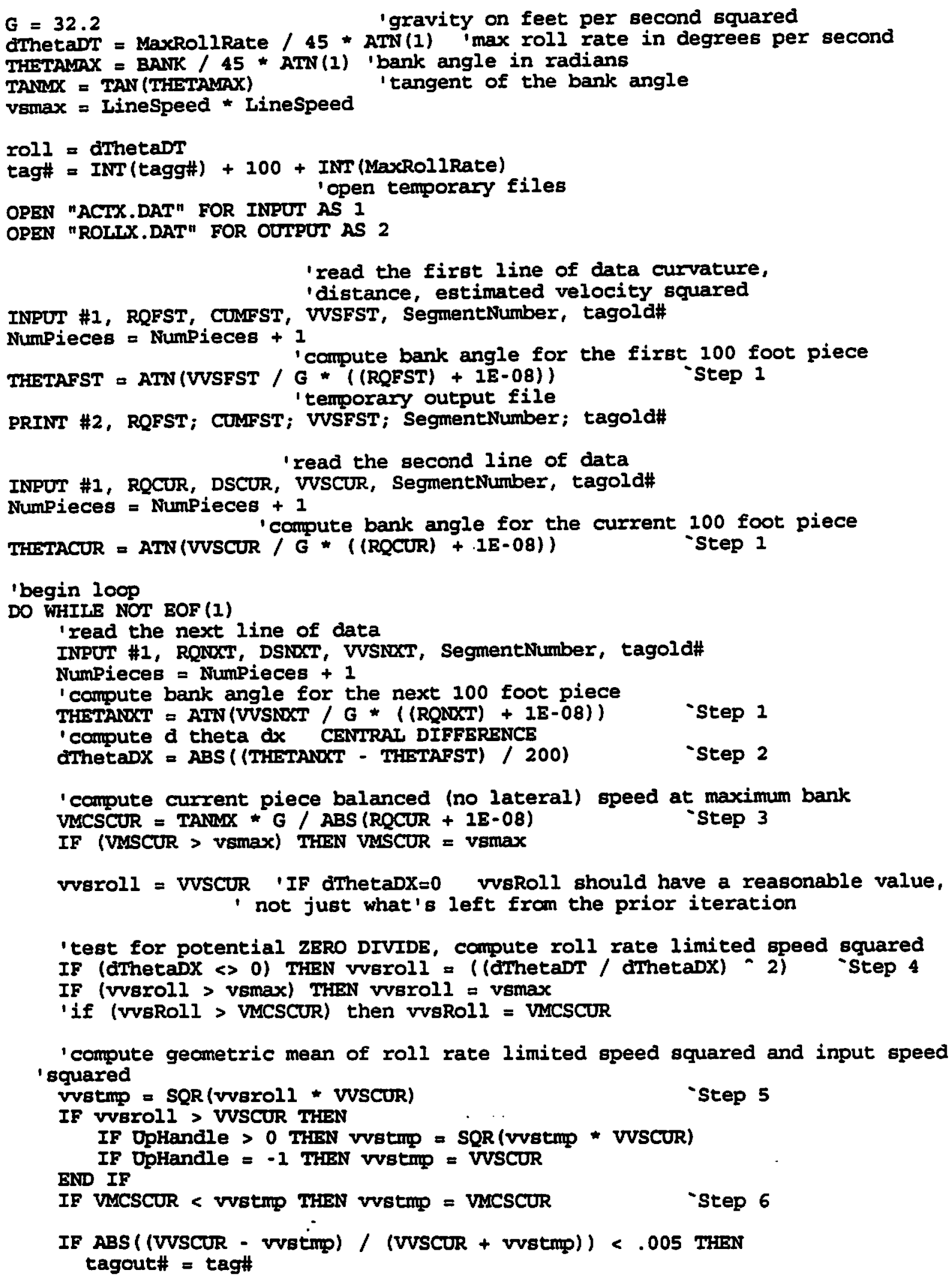




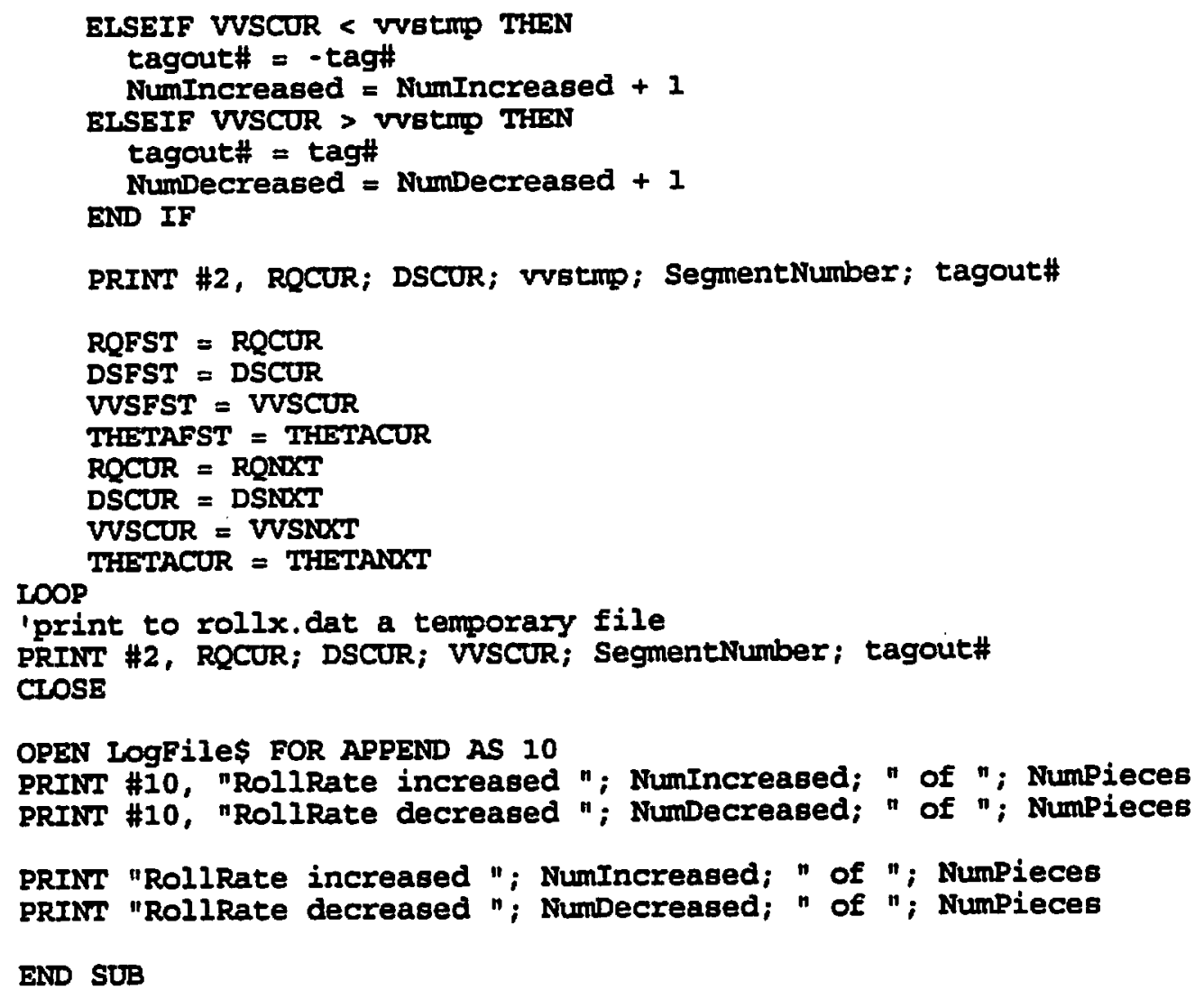


CIOSE \#3

CLOSE \#2

KIIL "Indax.tmp"

END SUB

1*mm****** end of SmoothReverse ***********

1********** subroutine SmoothReverse1

SUB SmoothReversel (InputFiles)

' SmoothReversel inverts the data in preparation

I for the next subroutine which will calculate

'an exponential moving average backwards

CALL SmoothReverge (InputFiles, "FLIPPED.DAT")

BND SUB

1************ end of SmoothReverseI

1***********abroutine SmoothReverse 2

SUB SmoothReverse2

' SmoothReverse2 inverts the data after

'an exponential moving average backwards

CAIL SmoOthReverge ("ANGLEREV.DAT", "ANGLE2 .DAT")

END SUB

1************ end of SmoothReverse2 


\section{Appendix C. KINCALC.SAS: Brief Description and Program}

KINCALC.SAS is a SAS program which calculates a motion sickness dose value (MSDV) from a sequence of vertical accelerations. The resulting value may be used for comparing two proposed Maglev alignments. Also, the program could be used to locate segments of the trajectory which make large contributions to the MSDV.

The program KINCALC.SAS makes use of a SAS procedure, Proc Spectra, (part of the ETS module) to calculate the periodogram of the vertical accelerations. Other software packages are available for calculating a periodogram.

KNNCALC.SAS applies the $W_{\mathrm{f}}$ filter for quantifying the motion sickness potential of an input sequence of vertical accelerations (see ISO 2631). This is done by applying a weight function to the periodogram of the vertical accelerations. The program could easily be modified to apply other weight functions such as are described in ISO $2631\left(\mathrm{e} . \mathrm{g}\right.$. $\mathrm{W}_{\mathrm{k}}$ ) and could work with accelerations along axes other than the vertical axis.

The program, KNNCALC.SAS, takes as input a file (ACCEL.DAT) which contains a sequence of longitudinal ( $\mathrm{xcg}$ ), lateral (ycg), and vertical (zcg) accelerations measured in hundredths of a $\mathrm{g}$. The sampling rate is 10 measurements per second. The sampling rate should be at least twice as high as the highest frequency considered important. For the $W_{f}$ weight function about 1 or 2 measurements per second is enough, but for other weight functions presented in ISO 2631 this would need to be much higher.

KNNCALC.SAS was written to calculate the motion sickness dose value for the 9 flights of this study. For that application, the accelerations in the input file (ACCEL.DAT) were actually measured using accelerometers. To use KDNCALC.SAS on the output of COMBO.BAS (the *.TIM file - see Appendices $A$, and $B$ ), in addition to renaming the *.TIM file as ACCEL.DAT, an additional calculation is necessary. Note that the ${ }^{*}$.TIM file contains a sequence of bank angles instead of a sequence of vertical acceleration. By assuming all accelerations to be resolved through the vertical axis (with respect to the passenger) the acceleration (in $\mathrm{g}$ ) experienced by a passenger traversing a curve must be calculated using the formula

$$
z g=\frac{J}{\cos (b a n k)}-I
$$

This calculation is presented in KINCALC.SAS in the second "data step" (commented out) which should be used place of the first data step. The replacement data step is shown below.

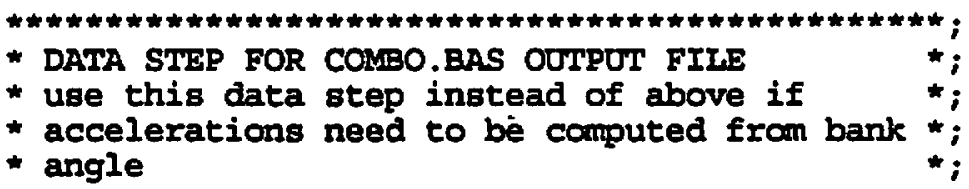


* data taeries;

* infile 'accel.dat';

* INPUT bank;

* $\quad 2 g=1 / \cos (b a n k \star \varepsilon P I / 180)-1$;

* z=zg*9.8; $\quad$ * Convert to $\mathrm{m} / \mathrm{s} \mathrm{s}^{-2} \hbar_{j}$

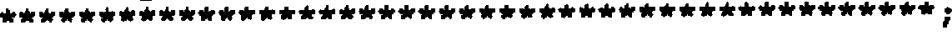


Annotated Code: KINCALC.SAS

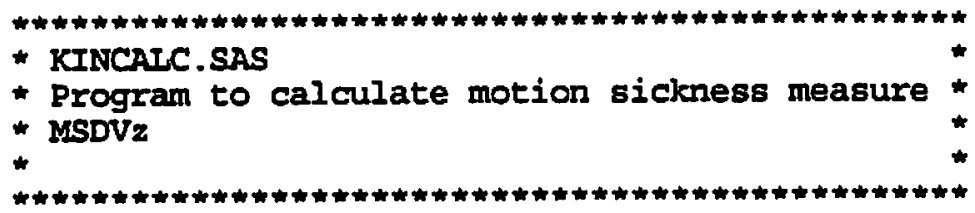

YIET SAMPRATE $=10$; Adjust if other rate is used;

VIET $P I=3.141592654$;

data tseries;

infile 'accel.dat' ;

INPOT xcg ycg zcg;

$\mathrm{z}=\mathrm{zcg} / 100 * 9.8$; $\quad$ Convert to $\mathrm{m} / \mathrm{s}^{-2}$ *; $^{\circ}$

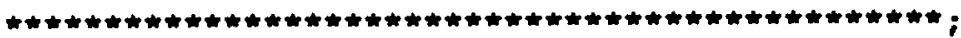

- DATA STEP FOR COMBo.BAS OUTPUT FIIE *;

- use this data step instead of above if *;

- accelerations need to be computed from bank *;

* angle

* data tвeries:

- infile 'accel.dat' ;

- INPUT bank;

- $\quad \mathrm{zg}=1 / \mathrm{cos}(\mathrm{bank} \star 6 \mathrm{PI} / 180)-1$;

* z=zg*9.8; $\quad$ Convert to $\mathrm{m} / \mathrm{s}^{\wedge} 2$;

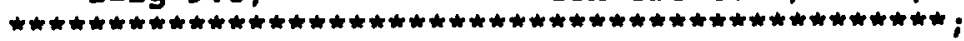

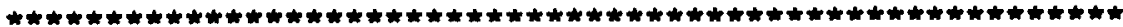

* Proc spectra converts the acceleration sequence into *

* the periodogram. The output of this proc

* (contained in a data set "spec_out" is:

* freg - frequency in radians per unit time (tenths of *

* a second)

* p_01 - the value of the periodogram at the given *

* frequency.

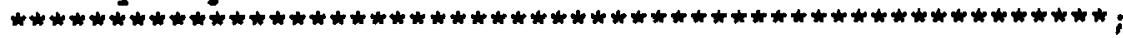

proc spectra data=tseries out=spec_out ;

var z;

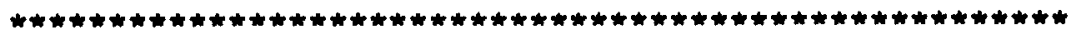

* squarit (equare it) is a macro for obtaining the *

* squared modulus of a quadratic in $z=i f$ where $i$ is *

- the square root of -1 and $f$ is an input Erequency *

- in CQB.

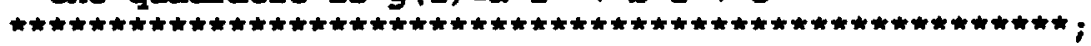

smacro squarit;

$f=2 \star \& P I \star f h z ; f f=f \star f ;$

$g=a \star a \star f f \star f f+(b \star b-2 * c \star a) \star f f+c \star c ;$

imend squarit;

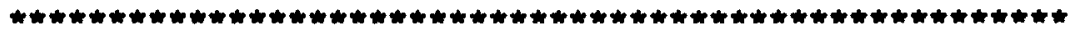

* The data step "filter" calculates the weighting *

* function and the sum of the weighted periodogram *

* The filter is specified.by constants $\mathrm{f1}$.. $\mathrm{f} 6$ and *

- q4 - 96

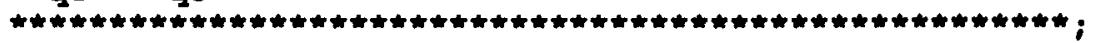




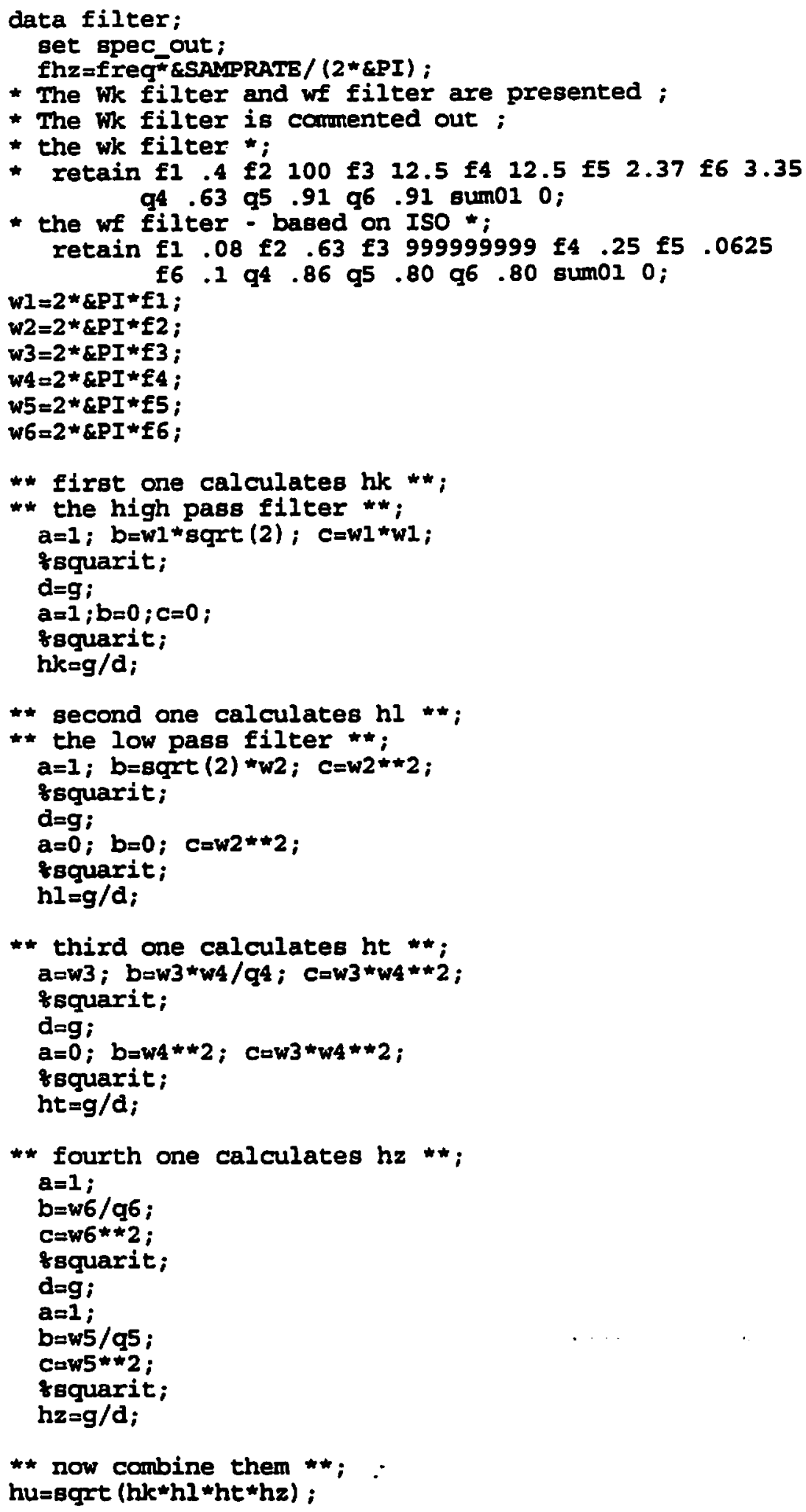




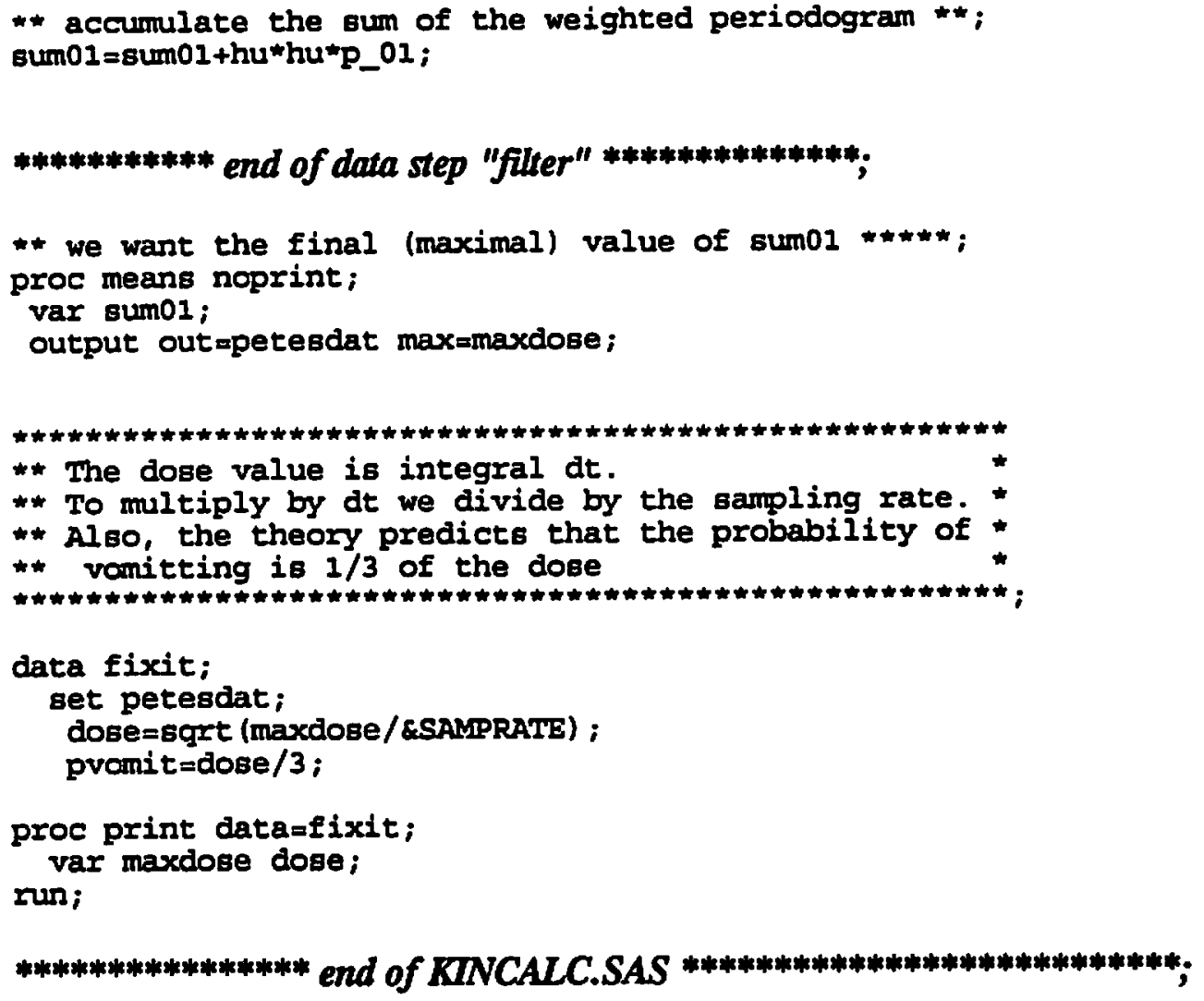




\section{Appendix D: ALIGNMENT.BAS and New York State Data}

\section{PROGRAM LOGIC}

The purpose of the program ALIGNMENT.BAS is to transform engineering data describing a proposed Maglev alignment along sections of the New York State Thruway into the form required by the program COMBO.BAS. ALIGNMENT.BAS is a BASIC program which uses interpolation to reconstitute the New York State guideway horizontal geometry with spirals. Spirals are computed subject to: maneuvering distance, target radius of curvature, and a spiral length limit of 1000 feet. $^{2}$ Spiral type could be anything; linear spirals are presently implemented. Where segment length is less than 2000 feet, target segment curvature will not achieved. Current implementation does not conserve change in heading. Conservation of change of heading can easily be implemented when appropriate.

Spirals are computed as linear rather than the sinusoidal shape used in the New York design because the specific rate of change of the bank angle for flying passengers is controlled by the aircraft pilot and because linear spirals were considered appropriate to the ride quality mission.

Input is a batch file containing segment radius and length. Standard segment data units are feet for horizontal data.

Output is to a disk file, RECONST.ROE, and has curvature and cumulative distance every 100 feet, and the segment number.

\section{SPECIFIC MODELING LOGIC}

The step numbers refer to the steps of logic and correspond to lines in the annotated code directly following this section.

Step 1) Compute curvature for each segment using the input radius. The resulting value is the given curvature somewhere within the segment.

Step 2) Use interpolation to compute a boundary curvature between each pair of adjacent segments.

Step 3) Divide each segment into very small pieces (100 feet each)

Step 4) Compute distance $X$ from the segment boundary to the current piece being computed.

Step 5) Normalize distance $\mathrm{XN}$ between the current point and the point at which it could be at maximum curvature.

\footnotetext{
${ }^{2}$ Maximum spiral length is set to 1000 feet.
} 
Step 6) Compute current point curvature using linear interpolation between beginning boundary curvature and the point at which it could be at maximum segment curvature, moving forward.

Step 7) Compute piece curvature using linear interpolation between end boundary curvature and to the segment curvature, moving backward.

NOTE: Since the initial boundary curvature and the final boundary curvature are not necessarily equal, the slopes and the lengths of the two spirals are independent of one another. Spiral shape is implemented as linear but can be altered, for example, to clothoid or sinusoid.

Step 8) If the sum of the spiral lengths is equal to total segment length, there is no constant curvature section. If the sum of the spiral lengths is less than total segment length, there is a constant curvature section.

Step 9) Output results are curvature every 100 feet and cumulative distance in feet.

\section{'ALIGNMENT.BAS ANNOTATED CODE}

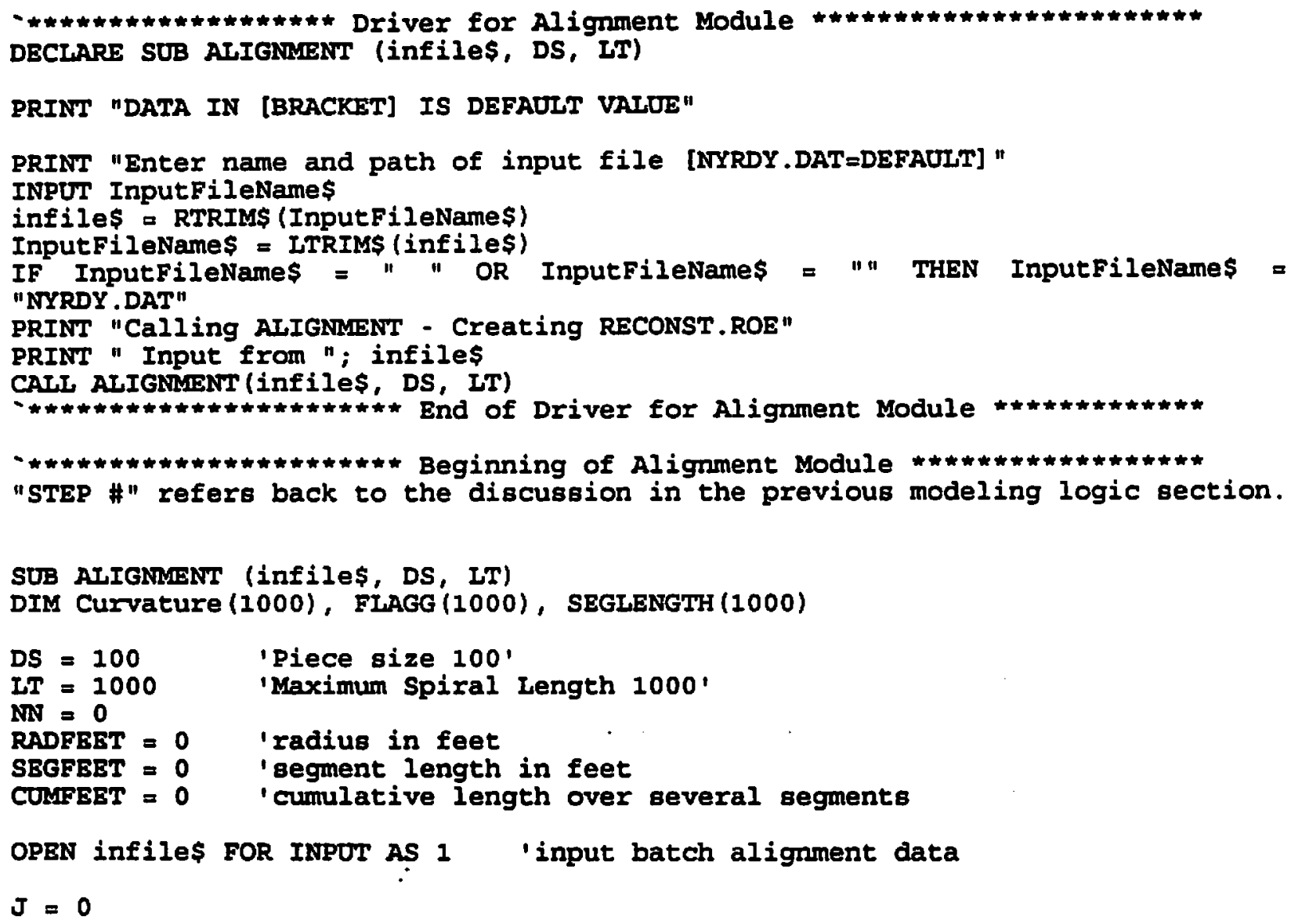




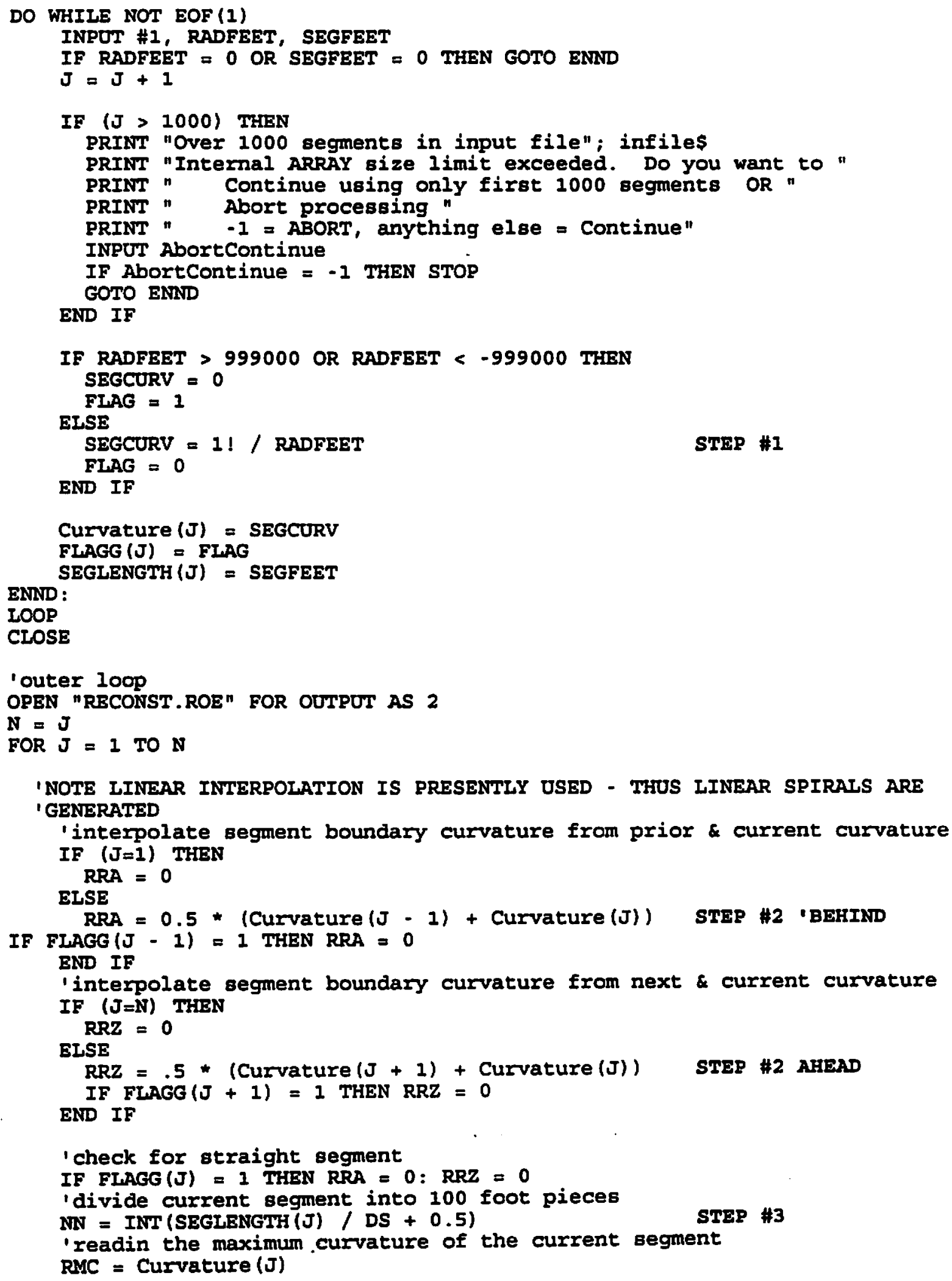


'begin JJ inner loop calculation for each piece in current segment FOR $J J=1$ TO NN

' working from the beginning of the segment forward

$\mathrm{X}=\mathrm{JJ} \star \mathrm{DS}$

$X \mathrm{XN}=\mathrm{X} / \mathrm{LT}$

STEP \#4 AHEAD

STEP \#5

'NOTE LINEAR INTERPOLATION IS PRESENTLY USED - THOS IINEAR SPIRAIS ARE

' GENERATED

'interpolate between piece beginning boundary curvature and maximum curvature

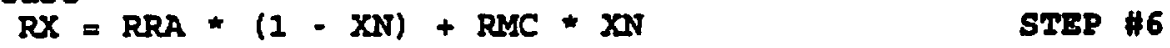

'working from the end of the segment backwards

$Y=\operatorname{SEGLENGTH}(\mathrm{J}) \cdot \mathrm{X}$

$\mathrm{YN}=\mathrm{Y} / \mathrm{IT}$

STEP \#4 BEHIND

STEP \#5

'NOTE LINEAR INTERPOLATION IS PRESENTLY USED - THUS LINEAR SPIRALS ARE

' GENERATED

interpolate between piece ending boundary curvature and maximum curvature

$R Y=R R Z *(1 \cdot Y N)+R M C * Y N$

STEP \#6

$R Q=R M C$

IF JS $\Leftrightarrow=10$ AND JJ $\Leftrightarrow$ NN $/ 2$ THEN RQ $=R X$

IF JJ > NN / 2 AND JJ > NN -10 THEN RQ $=$ RY

IF JJ $<=((L T+1) / D S)$ AND JJ $s=$ NN $/ 2$ THEN RQ $=R X$

IF JJ > NN / 2 AND JJ > NN - $((I T+1) /$ DS $)$ THEN RQ = RY

- test for straight track and adjust curvature

IF RMC $=0$ THEN RQ $=0$

STEP \#日

COMFEET $=$ COMFEET +100

PRINT \#2, RQ, CUMFEET, J

STEP \#9 NEXT JJ

NEXT J

CLOSE

END SUB 
The New York State Data (NYRDY.DAT) follows: (Column 1 is radius; column 2 is curve length) - read down, then across. Data is taken directly from Ref. 7.

\begin{tabular}{|c|c|c|c|c|c|c|c|c|c|c|c|}
\hline radius & length & radius & length & radius & length & radius & length & radius & length & radius & length \\
\hline 999999 & 7250 & 19100 & 4510 & 999999 & 520 & 999999 & 8305 & -30000 & 690 & 999999 & 1995 \\
\hline 6800 & 4000 & 999999 & 1795 & -13300 & 1870 & -16000 & 2955 & 999999 & 9720 & -10000 & \\
\hline 999999 & 3780 & -19100 & 50 & 999999 & 2955 & 999999 & 4140 & 30000 & 4110 & -15000 & 62 \\
\hline 19800 & 1445 & 19100 & 3215 & 25000 & 2135 & 13300 & 2780 & 9000 & 3620 & 999999 & 355 \\
\hline 999999 & 2275 & 999999 & 1430 & 999999 & 3990 & 999999 & 1240 & 999999 & 800 & 80000 & 8 \\
\hline-12000 & 4195 & 12000 & 4330 & 999999 & 620 & 14000 & 2480 & -10000 & 4665 & 999999 & \\
\hline 999999 & 2010 & 999999 & 635 & -15000 & 2605 & 999999 & 6640 & -19100 & 4450 & -2800 & 312 \\
\hline 32000 & 1015 & -12000 & 4225 & 999999 & 790 & -14000 & 2415 & 999999 & 1295 & 999999 & 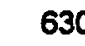 \\
\hline 999999 & 1395 & 12000 & 2605 & 15000 & 1420 & 999999 & 4760 & 11000 & 4860 & 3800 & 512 \\
\hline-19100 & 4410 & 999999 & 2020 & 999999 & 6980 & 12000 & 2315 & 30000 & 3815 & 999999 & 28 \\
\hline 13300 & 3715 & 2800 & 2520 & 19100 & 1505 & 999999 & 3250 & 999999 & 1360 & -3200 & 459 \\
\hline-19100 & 2390 & 999999 & 455 & 999999 & 2325 & 30000 & 685 & -19100 & 4600 & 999999 & 6 \\
\hline 999999 & 830 & -10000 & 2160 & -19100 & 1305 & 999999 & 8940 & 999999 & 8705 & 2800 & 38 \\
\hline 28000 & 2975 & 999999 & 1675 & 999999 & 1160 & 90000 & 1245 & -20000 & 4355 & 999999 & 6 \\
\hline-13300 & 3400 & -4200 & 3375 & -15000 & 5075 & 999999 & 6140 & 20000 & 2545 & 2800 & 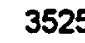 \\
\hline 6000 & 3635 & 999999 & 3980 & 999999 & 2160 & -20000 & 1510 & 999999 & 645 & 999999 & 6 \\
\hline 999999 & 5010 & 20000 & 1220 & 10000 & 4625 & 999999 & 9595 & -20000 & 5050 & .5500 & 289 \\
\hline-10000 & 1845 & 999999 & 1230 & 999999 & 330 & -19100 & 2285 & 999999 & 4850 & 999999 & \\
\hline 399999 & 1720 & 6000 & 4140 & -19100 & 2315 & 999999 & 12550 & 13300 & 2485 & 12000 & 10 \\
\hline 19100 & 3220 & 999999 & 2825 & 999999 & 3330 & 999999 & 735 & 999999 & 855 & & \\
\hline 999999 & 1420 & 13300 & 4055 & -8000 & 2100 & 30000 & 800 & -19100 & 3195 & & \\
\hline-8000 & 1890 & 999999 & 2120 & 999999 & 725 & 999999 & 3985 & 999999 & 7065 & & \\
\hline 999999 & 1570 & -4000 & 2985 & 8400 & 3895 & 11000 & 5280 & 19100 & 3815 & & \\
\hline 8000 & 1495 & 999999 & 855 & 999999 & 3485 & 999999 & 2795 & 999999 & 2895 & & \\
\hline 399999 & 1250 & 20000 & 1705 & -4200 & 4485 & -11000 & 5760 & 19100 & 3340 & & \\
\hline-10000 & 2890 & 999999 & 1580 & 999999 & 5475 & 80000 & 3855 & 999999 & 2670 & & \\
\hline 999999 & 1015 & 20000 & 1450 & 9000 & 4235 & 999999 & 490 & 19100 & 5805 & & \\
\hline 10000 & 2880 & 999999 & 3980 & 999999 & 8895 & -9000 & 3155 & -19100 & 6180 & & \\
\hline 999999 & 225 & -13300 & 2710 & -19100 & 4905 & 999999 & 2595 & 999999 & 6785 & & \\
\hline-11000 & 2715 & 20000 & 2470 & 999999 & 2625 & -30000 & 640 & -15000 & 6675 & & \\
\hline 999999 & 1505 & 999999 & 3770 & -13300 & 5615 & 999999 & 7630 & 999999 & 5850 & & \\
\hline 6000 & 3135 & -19100 & 2515 & 999999 & 6380 & 100000 & 7625 & 15000 & 6365 & & \\
\hline-6000 & 3735 & 999999 & 1720 & -19100 & 2340 & 999999 & 1935 & 999999 & 18185 & & \\
\hline 5500 & 5585 & 4400 & 1795 & 999999 & 2585 & 20000 & 4185 & -19100 & 5155 & & \\
\hline-10000 & 2325 & 999999 & 940 & 13300 & 3035 & 999999 & 585 & 19100 & 3445 & & \\
\hline 399999 & 1605 & -7000 & 2115 & 999999 & 7200 & -10000 & 2735 & 999999 & 2475 & & \\
\hline 13300 & 2615 & 999999 & 9020 & -6000 & 2630 & 999999 & 305 & -30000 & 855 & & \\
\hline-13300 & 3600 & 20000 & 2305 & 999999 & 23925 & 25000 & 1855 & 999999 & 8450 & & \\
\hline 999999 & 2650 & 999999 & 1355 & 15000 & 4950 & 999999 & 600 & 19000 & 3700 & & \\
\hline 11000 & 4015 & -25000 & 4700 & 999999 & 2220 & -25000 & 1550 & 999999 & 1940 & & \\
\hline 999999 & 1715 & -1330 & 1055 & -7000 & 3930 & 999999 & 7015 & -19000 & 13320 & & \\
\hline 9200 & 2425 & 999999 & 270 & 999999 & 15050 & -25000 & 1390 & 19000 & 3325 & & \\
\hline 999999 & 3255 & 7000 & 3315 & 14000 & 3035 & 999999 & 3825 & 9000 & 10385 & & \\
\hline-20000 & 3260 & 999999 & 3810 & 999999 & 3850 & 20000 & 4630 & 999999 & 760 & & \\
\hline 999999 & 1810 & -6000 & 2635 & 50000 & 2605 & 999999 & 12320 & -3800 & 4015 & & \\
\hline-8000 & 2305 & 999999 & 1655 & 999999 & 5305 & 30000 & 550 & 999999 & 670 & & \\
\hline 999999 & 600 & 20000 & 640 & -7000 & 2885 & 999999 & 3310 & 5500 & 5445 & & \\
\hline 13 & 1560 & 999999 & 680 & 999999 & $3510^{\circ}$ & $-20000^{\circ}$ & 4540 & 999999 & 1920 & & \\
\hline & 1480 & -20000 & 825 & 9000 & 2935 & 999999 & 1295 & -5000 & 3150 & & \\
\hline & 3245 & 999999 & 2160 & 18000 & 745 & 30000 & 700 & 999999 . & 530 & & \\
\hline & 1730 & 9000 & 2710 & 8000 & 2905 & 999999 & 1960 & 3600 & 3800 & & \\
\hline
\end{tabular}


Appendix E: Plot of bank angle and roll rate vs. time for the worst case $\left(28^{\circ}\right.$ and $\left.8^{\circ} / \mathrm{sec}\right)$

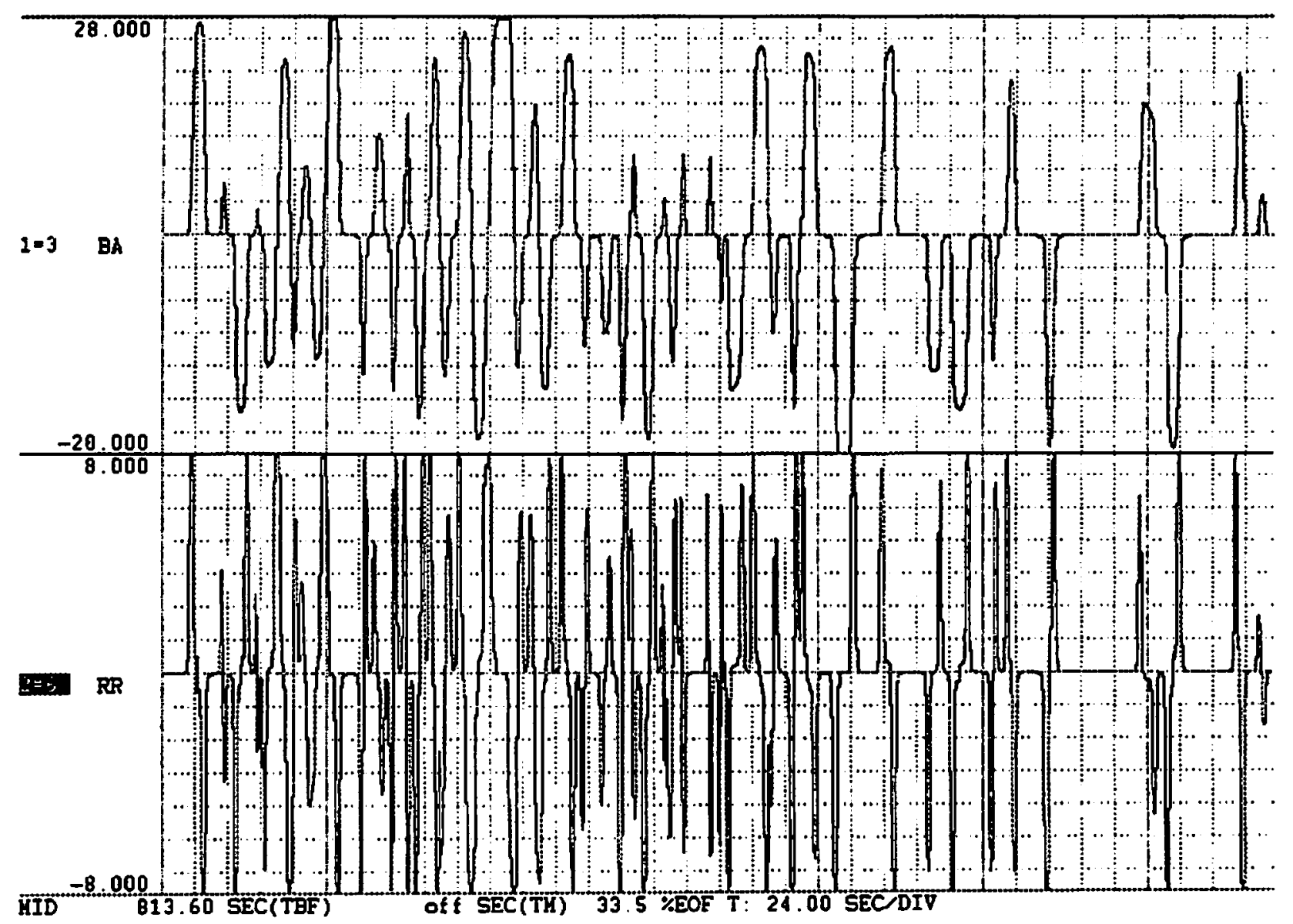




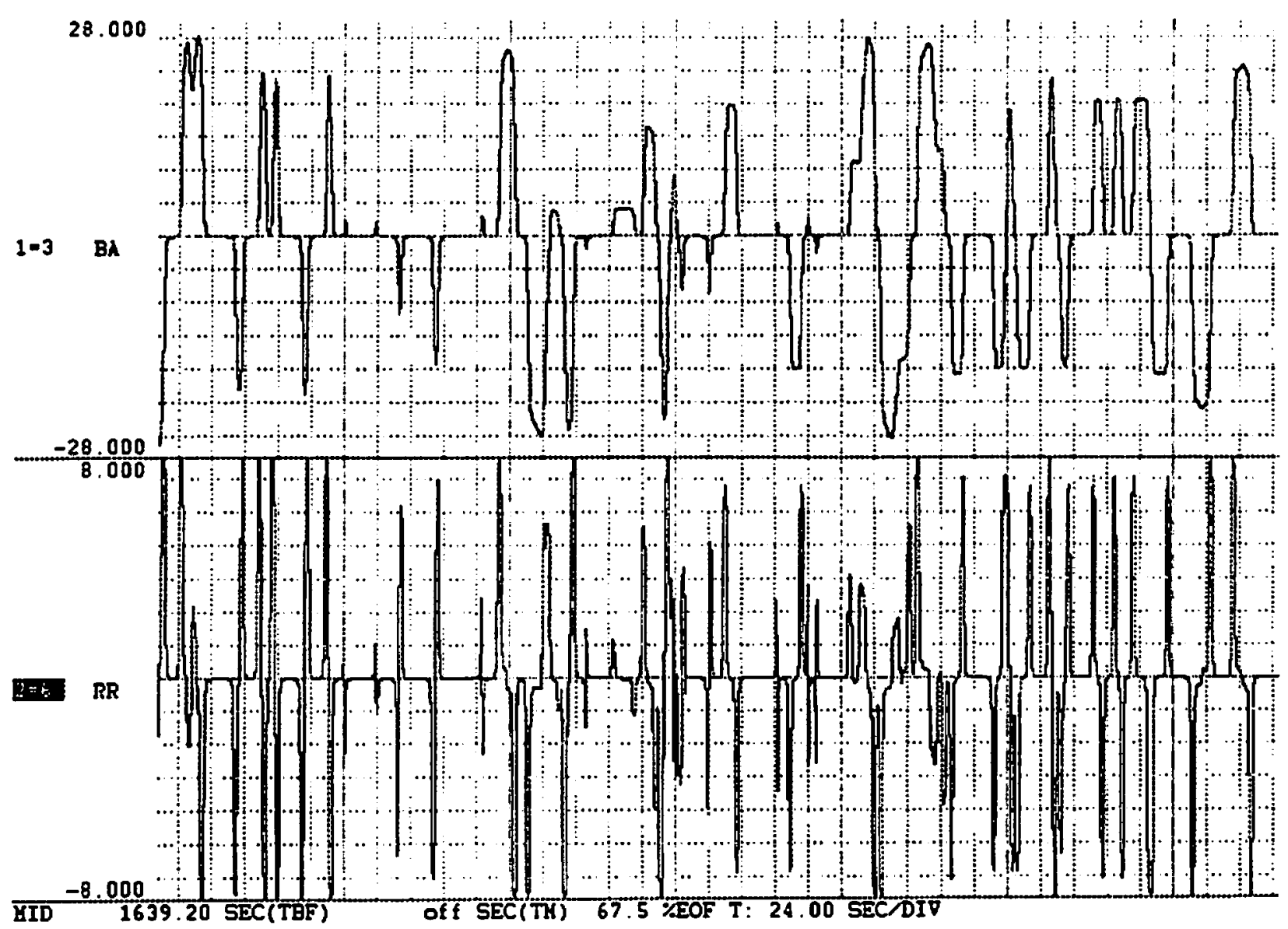




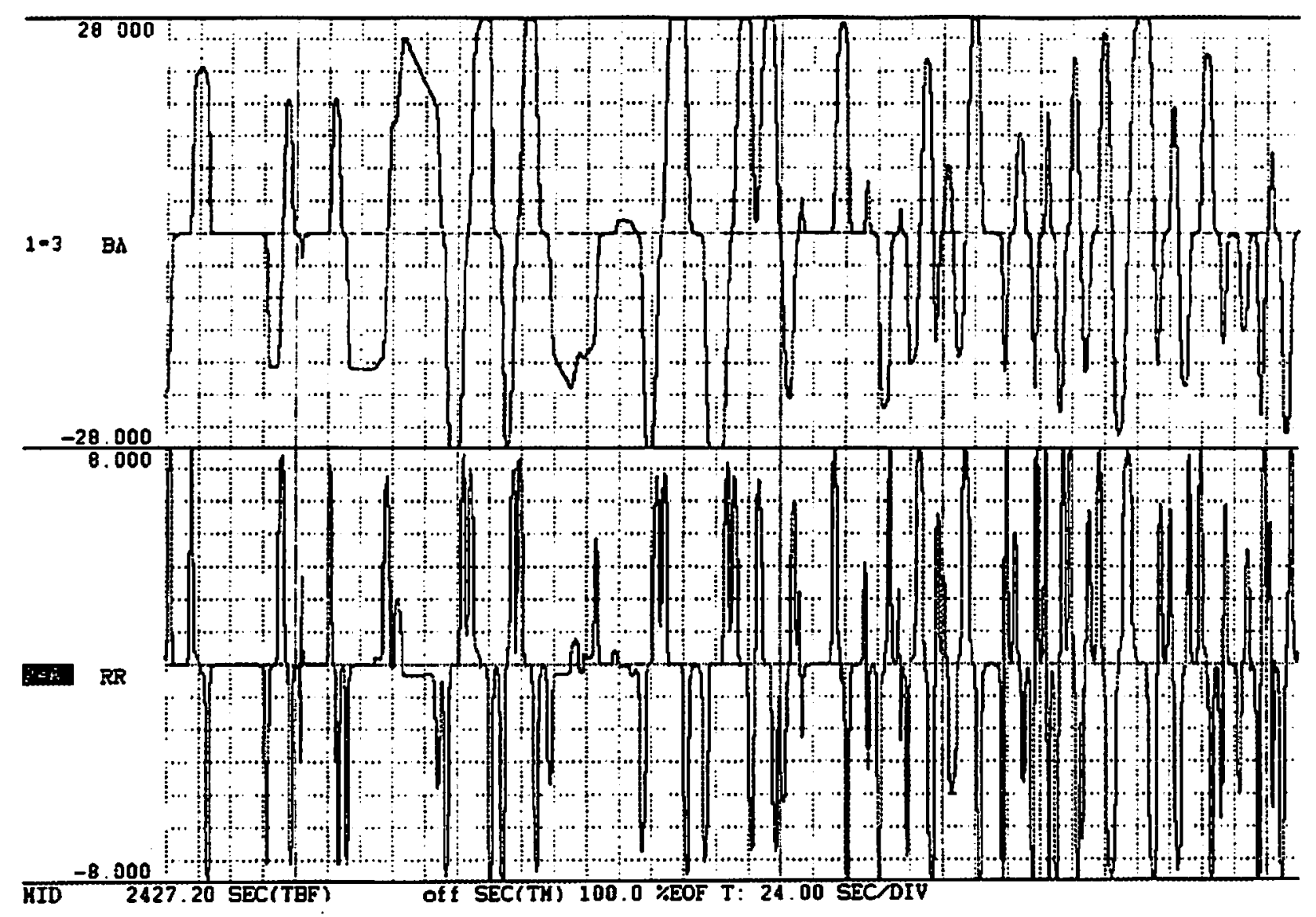




\title{
Appendix F. Subject Consent Form
}

\author{
SUBJECT CONSENT FORM \\ MAGLEV RIDE-QUALITY STUDY
}

$\mathrm{I}$, consent to be a subject in the research project described below.

1. The purpose of this experiment is to help set the design standards for the speed of future high-speed ground transportation systems. Congress has proposed that $300 \mathrm{MPH}$, magnetically levitated (Maglev) systems be demonstrated in this country and that they use existing rights-of-way as much as possible. Since the Maglev vehicles would operate at speeds much higher than conventional trains, their passengers would experience much higher levels of acceleration (also known as g-forces) both vertically and longitudinally, as well as much higher roll rates.

In setting the standards for future systems, it is very important to know what levels of g-forces and roll rates are acceptable to most people. If the allowable levels are set too high in the design standards, many people may refuse to use the system because of the discomfort they experience; if they are set $100 \mathrm{low}$, the system will be more expensive to build and/or will operate at a lower average speed. The goal of this experiment is to determine the point at which passengers would just begin to experience motion sickness.

2. I have been selected to participate in this study as a representative member of the traveling public, who has made at least six round trips by air, of which at least two occurred in the past year.

3. I understand that in the experimental session I will be flown in a 20-passenger twin turboprop aircraft for about two hours total, of which 45 minutes to one hour will consist of roll maneuvers simulating a Maglev train following the portions of the right of way of the New York State Thruway. These roll manetrvers may involve bank angles as high as 28 degrees, which are stightly higher than the maximum bank angles ordinarily used by commercial airliners (25 degrees). The vertical maneuvers may produce accelerations of as much as $0.2 \mathrm{~g}$ greater than normal. (For comparison, accelerations experienced in typical elevators are about .15 g.) Maneuvers may occur as frequently as four or five per minute. I understand that the risk of injury involved in this experiment is similar to that of flying in a commercial airliner.

4. I understand that in filling out my rating booklet, I will disclose my age, sex and occupation along with my ratings for ride comfort and whether I am experiencing any degree of motion sickness. My name will not be recorded in the subject booklet or in any other experimental records, except this

Page 1 of 2 
consent form and the receipt for the fee. I understand that all reasonable efforts will be made to keep my identity. confidential.

5. I understand that I may contact the following individual with any questions I may have about this study or my participation in it as a research subject:

John K. Pollard, Project Manager

U. S. Dept. of Transportation. DTS-45

55 Broadway

Cambridge. MA 02142

(617) 494-2449

6. I understand that in the unlikely event of a physical injury, emergency care will be provided.

7. I understand that certain medical conditions, such as, pregnancy, retinal detachment, back injuries, heart ailments, unusual tendency to motion sickness etc., may be aggravated by greater than normal g-forces. To the best of my knowledge, I do not have any medical or psychological condition that would interfere with my ability to complete my participation in a safe and satisfactory manner. I agree to answer questions regarding my medical condition to insure that no such problems exist.

7. I understand that I may experience some queasiness or the beginnings of nausea in this experiment. I understand that I am free to withdraw from the experiment if I so chose. I understand that the experimental portion of the flight will be terminated if any passenger becomes nauseous.

8. I understand that the flight session will require about two hours of my time and that I will receive compensation of $\$ 50.00$. I understand that if I also take the one-hour simulator ride, I will be paid an additional \$2S.

I have read and understand the various aspects of my participation in this study, all my questions have been answered and I voluntarily agree to participate.

Name:

(Subject, Please print)

Signanure:

Date:

Page 2 of 2 